\title{
Density Functional Theory Studies on the Relative Reactivity of Chloroethenes on Zerovalent Iron
}

\author{
by \\ Dong-Hee Lim \\ A dissertation submitted in partial fulfillment \\ of the requirements for the degree of \\ Doctor of Philosophy \\ (Environmental Engineering) \\ in The University of Michigan \\ 2008
}

Doctoral Committee:

Associate Professor Christian M. Lastoskie, Chair

Professor Kim F. Hayes

Professor Margaret S. Wooldridge

Associate Professor Udo Becker

Assistant Professor Angela Violi 
(C) Dong-Hee Lim

All Right Reserved

2008 
To my family 


\section{Acknowledgements}

I would first like to deeply thank my mother, Cha Soon Park, who supports me in spirit and in finance to study in the U.S. for my Ph.D. Without her love, I could not live and study in the U.S. with my family. My lovely wife, Young Soo Han, studying in the same department in the University of Michigan, has eagerly devoted and scarified herself for me and our family. I have greatly appreciated her help, so I could concentrate on my dissertation research. I also thank my two sons, Hoyeon and Seoyeon (Nathan), who give me the joy of being their father.

At the University of Michigan, I would like to give my special thanks to my advisor, Professor Christian M. Lastoskie, who has supported, encouraged, and advised me on my dissertation research. His warm kindness and sharp intelligence has made me succeed in the long journey of Ph.D life. I would also like to thank all my dissertation committee members. I appreciate that Professor Kim Hayes suggested my very interesting dissertation topic. Together with Professor Avery Demond, they have expressed kind concern for my family and have supported me and Young Soo. I greatly thank Professor Udo Becker for his steadiness in advising me on quantum simulation questions. I also greatly appreciate that Professors Margaret Wooldridge and Angela Violi from the Mechanical Engineering department have shown their warm concern to me and my research, and have guided me through simulation works.

I would like to give my thanks to Professor Jeremy Semrau, Tom Yavaraski and Sung-Woo Lee who had advised me and encouraged me to acquire molecular-level analysis tools. Thanks to my lab friends, Craig Tenney, Hoa Trinh, and Trinh Duc Tran who have always helped me and given me their resources. Thanks also to my Korean friends, who helped me settle in Ann Arbor, shared joys and sorrows together, and gave me advice on my research: Jun Hee Lee, Sung-Phil Hyun, Hoon Young Jeong, Jong-In Han, Young Jae Kang, Dong Joo Kim, Jongwon Lee, Yong-Sub Jung, Hyun-dong Kim, Jeongdae Im, and Sukhwan Yoon. I also thank other friends I met in the department: 
Andrew Henderson, Shui-Chi Chang, Lisa Colosi, Andres Clarens, Tanya Gallegos, and Corrie Clark.

I would also like to gratefully acknowledge the National Science Foundation through TeraGrid resources provided by TACC and SDSC and the Center for Advanced Computing in the University of Michigan for providing computer resources. I also acknowledge Korea Science and Engineering Foundation and the University of Michigan Rackham Graduate School for financial support.

Last but importantly, I would like to thank Pastors Isaac Shin and Sang-Chun Park who led me spiritually to grow. I also give deep thanks to my friends in faith of Jesus Christ who support me with prayer, Sang-Joon Yoo, Tae-Gyun Moon, Yunseok Heo, Juyoung Park, Yoon Jeong Kim and Kevin Nam. Most of all, I return all my glory to God. 


\section{Table of Contents}

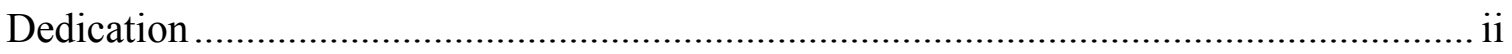

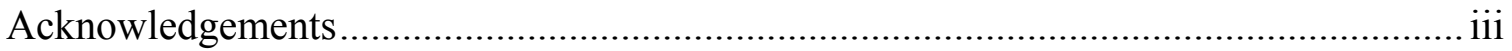

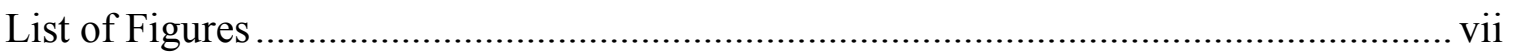

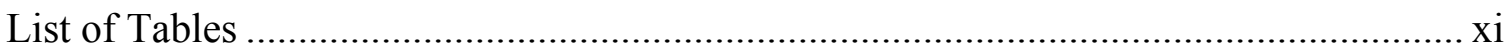

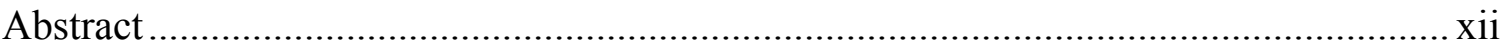

\section{CHAPTER I}

INTRODUCTION AND BACKGROUND.................................................................. 1

1. Introduction..................................................................................................... 1

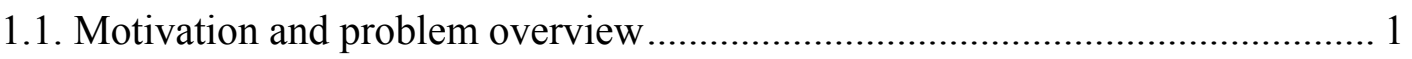

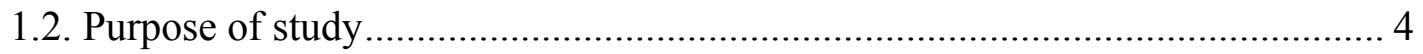

2. Literature Review ................................................................................................................. 5

2.1. Degradation pathways for chloroethenes................................................. 5

2.1.1. Dechlorination mechanisms ............................................................ 5

2.1.2. Applications of abiotic dechlorination................................................ 11

2.2. Adsorption of chloroethenes on metal surfaces ......................................... 12

2.2.1. Di- $\sigma$ - and $\pi$-bond formation.............................................................. 12

2.2.2. Adsorption sites of iron surfaces..................................................... 14

2.2.3. Effect of water molecules ................................................................. 16

2.3. Dissociation of chloroethenes on metal surfaces ........................................... 20

2.3.1. Examples of the investigation of dissociation mechanism .................... 21

2.3.2. Analysis of bond cleavage and formation......................................... 25

2.4. Summary of literature review and chapter overview ................................... 29

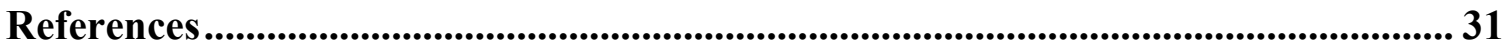

\section{CHAPTER II}

CONSTRUCTION OF IRON SURFACE MODEL ............................................... 36

1. Computational methods .................................................................................... 36

1.1. Density functional theory (DFT) and generalized gradient approximation

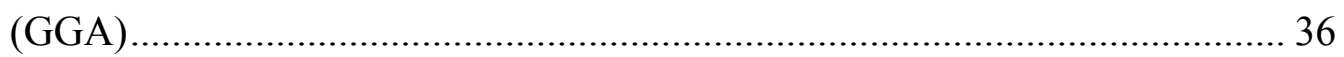

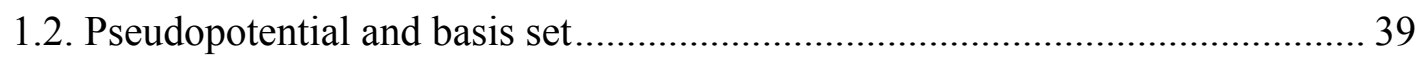

1.3. Cluster model and periodic boundary condition (PBC) model....................... 40

2. Physical properties of bulk iron..................................................................... 42 


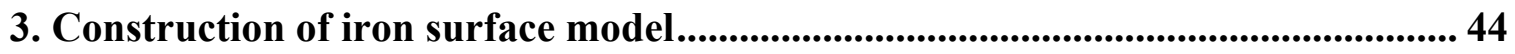

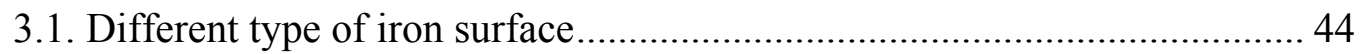

3.2. Periodic three-layer slab model of Fe(110) surface.................................... 46

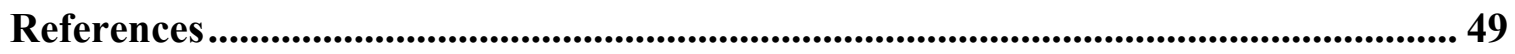

CHAPTER III

ADSORPTION OF CHLOROETHENES ON FE(110)........................................... 51

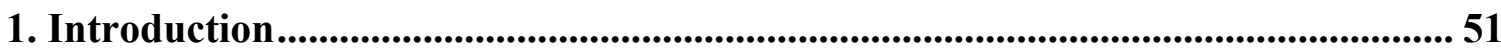

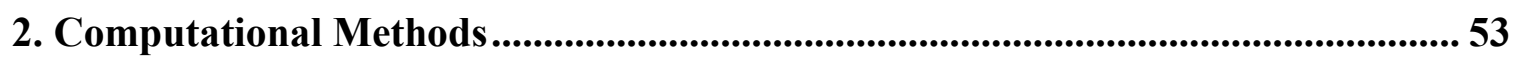

2.1. Adsorption Calculations using Periodic Boundary Conditions........................ 53

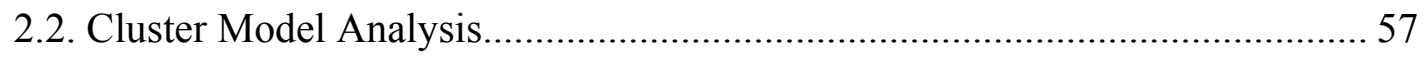

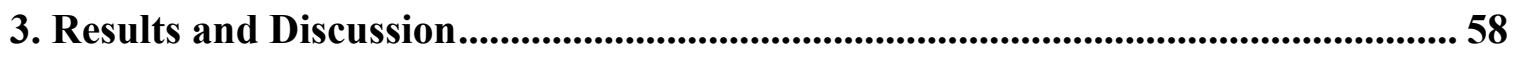

3.1. Effect of supercell size and k-point grid on adsorption ..................................... 58

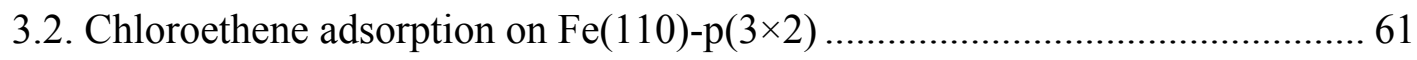

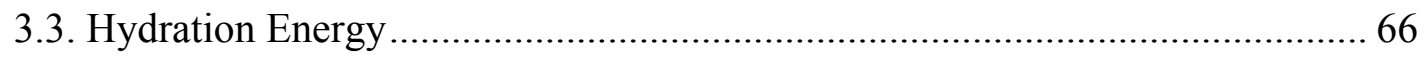

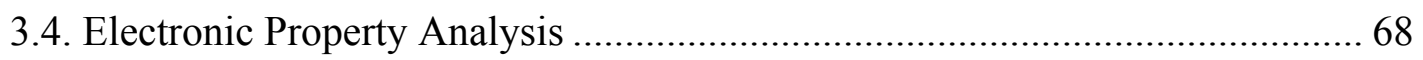

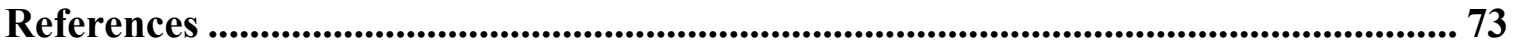

CHAPTER IV

DISSOCIATION OF CHLOROETHENES ON FE(110) ............................................ 76

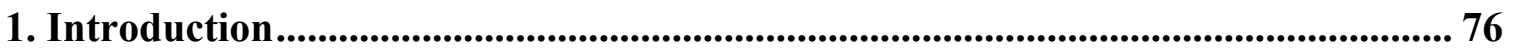

2. Computational Methods.................................................................................................. 77

2.1. Activation energy calculation using climbing image nudged elastic band

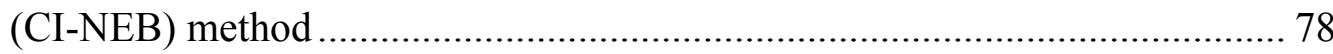

2.2. Reaction rate constant calculation using frequency ........................................ 80

3. Results and Discussion................................................................................................. 82

3.1. Activation energies of PCE, TCE, and cis-DCE on Fe(110) ........................... 82

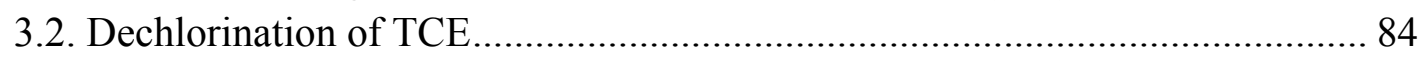

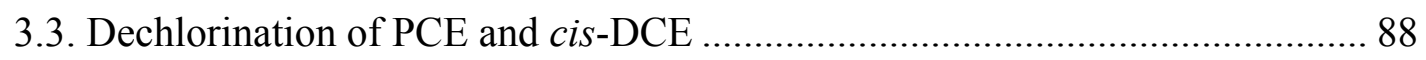

3.4. Dechlorination rate constants of PCE, TCE, and cis-DCE on Fe(110) ........... 92

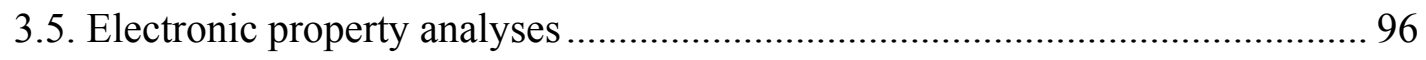

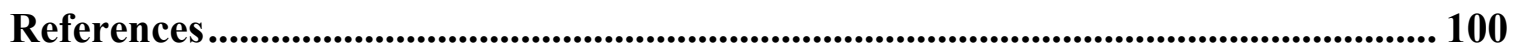

\section{CHAPTER V}

CONCLUSIONS AND FUTURE WORK................................................................ 104

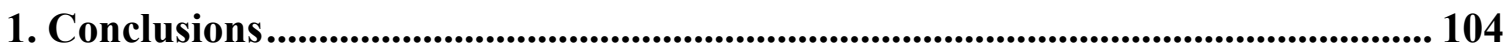

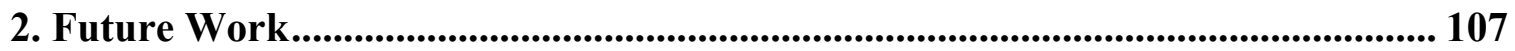




\section{List of Figures}

Figure I-1. Reaction pathways for anaerobic reductive dechlorination of PCE. ...........5

Figure I-2. Reaction pathway for aerobic oxidation of TCE. ......................................... 6

Figure I-3. Hypothesized reaction pathways for abiotic reduction of PCE on zerovalent iron. Numbers correspond to each reaction. Reductive $\beta$ elimination: 2, 6, 8 and 10; hydrogenolysis: $1,3,4,5,7,9,14,17$ and 18 ; a-elimination: 11; hydrogenation: 13,15, 16 and 19. Dotted circles indicate the kinetically-favored pathway for reduction of each product. Percentages represent the amount of product transformed through the pathway...................................................................................................... 8

Figure I-4. Reaction pathways for reductive dechlorination in anoxic aqueous system. 9

Figure I-5. Hypothesized mechanisms for dechlorination of PCE. Reductive $\beta$ elimination may proceed via steps $1,2,3 \mathrm{a}$ and $4 \mathrm{a}$ while hydrogenolysis proceeds via steps $1,2,3 \mathrm{~b}$ and $4 \mathrm{~b}$.

Figure I-6. High-symmetry adsorption sites on $\mathrm{Fe}(110)$ (a) and $\mathrm{Fe}(100)$ (b) surfaces. OT: on-top position; LB: long-bridge; SB: short-bridge; TF: three-fold; H: hollow; B: bridge. Surface $(1 \times 1)$ cells for both $\mathrm{Fe}(110)$ and $\mathrm{Fe}(100)$ are also shown................................................................................................ 14

Figure I-7. Potential reaction pathways for methanol oxidation on $\operatorname{Pt}(111)$ in the vapor and the aqueous phases. The solvated products for each step include an $\mathrm{H}_{3} \mathrm{O}^{+}{ }_{\text {(aq) }}$ group nearby in solution 18

Figure I-8. Dechlorination reaction path for the di- $\sigma$-bonded TCE on $\mathrm{PdCu}(110)$. Ts denotes the transition states. The bold arrows indicate the $\mathrm{C}-\mathrm{Cl}$ bond which is being cleaved.

Figure I-9. Two-dimensional potential energy surface for NO dissociation on the Pd surface; (a) and (b): the most stable adsorption sites; (c) and (d): light gray sphere represents the $\mathrm{N}$ atom and dark gray sphere represents the $\mathrm{O}$ atom; (e): site dimensions; (f) reaction path for NO dissociation and diffusion. $\mathrm{T}_{1}$ and $\mathrm{T}_{3}$ : dissociation transition states, $\mathrm{T}_{2}$ and $\mathrm{T}_{4}$ : diffusion transition states. 
Figure I-10. Mulliken overlap populations between atoms (the relative changes of the metal-metal bonds are shown as percentages). 28

Figure II-1. Cluster model of Fe(110) surface. (A) Ball and stick model. (B) Spacefill model................................................................................................... 40

Figure II-2. $F e(110)-p(3 \times 2)$ supercell used in a PBC model of the Fe(110) surface. Rectangle box represents a $\mathrm{p}(1 \times 1)$ unit cell. Numbers represent surface Fe atoms. Atoms in gray represent the second surface layer. Big and small arrows indicate the direction of replication of the supercell and coordinates, respectively.

Figure II-3. Fitting of calculated total energies of bulk iron to the Birch-Murnaghan equation of state. 43

Figure II-4. The unit cell of the body-centered cubic (bcc) iron (A) and Fe(100) (B), $\mathrm{Fe}(110)(\mathrm{C})$, and $\mathrm{Fe}(111)$ surfaces defined by Miller Indices. Solid lines represent each surface. 45

Figure II-5. $p(1 \times 1), p(2 \times 2)$, and $p(3 \times 2)$ supercells of the $\mathrm{Fe}(110)$ surface. The small and big rectangles represent $p(1 \times 1)$ and $p(2 \times 2)$, respectively. The entire structure is $\mathrm{Fe}(110)-\mathrm{p}(3 \times 2)$. The lighter and darker atoms represent the surface and subsurface layers of $\mathrm{Fe}(110)$, respectively.............................. 46

Figure II-6. $\quad \mathrm{Fe}(110)-\mathrm{p}(3 \times 2)$ surface model with vacuum space of $16 \AA$. An intact TCE molecule is located above the surface. (A) Side view of the model. (B) Top side view of the model..................................................................... 47

Figure II-7. Periodic three-layer slabs of Fe(110)-p(3×2) with adsorbed TCE. .......... 48

Figure III-1. $F e(110)-p(3 \times 2)$ supercell and adsorption sites used for chloroethene adsorption calculations. The rectangle demarcates $p(1 \times 1)$ and the solid line denotes the orientation of the chloroethene $\mathrm{C}=\mathrm{C}$ bond. The lighter and darker atoms represent the surface and subsurface layers of $\mathrm{Fe}(110)$, respectively. (A) atop 1 site. (B) atop 2 site. (C) long-bridge (LB) site. (D) short-bridge (SB) site 54

Figure III-2. Maximum force and relative total energy calculated for TCE adsorption at the short-bridge (SB) site of $\mathrm{Fe}(110)$ during geometry optimization. ...... 56

Figure III-3. Relative adsorption energies of PCE, TCE, and cis-DCE from the gas phase onto the atop 1 site of $\mathrm{Fe}(110)$, for different surface relaxation conditions and different supercell sizes. (A tight convergence criterion $\left(2.57 \times 10^{-1} \mathrm{eV} / \AA\left(10^{-2} \mathrm{Ryd} /\right.\right.$ a.u. $\left.)\right)$ on the forces acting on each relaxed atom of the system and a $5 \times 5 \times 1 k$-point grid were used in the adsorption energy calculations. For geometry optimizations at the atop 1 site, the chloroethene $\mathrm{C}=\mathrm{C}$ bond axis maintains an orientation parallel to the $\mathrm{Fe}$ 
surface, even when the tight convergence criterion on the forces is applied).

Figure III-4. Relative adsorption energies of PCE, TCE, and cis-DCE from the gas phase on the atop 1 site of the $\mathrm{Fe}(110)-\mathrm{p}(3 \times 2)$ supercell for different $k$ grids.

Figure III-5. Adsorbed configurations of PCE, TCE, and cis-DCE on the relaxed $\mathrm{Fe}(110)-\mathrm{p}(3 \times 2)$ surface. (A) PCE at atop 1. (B) TCE at atop 1. (C) cisDCE at atop 1. (D) PCE at long-bridge (LB). (E) TCE at atop 2. (F) cisDCE at short-bridge (SB). 64

Figure III-6. PDOS on $d$ orbitals of the relaxed Fe(110)-p(3×2) surface (A to E), and $p_{z}$ orbitals of carbon and chlorine atoms of PCE (F to I). (A) Clean iron surface with no adsorbates. (B) Fe surface with PCE molecule $5 \AA$ above the surface (i.e., "non-interactive" PCE). (C) Fe surface with adsorbed PCE at atop 1 site. (D) Fe surface with adsorbed TCE at atop 1 stie. (E) Fe surface with adsorbed cis-DCE at atop 1 site. (F) Carbon and (H) chlorine of non-interactive PCE. (G) Carbon and (I) chlorine of adsorbed PCE at the atop 1 site. The solid and dotted lines indicate PDOS for spin-up and spin-down electrons, respectively. The Fermi energy is denoted by the vertical line at $0 \mathrm{eV}$. 69

Figure III-7. The $\alpha$-spin highest occupied molecular orbitals (HOMOs: A to C) and lowest unoccupied molecular orbitals (LUMOs: D to F) of adsorbed PCE, TCE, and cis-DCE at the atop 1 site of the $\mathrm{Fe}_{7}$ cluster model. The two colors represent the positive and negative portions of the wavefunction.

Figure III-8. The $\alpha$-spin second-highest occupied molecular orbitals (HOMO-1) of adsorbed PCE and HOMOs of adsorbed TCE and cis-DCE at the atop 1 site of the $\mathrm{Fe}_{7}$ cluster model. The two colors distinguish the positive and negative portions of the wavefunction. 72

Figure IV-1. $\beta$-elimination pathway for dissociation of the first chlorine from TCE, showing the four images used in the reaction path analysis. (A) Adsorbed TCE, (B) $2^{\text {nd }}$ image, (C) $3^{\text {rd }}$ image, and (D) $1^{\text {st }}$ Cl-dissociated TCE. ...... 79

Figure IV-2. Chloroethene dissociation pathways and activation energies for (A) PCE, (B) TCE, and (C) cis-DCE 83

Figure IV-3. Energy diagram for TCE dechlorination (Pathway [6] and [7] in Figure IV-2). TS and Int denote transition states and intermediates, respectively. The energy differences between the adsorbed reactant, intermediates, and product are reported in $\mathrm{kJ} / \mathrm{mol}$. The viewing angle and orientation used for 
the coordinate system in the images is shown in the top right-hand corner.

Figure IV-4. Energy diagram for the PCE dechlorination (Pathway [1] and [2] in Figure IV-2). The nomenclature is the same as is used in Figure IV-3. 89

Figure IV-5. Energy diagram for the cis-DCE dechlorination (Pathway [11] and [12] in Figure IV-2). The nomenclature is the same as is used in Figure IV-3... 90

Figure IV-6. Comparison between theoretical and experimental vibrational frequencies (expressed as wavenumbers, $\mathrm{cm}^{-1}$ ) of gas-phase TCE. EXP represents the experimental data. G03 and QE are the theoretical calculations obtained from the cluster model (Gaussian 03) and the periodic model (Quantum Espresso). Theoretical values calculated by Zhang et al. are also shown.

Figure IV-7. Rate constants for the most favored pathways of PCE, TCE, and cis-DCE dechlorination (A) and the pre-exponential factors ( $A$ factors) of the ratelimiting steps (B). The numbers in square brackets denote the reaction pathways labelled in Figure IV-2 ......................................................... 94

Figure IV-8. Projected density of states (PDOS) for the clean Fe surface with noninteractive PCE (A) and the transition states of PCE (B), TCE (C), and cisDCE (D). The black solid line and dotted line represent the spin-up and spin-down Fe $3 d$ states, respectively. The red, blue, and green solid lines represent $\mathrm{Cl} 2 p_{z}$ states according to the number marked on the transition state configurations of each adsorbate. Non-bonded $\mathrm{Cls}$ that are farthest from the $\mathrm{C} \equiv \mathrm{C}$ bond are the first dissociated and adsorbed $\mathrm{Cls}$ on the $\mathrm{Fe}$ surface. The other non-bonded $\mathrm{Cls}$ are the transition states of the chloroethenes. The Fermi energy is denoted by the vertical line at $0 \mathrm{eV}$.

Figure IV-9. Charge densities for the transition states corresponding to the second chlorine dissociation from adsorbed PCE (A \& B), TCE (C \& D), and cisDCE (E \& F) on the Fe(110) surface. The first row represents the adsorbed chloroethenes viewed from above. The second row shows the side views perpendicular to the solid lines shown in the first row (arrows indicate the direction of the viewpoint). The numbers shown in the chlorine atoms are the same as those in Figure IV-8 99 


\section{List of Tables}

Table I-1. The most stable adsorption sites of adsorbates on $\mathrm{Fe}(110)$ and $\mathrm{Fe}(100)$ surfaces. .............................................................................................. 15

Table III-1. Adsorption energies of chloroethenes from the gas phase and the aqueous phase $^{a}$ on $\mathrm{Fe}(110)-\mathrm{p}(3 \times 2)$ surface with a $2 \times 3 \times 1 \quad k$-point grid ................. 63

Table III-2. Bond lengths $(\AA)$ and dihedral angles $\left(^{\circ}\right)$ of chloroethenes in the gas phase and adsorbed on the atop 1 site of the relaxed $\mathrm{Fe}(110)-\mathrm{p}(3 \times 2)$ surface.... 65

Table IV-1. Comparison of theoretical and experimental vibrational frequencies (expressed as wavenumbers, $\mathrm{cm}^{-1}$ ) for gas-phase TCE. The column titles

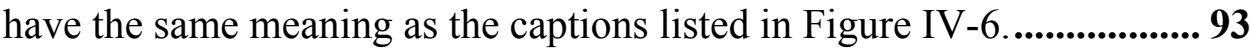




\begin{abstract}
This study investigated the adsorption and dissociation of perchloroethene (PCE), trichloroethene (TCE), and cis-dichloroethene (cis-DCE) on zerovalent iron to evaluate the relative reactivity of these compounds on zerovalent iron. The mechanisms by which iron decomposes chlorinated solvents by catalytic cleavage of the carbon-chlorine bond, are as not yet well understood. To develop process models for the optimal design of in situ and ex situ zerovalent iron treatment systems for the removal of chlorinated solvents from drinking water supplies, it is important to understand these mechanisms, and in particular how the degree of chlorination of the contaminant affects its reactivity on the iron surface. The Fe(110) surface was selected for this study because it is one of mostly commonly observed surfaces and is the most densely packed and thermodynamically stable iron surface. Periodic density functional theory (DFT) and the generalized gradient approximation (GGA) were used to determine the most thermodynamically favorable site on $\mathrm{Fe}(110)$ for the adsorption of all three chloroethenes. The climbing image nudged elastic band (CI-NEB) method with the periodic DFT and the GGA was employed to calculate activation energies of the chloroethene compounds according to the principal dechlorination mechanism of reductive $\beta$-elimination. The dechlorination rate constants of the chloroethenes were estimated using an Arrhenius equation with theoretically calculated vibrational frequencies of the compounds. Of the adsorption sites examined, an atop site, where the chloroethene $\mathrm{C}=\mathrm{C}$ bond straddles a surface iron atom, was the most energetically favorable site for the adsorption of all three chloroethenes. Electronic structure and property analyses demonstrate the strong hybridization of the $\pi$-bonding orbital between the chloroethene $\mathrm{C}=\mathrm{C}$ bond and the iron surface suggesting that adsorbed chloroethenes are strongly activated on $\mathrm{Fe}(110)$ and are likely precursors for subsequent chloroethene dissociation on the Fe surface. Taking into account the effect of solvation indirectly, the ordering of the adsorption energies of chloroethenes from the aqueous phase onto $\mathrm{Fe}(110)$ is in agreement with experimental observation $(\mathrm{PCE}>\mathrm{TCE}>$ cis-
\end{abstract}


DCE). Chloroethenes with a higher number of chlorine atoms have lower activation energies than those with fewer chlorine atoms. The activation energies of PCE, TCE, and cis-DCE at their reaction rate-limiting steps are $9.9,16.6$, and $23.8 \mathrm{~kJ} / \mathrm{mol}$, respectively. At room temperature $(300 \mathrm{~K})$, for example, the dechlorination rate of PCE is 14 times and 338 times faster than those of TCE and cis-DCE, respectively. Details of the electronic properties of the adsorbed configurations and the transition states of the three chloroethenes are also presented. 


\section{CHAPTER I \\ INTRODUCTION AND BACKGROUND}

\section{Introduction}

\subsection{Motivation and problem overview}

The anthropogenic production, release, and disposal of chlorinated solvents have caused severe contamination of the environment. The common chlorinated solvents are highly chlorinated compounds such as perchloroethene (PCE, $\left.\mathrm{C}_{2} \mathrm{Cl}_{4}\right)$ and trichloroethene (TCE, $\mathrm{C}_{2} \mathrm{HCl}_{3}$ ). PCE and TCE are the most commonly-found contaminants at hazardous waste disposal sites due to widespread use and subsequent disposal $(1,2)$. These substances cause irritation of the skin, eyes, kidneys, and respiratory tract; in particular TCE is reasonably anticipated to be a human carcinogen (3). Chloroethenes that are released into the subsurface penetrate into the water table and persist as a long-term source of contamination in the subsurface environment $(4,5)$.

Recently, zerovalent iron $\left(\mathrm{Fe}^{0}\right)$ in particular has gained widespread acceptance as an effective material for groundwater remediation at sites contaminated with chloroethenes (6-8), especially because of its low cost and nontoxic properties (9). However, the exact mechanisms by which iron decomposes chlorinated solvents (i.e., dechlorinationcatalytic cleavages of the carbon-chlorine bond (9)) are not well understood. In order to design treatment systems to remove chlorinated solvents from drinking water supplies, it is important to understand these mechanisms, and in particular how the degree of chlorination of the pollutant affects its reactivity on the iron surface. Understanding the dechlorination rates and pathways is essential to the development of process models that optimize the design of in-situ and ex-situ treatment systems for the removal of chloroethenes from drinking water supplies (10).

It is generally assumed that reactivity of chloroethenes should increase with increasing number of chlorines ( 6 ), as was recently reported for chloroethene decomposition on iron $(11,12)$ and zinc $(10)$. The dechlorination rate of PCE is 5.4 times 
faster on zerovalent iron $(11,12)$, and 170.2 times faster on zerovalent zinc $(10)$ than that of TCE. The higher degree of chlorination also favors rapid dechlorination on ironbearing soil minerals (biotite (13), magnetite (14), and green rust (15)). However, the reverse trend has also been reported for decomposition of chloroethenes by iron (6), palladium (16), and iron sulfide (17). The overall order of reactivity for reduction by zerovalent iron was reported to be: vinyl chloride $(\mathrm{VC})>c i s$-dichloroethene $($ cis-DCE) $>$ TCE > PCE (6). The same trend was shown in the dechlorination of chloroethenes by palladium metal catalysts ( $\mathrm{Pd} / \mathrm{Al})$ with hydrogen gas (16). TCE was also transformed 2.6 times faster than PCE by iron sulfide (17). Hence the reactivity trend for these compounds remains controversial.

Reasonable explanations have been suggested for these contradicting results with respect to the effect of chlorination on the relative reactivity of chloroethenes with $\mathrm{Fe}^{0}$. One of the most plausible assumptions is that carbon impurities included in iron may serve as nonreactive sites on the iron surface where chloroethenes may be adsorbed without further reaction. The adsorbed amount of chloroethenes is misinterpreted as a part of the overall reaction rate (18). Highly chlorinated species, e.g., PCE, show greater adsorption than other less-chlorinated species, indicating that the adsorption of PCE may easily be mistaken for rapid reaction $(6,18)$. Despite this putative explanation, it remains unresolved how the degree of chlorination affects the relative reactivity of chloroethenes on the iron surface, due to the difficulty of performing quantitatively accurate experiments.

In the presence of zerovalent iron, reductive dechlorination of chloroethenes occurs through $\beta$-elimination, hydrogenolysis, $\alpha$-elimination, and hydrogenation $(6,14,15,19)$. Reductive elimination removes two halide ions whereas hydrogenolysis replaces a halogen with a hydrogen atom. Both reductive elimination and hydrogenolysis are accompanied by a net transfer of two electrons (20). Reduction of PCE, TCE, cis-DCE and trans-DCE to acetylene by reductive $\beta$-elimination is strongly kinetically favored over reduction to $\mathrm{VC}$ and ethene by hydrogenolysis $(6,14,15,21)$. Arnold and Roberts (6) reported that the reductive $\beta$-elimination accounts for $87 \%$ of PCE, $97 \%$ of TCE, $94 \%$ of cis-DCE, and $99 \%$ of trans-DCE reaction that produce dichloroacetylene, chloroacetylene, and acetylenes, respectively. 
In this study, density functional theory (DFT) methods are applied to investigate the relative reactivity of chloroethenes on the iron surface. This study performs tasks including: determining the most stable adsorption configurations of chloroethenes and analyzing the dechlorination pathways of chloroethenes on the iron surface. In detail, the determination of the adsorption configurations of chloroethenes is the first necessary step for analyzing the dechlorination pathways because chemical reactions between chloroethenes and the iron surface are initiated after the molecules are stably placed on the most favorable sites on the iron surface. The activation energies of chloroethenes (i.e., the required energy for chloroethenes to be dechlorinated) are then computed in the dechlorination pathways. This analysis of the activation energies of chloroethenes will help not only close knowledge gaps in our understanding of dechlorination pathways, but also confirm or discard hypotheses concerning the relative reactivity of chloroethenes on the zerovalent iron. 


\subsection{Purpose of study}

The relative reactivity of chloroethenes on zerovalent iron is investigated using density functional theory (DFT) methods to improve the design of in situ and ex situ treatment systems for the removal of chloroethenes from drinking water supplies. Differentiation between adsorption and dissociation steps is necessary to prevent misinterpretation of chloroethene adsorption as a part of the overall dechlorination rate. The relative order of reactivity among the chlorothenes is determined by achieving two goals as follows.

Determination of adsorption configurations of chloroethenes. The adsorption configurations of chloroethenes on the $\mathrm{Fe}(110)$ surface are investigated to determine the thermodynamically favorable adsorption sites of PCE, TCE, and cis-DCE on the iron surface. This should be done as a first step of accurate characterization of the adsorption sites and energies toward elucidating the effect of chlorination on the relative reactivity of chloroethenes on zerovalent iron. The relative order of adsorption energies of chloroethenes on the $\mathrm{Fe}(110)$ surface is determined from the aqueous phase by accounting for hydration energies of chloroethenes in bulk water.

Determination of activation energies of chloroethenes. Using the adsorbed configurations of the chloroethenes, the activation energies of chloroethenes on the $\mathrm{Fe}(110)$ surface are determined based on assumed dechlorination pathways governed by reductive $\beta$-elimination. The activation energies are converted into the dechlorination reaction rate constants using vibrational frequencies of reactants and transition states of chlorothenes in the dissociation pathways. This helps not only close the knowledge gap in our understanding of dechlorination pathways, but also confirm or discard hypotheses concerning the relative reactivity of chloroethenes on the zerovalent iron.

This study therefore presents and discusses theoretical results from DFT calculations for the adsorption energies and the activation energies in the dechlorination pathways of chloroethenes. Electronic properties of chloroethenes adsorbed and dissociated on the Fe(110) surface are also presented. 


\section{Literature Review}

\subsection{Degradation pathways for chloroethenes}

\subsubsection{Dechlorination mechanisms}

Multiple mechanisms exist for the dechlorination of chloroethenes. Three wellknown routes for the transformation of chloroethenes are 1) anaerobic reductive dechlorination by halorespirers; 2) aerobic degradation by epoxidation; and 3) abiotic reductive dechlorination by transition metals. For in-situ remediation, the mechanisms interact in a complex fashion to achieve degradation of chloroethenes. Although the interplay between these competing mechanisms has not been fully explored, the individual reaction pathways have been widely studied. Under anaerobic conditions, halorespirers are able to dechlorinate PCE, TCE, DCEs and VC. The endproduct of the reductive dechlorination is ethene which subsequently reacts to ethane. Dehalococcoides, for example, is used for in-situ dechlorination in bioaugmentation schemes where its growth is stimulated by the supply of an electron donor such as acetate and molecular hydrogen (22). In addition, Dehalococcoides ethenogenes strain 195 is capable of respiratory reductive dechlorination of PCE completely to ethane with $\mathrm{H}_{2}$ as an electron donor (23). Dehalobacter restrictus and Dehalospirillum multivorans can catalyze the reductive dechlorination of $\mathrm{PCE}$ and TCE to cis-DCE with $\mathrm{H}_{2}$ and reduced methyl viologen as an electron donor $(24,25)$. Figure I-1 shows the reaction pathway for anaerobic reductive dechlorination by halorespirers.

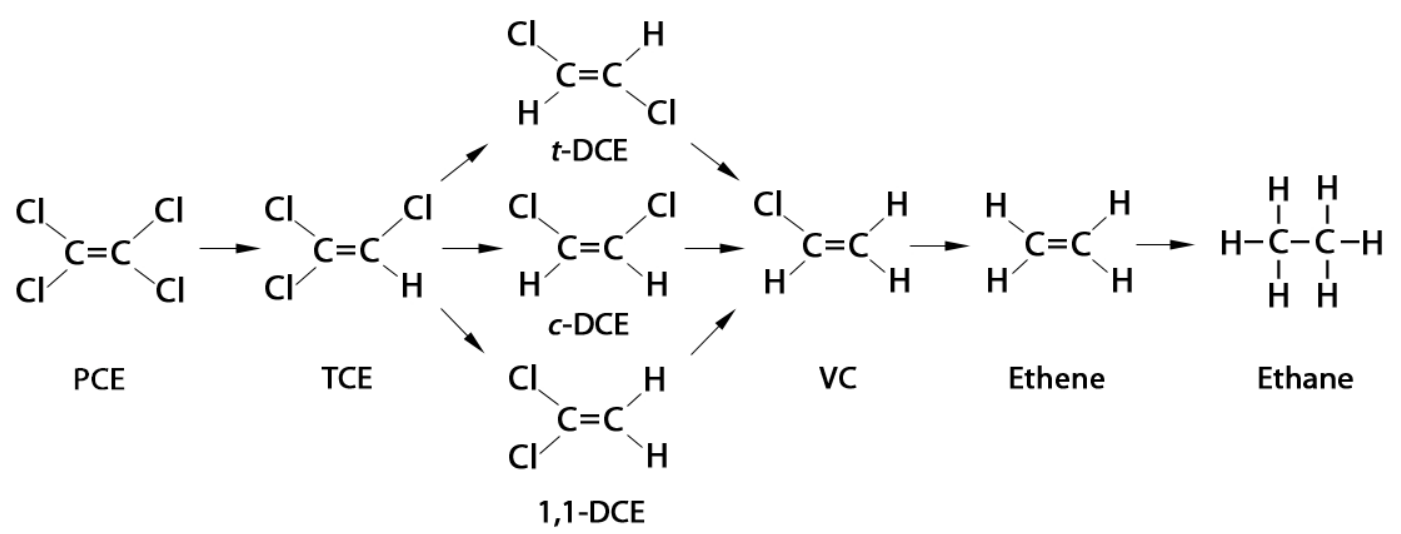

Figure I-1. Reaction pathways for anaerobic reductive dechlorination of PCE 
Aerobic degradation putatively occurs by epoxidation (26), through which a threemembered ether with two carbon and one oxygen atom forms, leading to mineralization of chloroethenes to $\mathrm{CO}_{2}$ (Figure I-2). Epoxidation usually occurs cometabolically by nonspecific binding of various oxygenases, though recent studies have shown that VC $(27,28)$ and cis-DCE (29) can be utilized as sole growth substrates.

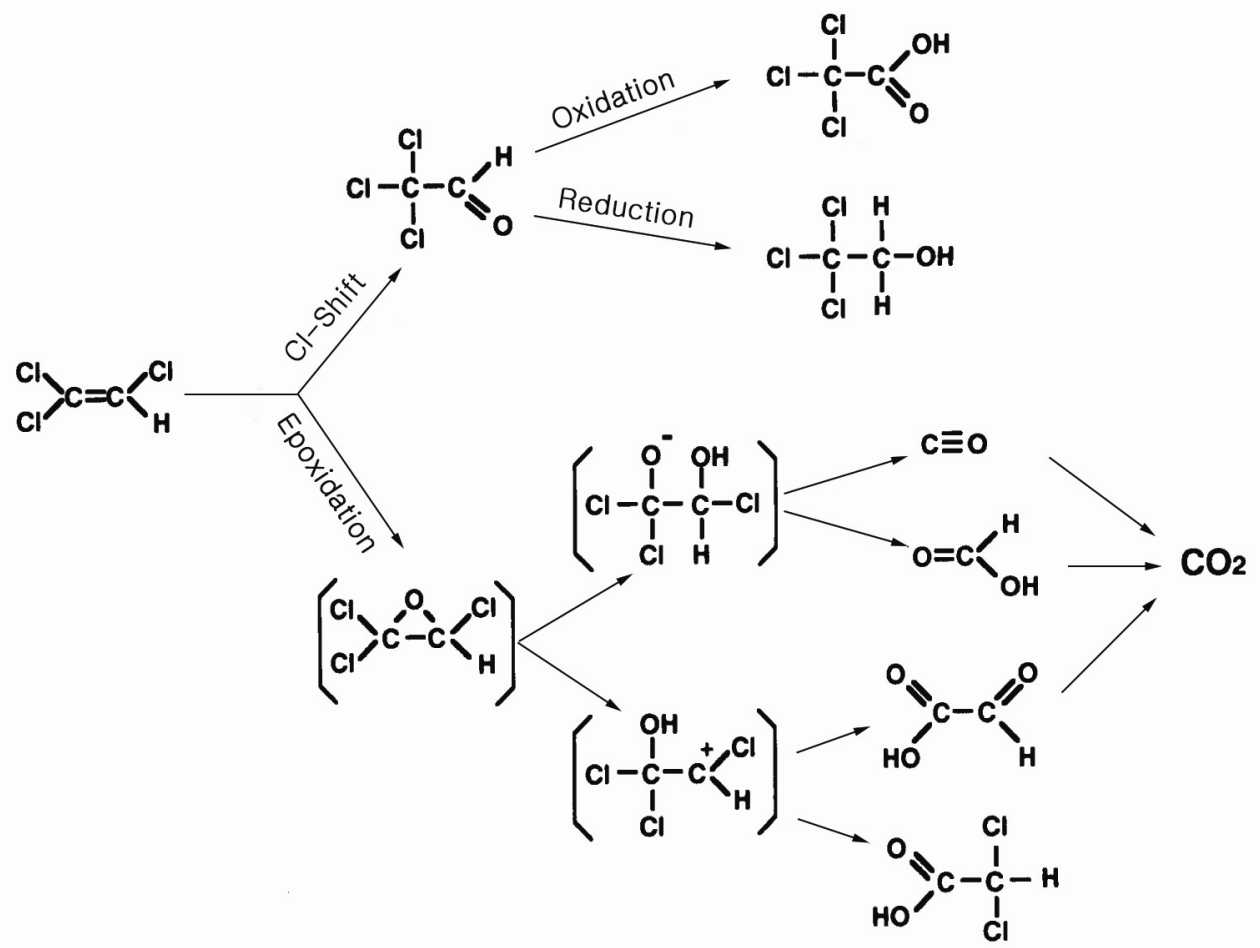

Figure I-2. Reaction pathway for aerobic oxidation of TCE (adapted from Nakajima et al. (30)).

A third mechanism of degradation of chloroethenes is abiotic reductive dechlorination by transition metals. In the presence of zerovalent iron, reductive dechlorination of chloroethenes occurs through $\beta$-elimination, hydrogenolysis, $\alpha$ elimination, and hydrogenation $(6,14,19)$. Reductive elimination removes two halide ions whereas hydrogenolysis replaces a halogen with a hydrogen atom. Both reductive elimination and hydrogenolysis are accompanied by a net transfer of two electrons (20). Reduction of PCE, TCE, cis-DCE and trans-DCE to acetylene by reductive $\beta$-elimination is strongly kinetically favored over reduction to $\mathrm{VC}$ and ethene by hydrogenolysis $(6,14$, 
21). Iron-bearing soil minerals such as iron sulfides and polysulfides (pyrite, marcasite, troilite, and mackinawite) also can contribute to abiotic reduction of chloroethenes and chloroethanes $(13,14,17,31)$. Surface Fe(II) or sulfide species are assumed responsible for these reactions $(13,31)$. Figure I-3 illustrates the hypothesized reaction pathways for the abiotic reductive dechlorination on zerovalent iron surface.

Matheson and Tratnyek (9) surmised that the three major reductants in a zerovalent iron-water system are elemental iron, ferrous iron and hydrogen that result from iron corrosion. The first pathway involves a zerovalent iron surface that undergoes oxidation by water, causing a direct electron transfer from the surface to the chlorinated compound (Equation 1 and Figure I-4 (a)):

$$
\mathrm{Fe}^{0}+\mathrm{RCl}+\mathrm{H}^{+} \rightarrow \mathrm{Fe}^{2+}+\mathrm{RH}+\mathrm{Cl}^{-}
$$

The second pathway involves ferrous iron $\left(\mathrm{Fe}^{2+}\right)$ formed by iron corrosion with water. Dissolved $\mathrm{Fe}^{2+}$ serves as a reductant, although the dechlorination rate is quite slow (32) (Equation 2 and Figure I-4 (b)):

$$
2 \mathrm{Fe}^{2+}+\mathrm{RCl}+\mathrm{H}^{+} \rightarrow 2 \mathrm{Fe}^{3+}+\mathrm{RH}+\mathrm{Cl}^{-}
$$

The third abiotic pathway involves hydrogen produced as a product of iron corrosion with water (Equation 3 and Figure I-4 (c)). For this pathway, a transition metal surface or surface defect is required to catalyze the reaction (9):

$$
\mathrm{H}_{2}+\mathrm{RCl} \rightarrow \mathrm{RH}+\mathrm{H}^{+}+\mathrm{Cl}^{-}
$$




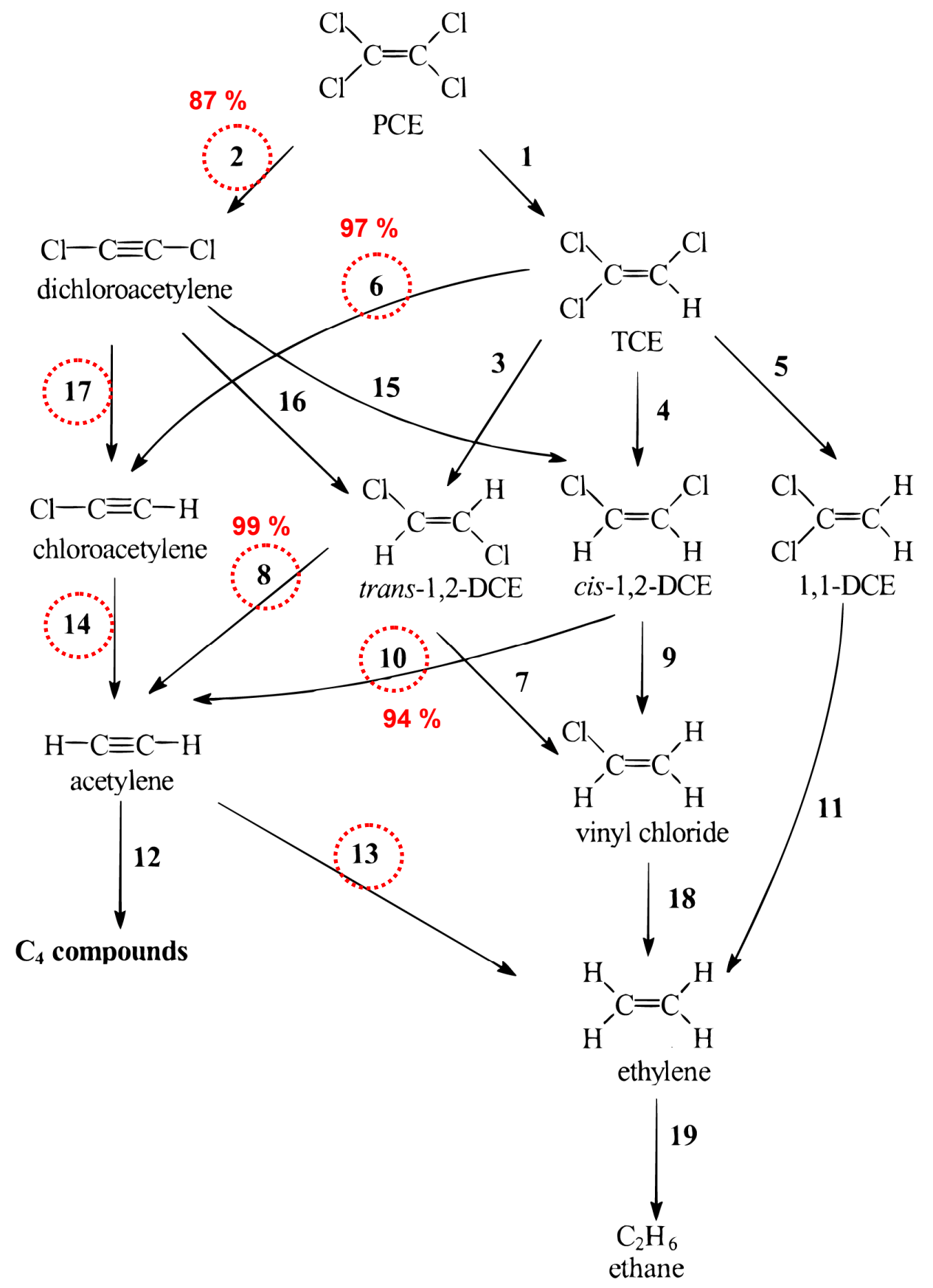

Figure I-3. Hypothesized reaction pathways for abiotic reduction of PCE on zerovalent iron. Numbers correspond to each reaction. Reductive $\beta$-elimination: $2,6,8$ and 10; hydrogenolysis: $1,3,4,5,7,9,14,17$ and 18; $\alpha$-elimination: 11 ; hydrogenation: 13,15 , 16 and 19. Dotted circles indicate the kinetically-favored pathway for reduction of each product. Percentages represent the amount of product transformed through the pathway (adapted from Arnold and Roberts (6)). 
A)

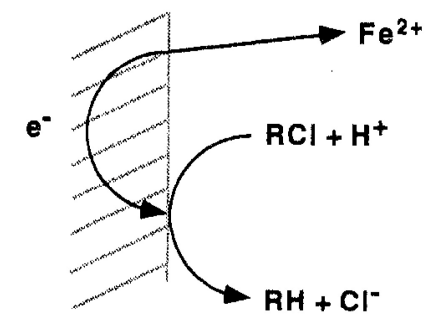

B)

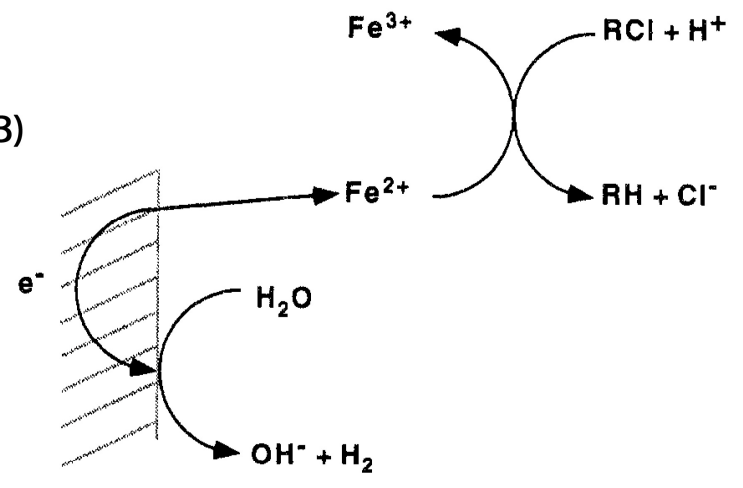

C)

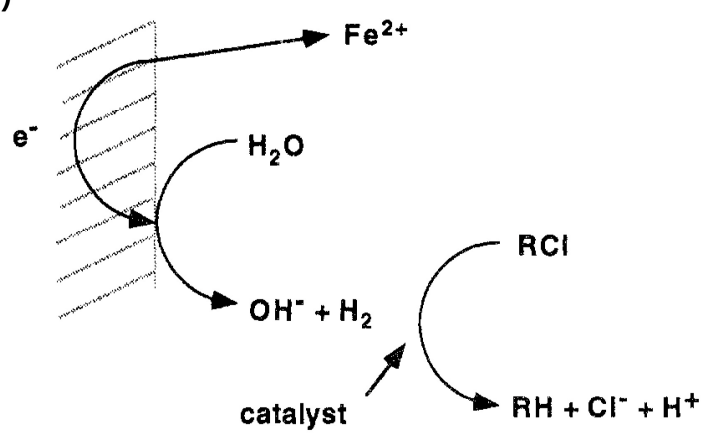

Figure I-4. Reaction pathways for reductive dechlorination in anoxic aqueous system (adapted from Matheson and Tratnyek (9)).

Arnold and Roberts (6) hypothesized mechanisms for PCE degradation with iron particles. As can be seen in Figure I-5, PCE is adsorbed on the iron surface with $\pi$ bonding caused by nonspecific (e.g., hydrophobic) interaction. In the next step, the $\pi$ bonded intermediate forms a di- $\sigma$-bonded surface-adsorbed species. With this adsorbed form of PCE, two possible reactions may occur: reductive $\beta$-elimination and hydrogenolysis. Through reductive $\beta$-elimination, two halide ions are dissociated from the di- $\sigma$-bonded intermediate during two successive steps resulting in the formation of acetylene. In the other pathway, a hydrogen atom is attached to the di- $\sigma$-bonded intermediate from the source of adsorbed hydrogen on the iron surface and a halide ion is eliminated. 
1)<smiles>ClC(Cl)=C(Cl)Cl</smiles><smiles>[Y]C1C=CC=C1[Te]</smiles><smiles>ClC1=CCC(Cl)=C1Cl</smiles>

2)<smiles>ClC(Cl)=C(Cl)Cl</smiles>

3a)
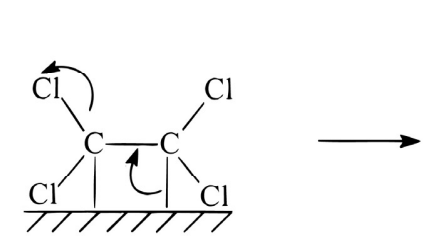

4a)<smiles>ClC1(Cl)CC1(Cl)Cl</smiles>

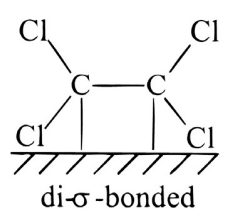

$\pi$-bonded

\section{3a)}

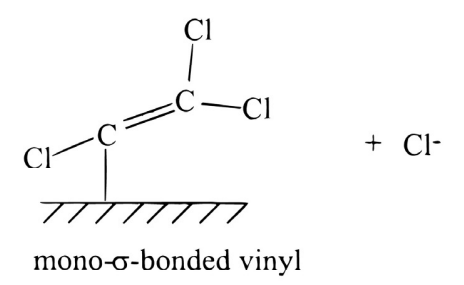

3b)
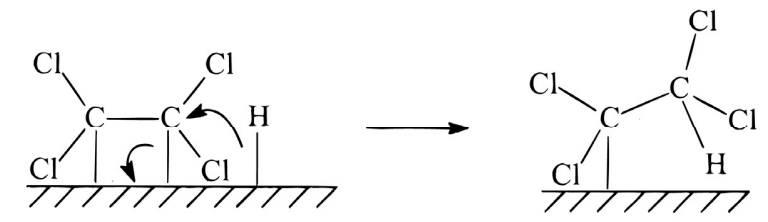

mono- $\sigma$-bonded alkyl

4b)<smiles>ClC(Cl)C[C@@H](Cl)C(Cl)Cl</smiles>

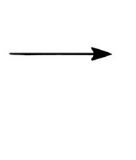<smiles>ClC=C(Cl)Cl</smiles>

Figure I-5. Hypothesized mechanisms for dechlorination of PCE. Reductive $\beta$ elimination may proceed via steps $1,2,3 \mathrm{a}$ and $4 \mathrm{a}$ while hydrogenolysis proceeds via steps 1, 2, 3b and 4b (adapted from Arnold and Roberts (6)). 


\subsubsection{Applications of abiotic dechlorination}

Zerovalent iron has been widely used for in situ and ex situ remediation strategies at sites contaminated with organohalides or oxidized metal ions $(6,7)$. Permeable reactive barriers (PRBs) as well as the traditional pump and treat method have been extensively adapted using iron for the remediation, especially because of its economic value and nontoxic properties $(6,9)$ and due in part to the difficulties often encountered in biotic treatment. Remediation schemes for PRBs involve installing reactive material in the subsurface of a contaminated site through which contaminated groundwater flows, and degrading the contaminants in situ as opposed to pump and treat methods where contaminated groundwater is pumped to the surface, passed through a treatment system and reinjected belowground. In addition to haloalkanes and haloalkenes, zerovalent iron has been used to dehalogenate more complex halogenated compounds such as polychlorinated biphenyls (PCBs), 1,1,1-trichloro-2,2-bis( $p$-chlorophenyl)ethane (DDT), and polybrominated diphenyl ethers (PBDEs) (33-35).

The use of zerovalent iron in PRBs for in situ groundwater remediation has rapidly gained acceptance as a cost-effective technology. Once installed, although case-by-case field studies may show different results, PRBs may function for 5 to 10 years without replacement of the reactive materials (36). Minimum maintenance is required for operation of PRBs compared to biotic treatment methods. However, the successful degradation of halogenated organics using PRBs strongly depends on environmental conditions in the subsurface, because dechlorination is most effective under anaerobic conditions that prevent corrosion of the iron by oxygen (37). If iron is exposed to oxygen, iron metal is oxidized, releasing electrons that can be used in the reduction of water to generate hydroxide ions (38). These hydroxide ions can form insoluble metal hydroxides that coat the exterior surface of the iron and reduce its reactivity (38). Furthermore, the hydroxide ions produced by iron corrosion increase water $\mathrm{pH}$ and react with dissolved carbonic acid $\left(\mathrm{H}_{2} \mathrm{CO}_{3}\right)$ and bicarbonate $\left(\mathrm{HCO}_{3}{ }^{-}\right)$, forming carbonate ions $\left(\mathrm{CO}_{3}{ }^{2-}\right)$ that precipitate solid carbonates onto the iron surface, further reducing the reaction rate $(36$, 37). 


\subsection{Adsorption of chloroethenes on metal surfaces}

The transformation of halogenated compounds on transition metal surfaces has been an important environmental issue not only because of ground water contamination but also because of ozone layer destruction. The catalytic cleavage of the carbon-halogen bond and its replacement with the carbon-hydrogen bond in the halogenated compounds is fundamental to the destruction of these compounds (39-41). Reductive dechlorination of chlorinated contaminants may occur via three steps, involving adsorption of chlorinated compounds onto the metal surface; surface-catalyzed reduction; and desorption of the dechlorinated products from the metal surface. An accurate characterization of the adsorption site, adsorption modes and packing arrangement are important initial steps in the analysis of dechlorination on metal surfaces (42).

The surface-catalyzed reactions of many adsorbates and metals have been investigated, including important chemical processes such as hydrogen chemisorption on iron for iron-catalyzed ammonia synthesis (43); organic molecules on iron for coatings, adhesive bonding and corrosion inhibition (44); carbon monoxide on transition metal surfaces for methanation and aliphatic hydrocarbon synthesis (45); simple hydrocarbons on transition metals for catalytic combustion (46); and methanol on platinum for fuel cell technology (47). Most experimental and theoretical studies of the use of adsorbates on transition metals have been performed under gas-phase conditions. Theoretical simulations often ignore the effects of water on transition metal surface- catalyzed reactions. Unfortunately, little is known about the adsorption of cis-DCE on zerovalent iron. Thus, an extensive literature review has been conducted consisting of four topics: i) adsorption of chloroethenes on metal surfaces, ii) adsorption of small molecules on iron surface, and iii) the effect of water on the interaction between adsorbates and metal surfaces.

\subsubsection{Di- $\sigma$ - and $\pi$-bond formation}

Two successive steps for the adsorption of PCE on iron were suggested by Arnold and Roberts $(\sigma)$ based on kinetic data. In the initial adsorption step, a $\pi$-bond forms by the sideways overlap of two parallel $p$ orbitals between PCE and the iron surface. In the second step, di- $\sigma$-bonds are formed from the $\pi$-bonded intermediate form. Arnold and 
Roberts noted that the $\pi$-bond formation is supported by the trend toward increasing adsorption with increasing halogenation for the chloroethenes. This trend can be explained by nonspecific (e.g., hydrophobic) interactions and is consistent with the decrease in ionization potential for the chloroethenes with increasing halogenation. Since the inductive effect of the electronegative chlorine (electron withdrawal from $\pi$-system) is outweighed by negative mesomeric effects of chlorine substituents (electron donation), PCE has the lowest ionization potential among the chloroethenes (48).

Di- $\sigma$ - and $\pi$-bond formation between chloroethenes and metal surfaces has been examined in various experimental and theoretical studies. Barbosa et al. (39) investigated the chemisorption of TCE on $\mathrm{PdCu}(110)$ alloy using periodic density functional theory and concluded that both di $-\sigma$ and $\pi$ modes are the most stable configurations. The $\mathrm{C}=\mathrm{C}$ bond of di- $\sigma$ adsorption configuration is more elongated than that of the $\pi$ configuration, which indicates the di- $\sigma$ configuration is more activated. The dihedral angle of $\mathrm{Cl}-\mathrm{C}-\mathrm{C}-\mathrm{Cl}$ changes from $180^{\circ}$ (in gas-phase PCE) to $129^{\circ}$ after adsorption, indicating the extent of $s p^{2}-s p^{3}$ hybridization of the carbon atoms. Barbosa et al. (39) noted that the interaction of TCE on Pd atoms is similar to that of ethene in terms of the perturbation of the $\mathrm{C}=\mathrm{C}$ bond. Jugnet et al. (49) observed at low temperatures $(\leq 180 \mathrm{~K})$ using high resolution electron energy loss spectroscopy (HREELS) that the $\mathrm{C}=\mathrm{C}$ bond of trans-DCE interacts strongly with $\mathrm{Cu}(110)$ surface. The frequencies of the stretching vibrations of the $\mathrm{C}-\mathrm{H}$ and $\mathrm{C}-\mathrm{Cl}$ bonds are lower in the chemisorbed phase than in the gaseous or condensed-phase, indicating that the $s p^{2}$ bonding of the carbon atoms is modified by chemisorption to resemble an alkane-like $s p^{3}$ binding. The authors concluded that the adsorption of trans-DCE on $\mathrm{Cu}(110)$ can be described in terms of a di- $\sigma$-adsorbed species. Yang et al. (41) suggested from near-edge X-ray absorption fine structure (NEXAFS) studies that $\pi$-bonding is involved in the adsorption of TCE, cis-DCE and trans-DCE on $\mathrm{Cu}(100)$ at $95 \mathrm{~K}$. The $\mathrm{C}=\mathrm{C}$ bond length of adsorbed chloroethenes changes little (less than $0.02 \AA$ ) compared to that of gas-phase chloroethenes, indicating there is no rehybridization of the $\mathrm{C}=\mathrm{C}$ bond. Cassuto et al. (50) concluded from a NEXAFS and ultraviolet photoelectron spectroscopy (UPS) study that PCE, TCE, and 1,1-DCE, cisDCE and trans-DCE are weakly bonded (physisorbed) to $\mathrm{Pt}(111)$ and $\mathrm{Pt}(110)$ surfaces at $95 \mathrm{~K}$ without dissociation, while ethene is adsorbed with a di- $\sigma$-bond at this temperature. 
Grassian and Pimentel (51) reported using HREELS that the $\mathrm{C}=\mathrm{C}$ bond of cis- and transDCE opens at low temperature $(110 \mathrm{~K})$ to form a di- $\sigma$-bond on $\operatorname{Pt}(111)$ surface, replacing the alkene $s p^{2}$ bonding with an alkane $s p^{3}$ bonding.

\subsubsection{Adsorption sites of iron surfaces}

As an inexpensive transition metal, iron has been widely used to catalyze reactions involving carbon or hydrogen-containing molecules with sulfur, nitrogen, or other elemental atoms (52). In order to figure out the most stable adsorption sites on iron surfaces using computational methods, some possible adsorption sites are proposed based on insights obtained from experimental results. Then different types of possible adsorption configurations on metal surfaces have been evaluated to find out the most stable configuration of adsorption based on adsorption energy, local geometry of the adsorbed structure, total density of states (DOS) diagram, and electron density analysis. Theoretical studies of iron catalysis have focused on Fe(100), (110) and (111) surfaces. The $\mathrm{Fe}(110)$ structure of body-centered-cubic iron is the most densely packed and thermodynamically stable iron surface (44) and is a useful catalyst, whereas the $\mathrm{Fe}(111)$ surface has a more open structure (53). Figure I-6 shows the high-symmetry adsorption sites for different adsorbate on the surfaces of $\mathrm{Fe}(110)$ and $\mathrm{Fe}(100)$ (52), and Table I-1 summarizes the most preferred adsorption sites for different adsorbates on these two surfaces.

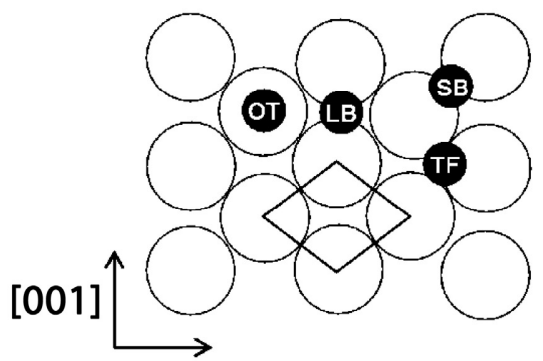

(a) [1]10]

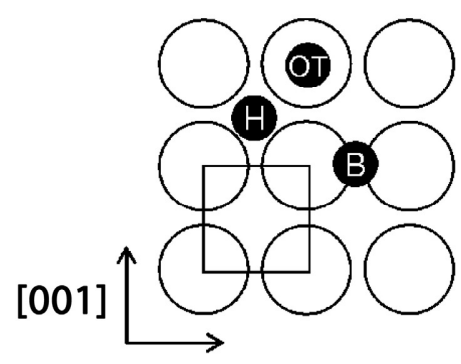

(b) [010]

Figure I-6. High-symmetry adsorption sites on $\mathrm{Fe}(110)$ (a) and $\mathrm{Fe}(100)$ (b) surfaces. OT: on-top position; LB: long-bridge; SB: short-bridge; TF: three-fold; H: hollow; B: bridge. Surface $(1 \times 1)$ cells for both $\mathrm{Fe}(110)$ and $\mathrm{Fe}(100)$ are also shown (adapted from Jiang and Carter (52)). 
Table I-1. The most stable adsorption sites of adsorbates on $\mathrm{Fe}(110)$ and $\mathrm{Fe}(100)$ surfaces.

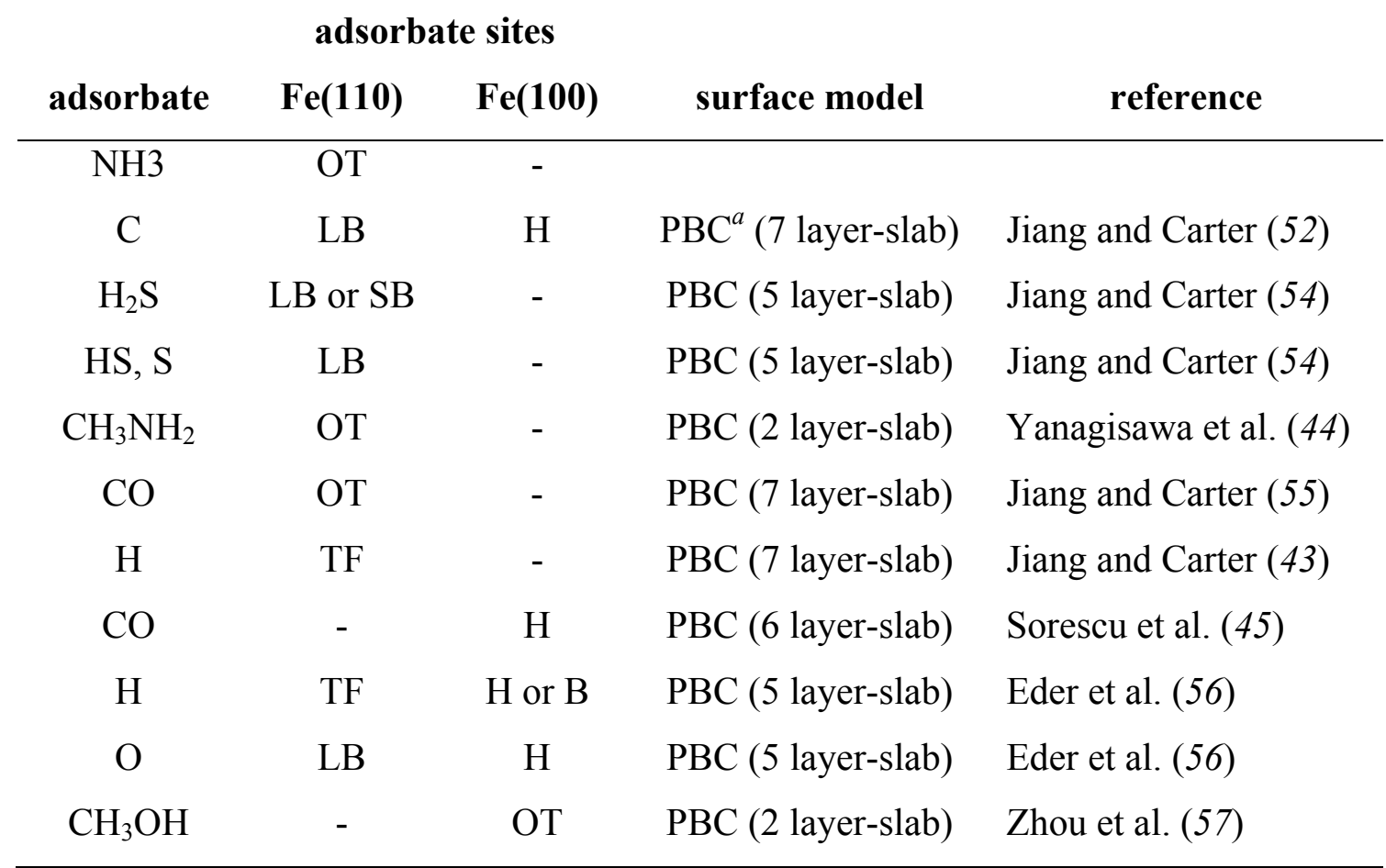

${ }^{a} \mathrm{PBC}$ : periodic boundary conditions

Satoh et al. (53) established that the on-top (OT) site is the most preferred for adsorption of $\mathrm{NH}_{3}$ on both $\mathrm{Fe}(110)$ and $\mathrm{Fe}(111)$. In their calculation, the structure of $\mathrm{NH}_{3}$ was fixed to investigate only the interaction between $\mathrm{NH}_{3}$ and the iron surface. The initial adsorption mode of $\mathrm{NH}_{3}$ was adapted from the experimental results of Benndorf et al. (58) in that $\mathrm{NH}_{3}$ is bonded to the $\mathrm{Fe}(100)$ surface via the nitrogen atom and the $\mathrm{H}$ atoms are pointed away from the surface. For this orientation, the bond distance between the ammonia nitrogen and iron surface was optimized. Satoh and co-authors (53) noted from Mulliken population analysis a considerable transfer of electron population from $\mathrm{NH}_{3}$ to Fe upon on-top adsorption, indicating electron delocalization plays a key role in the adsorption of $\mathrm{NH}_{3}$.

Jiang and Carter (54) found that $\mathrm{H}_{2} \mathrm{~S}$ is weakly adsorbed on the long-bridge (LB) or short-bridge (SB) sites of $\mathrm{Fe}(110)$, while both HS and S are strongly chemisorbed on $\mathrm{Fe}(110)$. Both $\mathrm{H}_{2} \mathrm{~S}$ and $\mathrm{HS}$ are adsorbed on the Fe surface via the sulfur atom, with the hydrogen atom(s) oriented away from the surface. 
Yanagisawa et al. (44) indicated that although the full relaxation of the bcc-Fe surface should be considered in quantitative calculations of the adsorption energies, only the positions of the four central $\mathrm{Fe}$ atoms and the perpendicular positions of the other $\mathrm{Fe}$ atoms in the first layer of the Fe slab need be optimized because the periodic planar frame of the Fe slab should be fixed to preserve the whole surface structure. The authors confirmed that surface relaxation should be taken into account in the calculation of adsorption energies for the Fe surface, because the relaxation lessens the steric repulsion when adsorbates approach to the Fe surface. Yanagisawa and co-authors (44) found that methylamine $\left(\mathrm{CH}_{3} \mathrm{NH}_{2}\right)$ is relatively strongly adsorbed on the OT site of $\mathrm{Fe}(110)$ via the amine nitrogen, which causes electron transfer from the highest occupied molecular orbital (HOMO) of $\mathrm{CH}_{3} \mathrm{NH}_{2}$ to the singly occupied molecular orbital (SOMO) of the $\mathrm{Fe}$ slab. The authors found that the HOMO of the adsorbate corresponds to the lone-pair $p$ orbital of the adsorbing $\mathrm{N}$ or $\mathrm{O}$ atom, whereas the SOMO of the Fe slab corresponds to the $s$ orbital of the Fe atoms. Consequently, the authors concluded that the strength of adsorption of organic molecules depends on their electron-releasing character. By contrast, the methoxy radical $\left(\mathrm{CH}_{3} \mathrm{O}\right)$ is strongly adsorbed on the long-bridge (LB) site of $\mathrm{Fe}(110)$, transferring electrons from the Fe slab to the adsorbate. Zhou et al. (57) reported that $\mathrm{CH}_{3} \mathrm{OH}$ stably adsorbs on the OT site of $\mathrm{Fe}(100)$, also transferring electrons from $\mathrm{Fe}(100)$ surface to the adsorbate molecule. Conversely, in adsorption of $\mathrm{CH}_{3} \mathrm{OH}$ to the $\mathrm{OT}$ site of $\mathrm{Al}(111)$ surface, electron transfer is from the adsorbate to the $\mathrm{Al}$ surface.

\subsubsection{Effect of water molecules}

Taking into account solvent effects is important in metal surface-catalyzed reactions, especially in the investigation of dechlorination of chlorinated compounds by zerovalent iron in groundwater remediation. The thermodynamics and kinetics of surface-catalyzed reactions can be significantly changed under the presence of a solvent medium because partially charged reaction intermediates that are not generally stable in the gas phase can exist in the solution phase and participate in the reaction chemistry (59). Oxidation of methanol on $\mathrm{Pt}(111)$ is a good example of the solvent effect on the oxidation pathways. This reaction has been extensively studied using $a b$ initio simulation because of its essential role in direct methanol fuel cells (DMFC). The oxidation pathway of methanol 
depends strongly on the reaction conditions, particularly on whether it takes place in the vapor phase or in the aqueous phase (47). Neurock et al. (60) studied the pathways of methanol oxidation in both phases in a scheme where in the aqueous phase hydronium ion $\left(\mathrm{H}_{3} \mathrm{O}^{+}\right)$is formed when the adsorbed hydrogen atom on the metal surface, cleaved from the initial methanol oxidation step, desorbs into solution. An extra electron is released when the hydronium forms in the aqueous phase. Consequently, initial methanol oxidation in the vapor phase and in the aqueous phase is expressed as follows (Equation 4 and 5);

$$
\begin{aligned}
& \text { In vapor phase: } \mathrm{CH}_{3} \mathrm{OH}_{(a d s)} \rightarrow \mathrm{CH}_{2} \mathrm{OH}_{(a d s)}+\mathrm{H}_{(a d s)}, \\
& \text { In aqueous phase: } \mathrm{CH}_{3} \mathrm{OH}_{(a d s)}+\mathrm{H}_{2} \mathrm{O}_{(a q)} \rightarrow \mathrm{CH}_{2} \mathrm{OH}_{(a d s)}+\mathrm{H}_{3} \mathrm{O}_{(a q)}^{+}+1 e
\end{aligned}
$$

The authors concluded that water molecules lead the oxidation reaction to the most favored pathway because of the contribution of $\mathrm{H}_{3} \mathrm{O}^{+}$. The activation of the $\mathrm{O}-\mathrm{H}$ bond occurs at $\mathrm{CH}_{2} \mathrm{OH}$ in the vapor phase (endothermic reaction), while the breakage starts at $\mathrm{CHOH}$ in the aqueous phase (exothermic reaction). Figure I-7 illustrates the reaction pathways of both the vapor and the aqueous phases as described by Neurock et al. (60). The bold arrows represent the most favored pathway of methanol oxidation. 


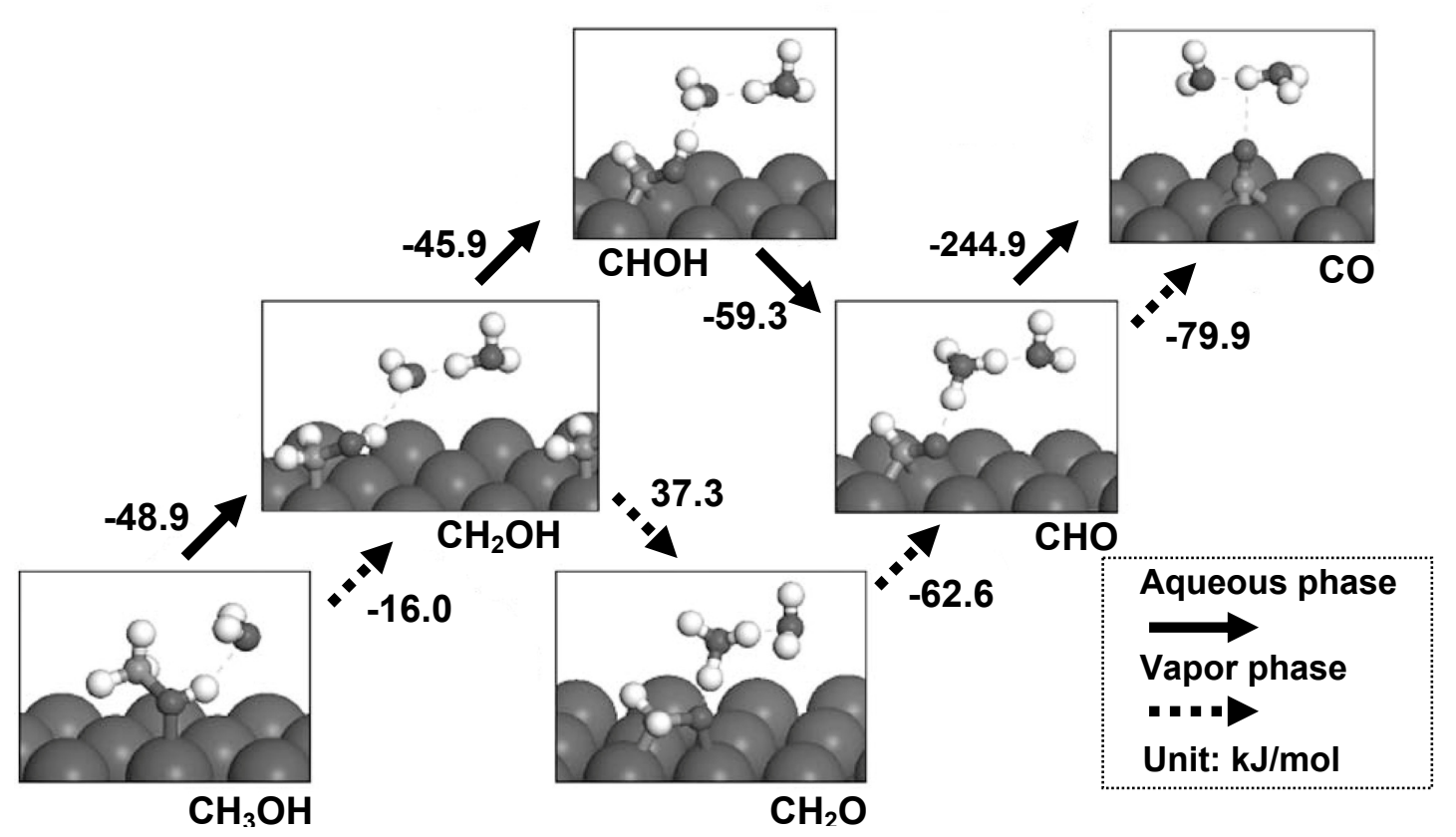

Figure I-7. Potential reaction pathways for methanol oxidation on $\operatorname{Pt}(111)$ in the vapor and the aqueous phases. The solvated products for each step include an $\mathrm{H}_{3} \mathrm{O}^{+}$(aq) group nearby in solution (adapted from Neurock et al. (60)).

Hartnig and Spohr (47) also pointed out the importance of water in methanol oxidation on $\operatorname{Pt}(111)$. In aqueous solution, oxidation is initiated by the formation of a hydrogen bond from the $\mathrm{OH}$ group of the methanol to a solvent molecule. This mechanism is considerably different than any possible gas-phase mechanism of methanol oxidation. Proton transfer to aqueous solution proceeds without an energy barrier and charge delocalization is achieved by the formation of a Zundel ion $\left(\mathrm{H}_{5} \mathrm{O}_{2}{ }^{+}\right)$in aqueous solution.

Okamoto et al. (61) found that the reaction energy for the dehydrogenation of methanol in $\mathrm{Pt}(111) /$ water is $0.7 \mathrm{eV}$ smaller than that of the dehydrogenation of methanol at the $\operatorname{Pt}(111)$ /vacuum interface. The difference in the assumption used in this study, compared to Neurock et al. (60), was that the product hydrogen atoms formed via the dehydrogenation of methanol were assumed to be adsorbed onto the metal surface to make the number of atoms in both the reactant and product the same, thus making it easy to compare the total energy changes.

Solvent effects in other metal surface-catalyzed reactions have been investigated. Desai et al. (59) studied the deprotonation of acetic acid on $\operatorname{Pd}(111)$. The heterolytic 
dissociation of acetic acid is highly endothermic in the gas phase, but nearly thermoneutral in the aqueous phase. Water stabilizes the products, acetate ion and proton, from the dissociation of acetic acid. The authors also found that the interaction of an intermediate with the metal surface is weakened by water molecules. While acetate intermediate is strongly attracted to $\mathrm{Pd}(111)$ surface at $-198 \mathrm{~kJ} / \mathrm{mol}$ without water molecules, the interaction energy decreases to $-114 \mathrm{~kJ} / \mathrm{mol}$ in the presence of water molecules. This water effect is similarly reported from the benzene adsorption study preformed by Schravendijk et al. (62). In the presence of water on Ni(111), a huge barrier prevents benzene from penetrating the water layer and adsorbing onto the $\mathrm{Ni}(111)$ surface.

Teppen et al. (63) studied the dechlorination of TCE in the interlayer space of the clay mineral nontronite in both the absence and the presence of water molecules. It was shown that in the absence of water, TCE is adsorbed flat onto the mineral basal plane without any unusual features compared to unbound TCE; however, in the presence of water, the cis- $\mathrm{Cl}-\mathrm{C}=\mathrm{C}-\mathrm{Cl}$ dihedral angle changes to $52.5^{\circ}$ and the $\mathrm{C}-\mathrm{Cl}$ bond at the $\mathrm{H}-\mathrm{C}-\mathrm{Cl}$ end of TCE significantly elongates indicating the breakage of the $\mathrm{C}-\mathrm{Cl}$ bond. The authors concluded that reductive dechlorination of TCE is more likely to occur in the clay mineral when water is present than when it is not. 


\subsection{Dissociation of chloroethenes on metal surfaces}

Adsorbed chloroethenes on the zerovalent iron surface further react and dissociate. As proposed by Arnold and Roberts (6), two possible dechlorination pathways of cisDCE are reductive $\beta$-elimination and hydrogenolysis (Figure I-3). The former transforms the adsorbed $c i s$-DCE to a mono- $\sigma$-bonded species and then to acetylene, eliminating two chlorine ions; the latter transforms cis-DCE to vinyl chloride by supplying a hydrogen atom and removing a chlorine $(6,10,20)$. The source of the hydrogen atom supplied in hydrogenolysis is most likely from protons reduced by iron (๑). In addition, it is well known that dissociative adsorption of water on clean iron surfaces results in the formation of surface-bound hydroxyl, atomic oxygen, and atomic hydrogen $(9,64)$. The adsorbed hydrogen atoms are possibly directly used for the source of hydrogen in hydrogenolysis and also can combine to form molecular hydrogen (9). These hydrogen molecules can adsorb and dissociate on the iron surface, serving as the source of hydrogen in hydrogenolysis. The scheme of the PCE dechlorination pathways in Figure I-

5 could be used as model for the dechlorination of cis-DCE if the two chlorines bonded to each carbon of PCE are replaced by two hydrogen atoms.

Numerous theoretical studies have been performed to investigate the dissociation of molecules on metal surfaces. These simulations have also recently been used as an invaluable tool to understand experimental results and identify the intermediates of different surface reactions (65). For example, the chemisorption and reaction of nitrogen oxides on metal surfaces has been extensively studied because of the importance of car exhaust catalysts (66). The oxidation of carbon monoxide on metal surfaces has also been widely studied because of air pollution problems. Although experimental and theoretical studies of chloroethenes on iron surfaces have been performed, little specific information is available for the adsorption and dissociation of chloroethenes on iron surfaces. A review of the literature of theoretical studies of molecular dissociation suggests that for relatively large molecules, only the interaction between the adsorbate molecule and the metal surface is typically considered, whereas for smaller molecules, the effect of other adsorbates through the degree of surface coverage is more commonly taken into account. 


\subsubsection{Examples of the investigation of dissociation mechanism}

The investigation of dissociation mechanisms starts with identifying the most favorable surface sites for adsorption of gas-phase species, followed by geometry optimization of stationary points along the dissociation reaction pathways. Once the stationary geometries are found, the transition states between reactants and products are then located. Barbosa and Sautet (67) studied the dechloriantion of TCE on PdCu(110) alloy using a periodic density functional theory. Based on the most favored adsorption configurations of TCE (di- $\sigma$-bonded structures) on $\operatorname{PdCu}(110)$, they found that all intermediate steps are exothermic and all activation energies are relatively small (less than $40 \mathrm{~kJ} / \mathrm{mol}$ ), indicating that the dissociation of TCE on $\mathrm{PdCu}(110)$ is kinetically and thermodynamically favorable. The authors noted that the first cleavage of a $\mathrm{Cl}$ atom is more favored in the $\mathrm{CCl}_{2}$ group rather than in the $\mathrm{CHCl}$ group, which is consistent with the experimental results of $\mathrm{He}$ et al. (68) and Yang et al. (69). The activation energy for the $\mathrm{C}-\mathrm{Cl}$ bond cleavage of the $\mathrm{CHCl}$ group is relatively small $(25 \mathrm{~kJ} / \mathrm{mol})$, which corresponds to the findings of Yang et al. (70) that dissociation of vinyl halides $(\mathrm{C}-\mathrm{Br})$ occurs at low temperatures between $160 \mathrm{~K}$ and $250 \mathrm{~K}$. Interestingly, the $\mathrm{C}-\mathrm{C}$ bond length changes little during the dissociation reaction (approximately $10 \%$ ) relative to the gasphase $\mathrm{C}=\mathrm{C}$ bond length, indicating that both carbon atoms still have $s p^{2}$ hybridization after dissociating. Figure I-8 illustrates the putative dechlorination reaction pathway of di- $\sigma$-bonded TCE on the $\mathrm{PdCu}(110)$ surface. This dissociation model can be applied to that of cis-DCE provided that the $\mathrm{Cl}(3)$ atom in the $\mathrm{CCl}_{2}$ group is replaced by an $\mathrm{H}$ atom (Figure I-8).

The surface reaction of TCE on $\mathrm{PdCu}(110)$ alloy was investigated by Jugnet et al. (65) using HREELS. TCE appears to have a weak interaction with the surface at low temperatures $(180 \mathrm{~K})$. The authors observed that surface reactions occur at $200 \mathrm{~K}$ and most of the $\mathrm{C}-\mathrm{Cl}$ bonds have been broken at $280 \mathrm{~K}$. Between $200 \mathrm{~K}$ and $280 \mathrm{~K}$, both the di- $\sigma$ chemisorbed species and dechlorination intermediates are observed on the surface simultaneously, indicating that dechlorination of TCE on $\mathrm{PdCu}(110)$ is kinetically and thermodynamically favorable. These experimental findings are consistent with the theoretical results of Barbosa and Sautet (67). 


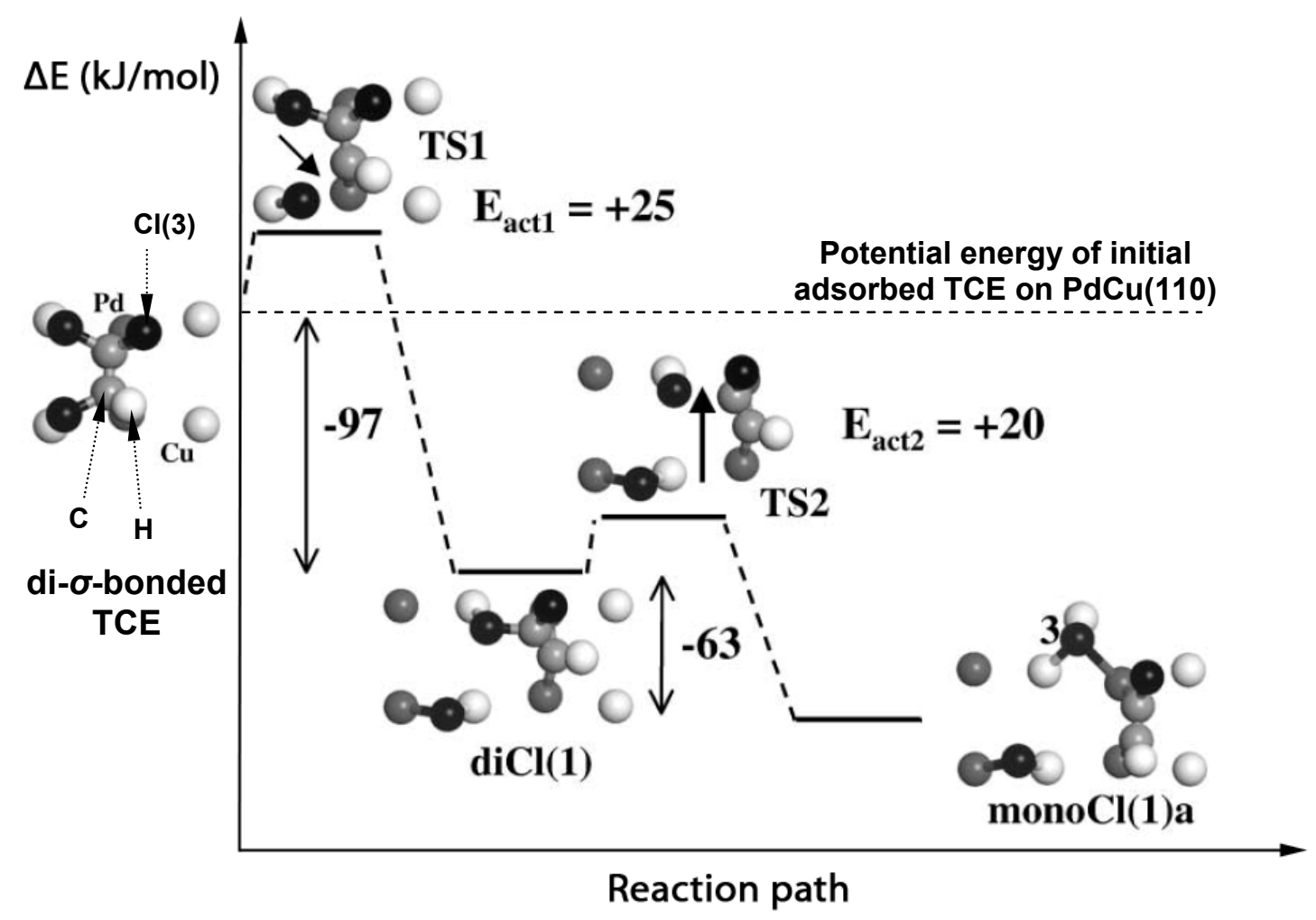

Figure I-8. Dechlorination reaction path for the di- $\sigma$-bonded TCE on $\mathrm{PdCu}(110)$. Ts denotes the transition states. The bold arrows indicate the $\mathrm{C}-\mathrm{Cl}$ bond which is being cleaved (adapted from Barbosa and Sautet (67)).

He et al. (68) studied the adsorption and dissociation of iso-, cis-, and trans-DCE on the $\operatorname{Si}(111) 7 \times 7$ surface using vibrational electron energy loss spectroscopy (EELS) and thermal desorption spectrometry (TDS). They observed mono- $\sigma$-bonded chlorovinyl $(\mathrm{HC}=\dot{\mathrm{C}} \mathrm{HCl})$ adspecies in the adsorption of the isomers. Upon annealing to $450 \mathrm{~K}$, the adspecies underwent further dechlorination to either vinylene $(H \dot{C}=\dot{C} H)$ di- $\sigma$-bonded to the Si surface, or acetylene released from the surface. This observed pathway is remarkably similar to the hypothesized pathway of reductive $\beta$-elimination suggested by Arnold and Roberts (6). Also, the feasibility of the formation of vinylene on the $\operatorname{Si}(111) 7 \times 7$ surface was proven using ab initio density functional calculations with a $\mathrm{Si}_{9} \mathrm{H}_{12}$ cluster, showing that the adsorption energy of vinylene is much more thermodynamically favorable than those of both the di- $\sigma$-bonded cycloaddition products and the mono- $\sigma$-bonded chlorovinyl $(\mathrm{H} \dot{\mathrm{C}}=\dot{\mathrm{C}} \mathrm{HCl})$ adspecies. 
Another investigation of dissociative adsorption focused on NO bond breaking on metal surfaces. Adsorption and dissociation of NO on metal surfaces are of considerable scientific and technological interest in relation to air pollution problems caused by car exhaust (66, 71). Loffreda et al. (71) investigated NO bond breaking on $\operatorname{Pd}(100), \operatorname{Rh}(100)$ and $\operatorname{Pd}_{3} \mathrm{Mn}(100)$ using periodic density functional theory to understand the electronic structure factors responsible for the remarkable activity of Rh for NO activation. The authors constructed a two-dimensional potential energy surface to intuit the pathway of NO dissociation by fixing the $x$ coordinates of both the $\mathrm{N}$ and $\mathrm{O}$ atoms but relaxing the other coordinates, including the metal atoms. Figure I-9 shows the most stable adsorption sites of NO on the Pd(100) surface, bridge and hollow sites (Figure I-9 (a) and (b)). NO is vertically adsorbed on the $\operatorname{Pd}(100)$ surface via the $\mathrm{N}$ atom. The potential energy surface indicates three possible pathways of NO dissociation on the $\mathrm{Pd}(100)$ surface: (I), (II) and (III), among which the second pathway, from the $\mathrm{H}$ site to the $\mathrm{H}-\mathrm{H}$ site, appears to be the most energetically favorable dissociation pathway (Figure I-9 (f)) because the H-H site is the global minimum with $\mathrm{N}$ and $\mathrm{O}$ atoms in neighboring hollow sites. In addition, the diffusion transition state, $\mathrm{T}_{4}$, can be readily formed because it is located between two low energy $\mathrm{H}$ and $\mathrm{B}$ sites. They concluded that a precursor for the NO dissociation is a NO structure "lying-down" horizontally on the metal surfaces. For the $\operatorname{Pd}(100)$ surface, the rotation of the NO molecule from the first stable vertical configuration to the horizontal one involves a large energy destabilization, while rotation on the $\mathrm{Rh}(100)$ surface requires a very small energy change. Interestingly, no stable horizontal species is achievable on the $\operatorname{Pd}(100)$ surface, whereas the horizontal structure is a stable species on the $\mathrm{Rh}(100)$. These factors are responsible for the higher activity of Rh for NO activation than $\operatorname{Pd}(100)$ and $\operatorname{Pd}_{3} \mathrm{Mn}(100)$. 


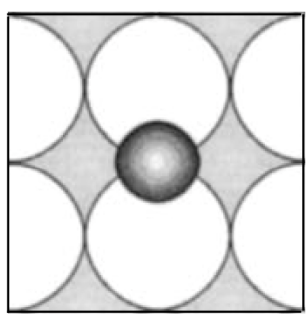

(a) B: bridge

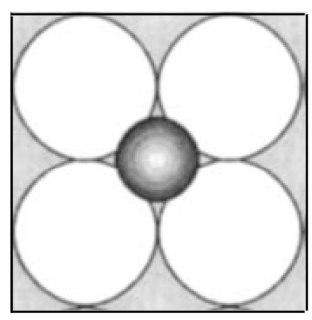

(b) H: hollow

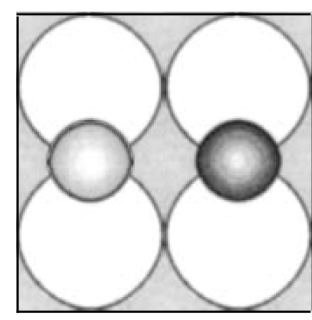

(c) B-B: bridge-bridge

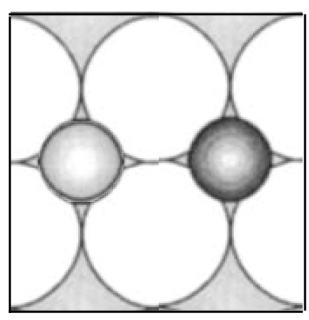

(d) $\mathrm{H}-\mathrm{H}$ : hollow-hollow
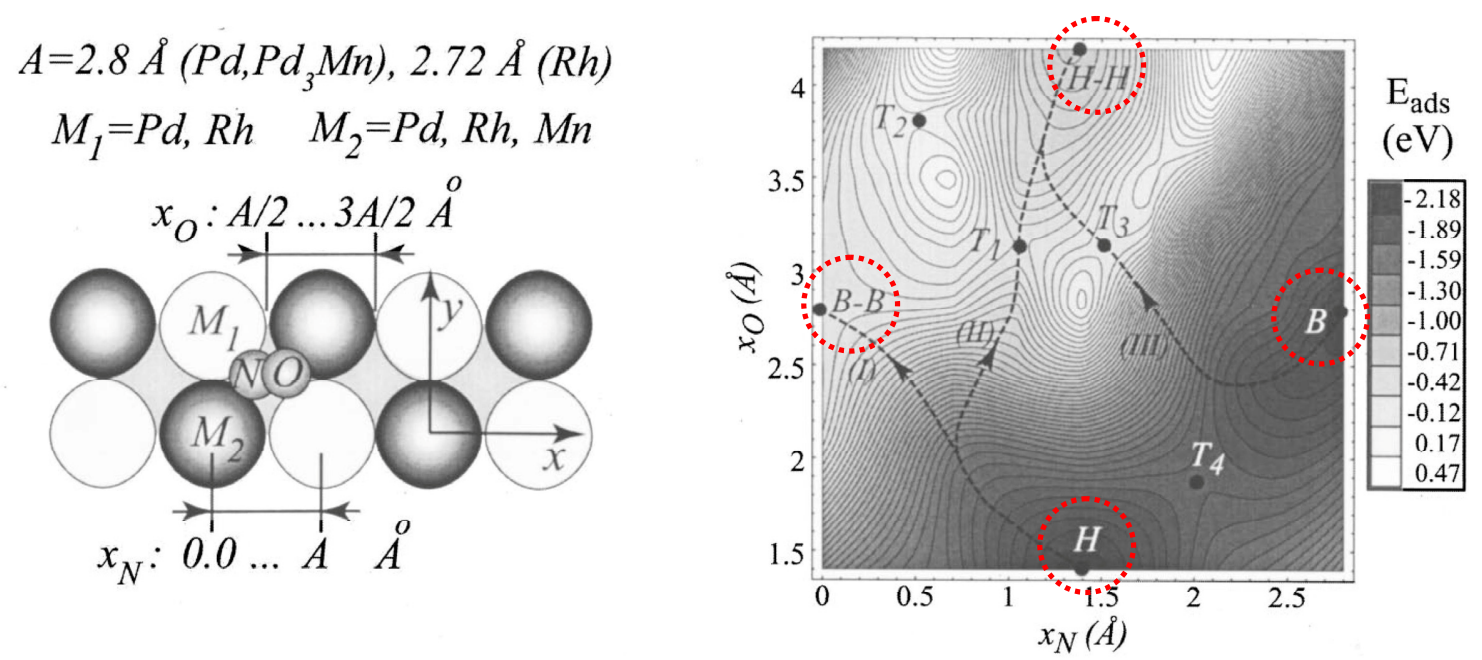

(e) Site dimensions

(f) Reaction path for NO dissociation and diffusion

Figure I-9. Two-dimensional potential energy surface for $\mathrm{NO}$ dissociation on the $\mathrm{Pd}$ surface; (a) and (b): the most stable adsorption sites; (c) and (d): light gray sphere represents the $\mathrm{N}$ atom and dark gray sphere represents the $\mathrm{O}$ atom; (e): site dimensions; (f) reaction path for $\mathrm{NO}$ dissociation and diffusion. $\mathrm{T}_{1}$ and $\mathrm{T}_{3}$ : dissociation transition states, $\mathrm{T}_{2}$ and $\mathrm{T}_{4}$ : diffusion transition states (adapted from Loffreda et al. (71)).

Brown and King (66) reviewed the studies of NO dissociation on various metal surfaces (Co, Ni, Rh, Ru and Ir). They concluded that NO dissociation depends strongly on coverage, usually with inhibited dissociation at high coverages. The dissociative adsorption of $\mathrm{NO}$ on the $\mathrm{Ni}(100)$ surface, for example, is no longer energetically favorable at high coverage (above 0.16 ML (mono-layer)) due to repulsive interactions between the adsorbed $\mathrm{N}$ and $\mathrm{O}$ atoms. At high coverage, a lack of vacant sites prevents the NO molecule from undergoing a two-step dissociation in which it first adsorbs onto the surface, and then reorients to allow the $\mathrm{O}$ atom to come into contact with the surface. In general, molecular adsorption of NO occurs at low temperature and dissociative 
adsorption of $\mathrm{NO}$ at higher temperature; however, the effect of temperature on NO dissociation significantly depends on the initial coverage of NO. Dissociation of NO on the Co surface, for instance, occurs at $150 \mathrm{~K}$ at low NO coverages, but for surfaces saturated with NO, the NO monomer is stable up to $250 \mathrm{~K}$.

\subsubsection{Analysis of bond cleavage and formation}

\section{Comparison of bond length}

The chemisorption and dissociation of adsorbates on metal surfaces involve both bond cleavage and formation. In order to elucidate the bond activation, several types of analyses are performed on: 1) bond length and frequency, 2) rebonding energy, 3) distortion energy of transition states, and 4) Mulliken overlap populations. The comparison of bond length is the simplest of these analyses. The bond activation for dissociation can be represented by the bond elongation $(63,67,71,72)$, as well as by the decrease of the intensity of a bond stretching vibration $(49,65,71)$. Barbosa and Sautet (67) noted that an early transition state shows a moderate elongation, from 0.13 to $0.37 \%$ of each initial state, while a late transition state shows a strongly elongated bond. The $\mathrm{C}-\mathrm{Cl}$ bond activation in TCE on the $\mathrm{PdCu}(110)$ surface is an example of an early transition state and the $\mathrm{C}-\mathrm{H}$ dissociation reaction on metal surfaces is an example of a late transition state.

\section{Vibrational frequency}

Bonding strength can also be measured by analyzing the bond vibration frequency. Because atoms in a molecule are constantly moving around average positions and not fixed in a single position, the molecule produces an oscillating electric field. Molecules absorb infrared radiation only when the frequency of the oscillating electronic field is the same as that of infrared radiation. The vibrational frequency can be measured using infrared (IR) spectroscopy or electron loss spectroscopy (EELS), as well as calculated using ab initio calculation. The frequency decreases with increasing atomic weight and increases with increasing bond energy (73). The frequency of single bond stretch, for example, is usually greater than $3000 \mathrm{~cm}^{-1}$ (e.g. $\mathrm{C}-\mathrm{H}, \mathrm{O}-\mathrm{H}$, and $\mathrm{N}-\mathrm{H}$ ), the frequency of double bond stretch is between $1500 \sim 2000 \mathrm{~cm}^{-1}$ and the frequency of triple bond stretch 
is between $2000 \sim 2500 \mathrm{~cm}^{-1}$. Specifically, the frequencies of the $\mathrm{C}-\mathrm{C}, \mathrm{C}=\mathrm{C}$ and $\mathrm{C} \equiv \mathrm{C}$ bonds are 1200, 1660 and $2200 \mathrm{~cm}^{-1}$, respectively (73). By comparing the vibration frequency of the gas-phase adsorbate with that of the adsorbed molecule, the changes in bonding characteristics can be interpreted.

\section{Rebonding energy}

The rebonding energy analysis suggested by (74) is meaningful to understanding transition states. Transition state potential energy, $E_{t s}$, can be written (Equation 6) ;

$$
E_{\text {ts }}=E_{\text {rebond }}+E_{\text {int }}
$$

where $E_{\text {rebond }}$ is the rebonding potential energy for the reaction products, and $E_{\text {int }}$ is the intra-molecular interaction among atoms in a molecule at the transition state. The rebonding energy characterizes the non-interacting reaction products, formed during the transition state $(67,74)$. This analysis is especially useful in understanding the late transition states, where the molecule is almost completely dissociated. NO dissociation on metal surfaces is a good example for the rebonding energy analysis of the late transition state. The low activation reaction barriers for $\mathrm{NO}$ dissociation can be explained by large rebonding energies; in other words, the high energy for the transition state is clearly due to a small atom-surface interaction $(71,74)$. Barbosa and Sautet (67) noted that the rebonding energy analysis also can be used for the early transition states of the $\mathrm{C}-\mathrm{Cl}$ bond cleavage of TCE, because the $\mathrm{Cl}$ atom is a common fragment in all transition states. The authors also suggested the rebonding energy of the $\mathrm{Cl}$ atom $\left(E_{\text {rebond }} \mathrm{Cl}\right)$ in the transition state be calculated as follows (Equation 7);

$$
E_{\text {rebond }} \mathrm{Cl}=E_{C l+\text { surf }}^{T s}-\left(E_{\text {surf }}+E_{C l}\right)
$$

where $E_{C l+s u r f}^{T s}$ is the energy of the chlorine fragment and the surface in the transition state; $E_{\text {surf }}$ is the energy of the surface; and $E_{C l}$ is the energy of the chlorine fragment in the gas-phase. It was concluded that, by contrast with NO dissociation, a low activation

energy barrier is associated with low $\mathrm{Cl}$ rebonding energy. This is on account of the early transition state of the $\mathrm{C}-\mathrm{Cl}$ dissociation, in which the less distorted geometry for the $\mathrm{C}-\mathrm{Cl}$ bond is responsible for the lower activation barrier. In support of this explanation, 
the authors performed another bond analysis, for the distortion energy of transition states, that indicates how modified the transition-state geometry is in relation to the initial state. The distortion energy of transition states $\left(E_{\text {distortion }}\right)$ can be written as follows (Equation 8);

$$
E_{\text {distortion }}=E_{\text {transition-state }}-E_{\text {reactant }}
$$

where $E_{\text {transition-state }}$ is the enegy of the molecule in the transition state and $E_{\text {reactant }}$ is the enegy of the ground state geometries calculated in the gas phase. The authors showed that the activation energy increases with the distortion energy, consistent with the correlation between the activation barrier energy and the rebonding energy for $\mathrm{C}-\mathrm{Cl}$ bond dissociation.

\section{Mulliken population analysis}

Mulliken overlap populations serve as a useful bonding index, allowing the measurement of the shared electron density between two atoms. Population analysis methods partition the electron density between the nuclei, which provides a way to calculate the atomic charge on each nucleus (75). The Mulliken population analysis permits a partitioning of the electrons among orbitals and atoms, and the overlap population can represent bond characteristics, in particularly bond strengths $(76,77)$. It should be noted that such an analysis provides only qualitative trends of bond strengths in systems with similar bond distances $(71,76)$. Loffreda et al. (71), for example, analyzed the overlap populations for $\mathrm{NO}$ dissociation on $\mathrm{Rh}(100), \operatorname{Pd}(100)$, and $\operatorname{Pd}_{3} \operatorname{Mn}(100)$ surfaces; results are shown in Figure I-10. Positive overlap populations indicate bond formation between $\mathrm{N}$ or $\mathrm{O}$ atoms and the surface, as well as the relative bond strengths among different surfaces (77); the bonds between $\mathrm{N}$ and $\mathrm{O}$ atoms and the $\mathrm{Rh}$ surface, for example, are stronger than those for the Pd surface. Moreover, the bonds between surface metal atoms are weakened as shown in the percentage changes relative to the bare surface as shown in Figure I-10. These results correspond to an increase of the atomic binding energy in the order $\mathrm{Pd}<\mathrm{Pd}_{3} \mathrm{Mn}<\mathrm{Rh}$. Correspondingly, $\mathrm{Rh}$ has the lowest reaction barrier for NO dissociation. 


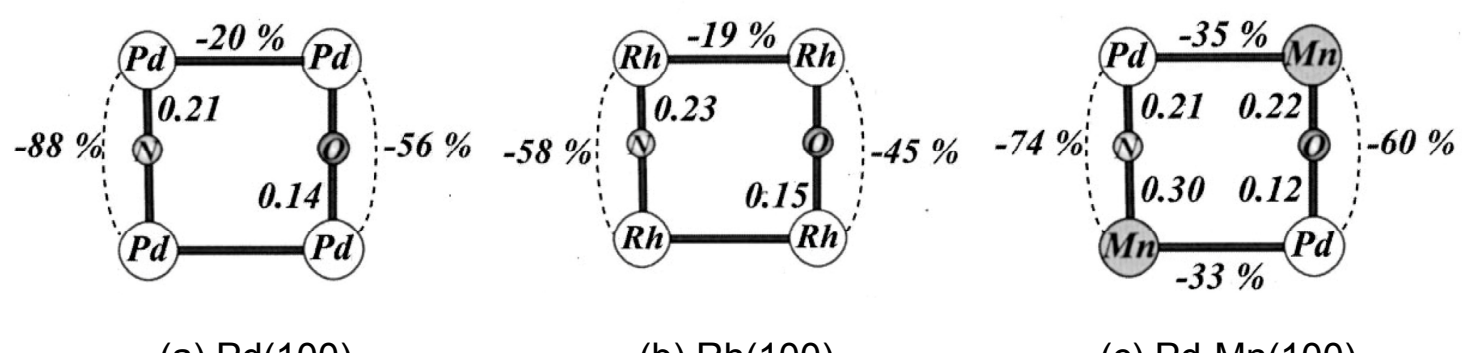
(a) $\operatorname{Pd}(100)$
(b) $\operatorname{Rh}(100)$
(c) $\operatorname{Pd}_{3} \mathrm{Mn}(100)$

Figure I-10. Mulliken overlap populations between atoms (the relative changes of the metal-metal bonds are shown as percentages) (adapted from Loffreda et al. (71)). 


\subsection{Summary of literature review and chapter overview}

Literature review was performed to understand degradation pathways of chloroethenes, adsorption of chloroethenes on metal surfaces, and dissociation of chloroethenes on metal surfaces. Abiotic reductive dechlorination of chloroethenes by transition metals occurs through $\beta$-elimination, hydrogenolysis, $\alpha$-elimination, and hydrogenation. Among them, reductive $\beta$-elimination by which two chlorines are simultaneously detached from the $\mathrm{C}=\mathrm{C}$ backbones of chloroethenes, is the most strongly kinetically favored mechanism. Reduction of PCE, TCE, and cis-DCE by reductive $\beta$ elimination produces dichloroacetylene, chloroacetylene, and acetylene, respectively. Hypothesized mechanisms for PCE degradation with iron particles by Arnold and Roberts (6) suggest that PCE is adsorbed on the iron surface with $\pi$-bonding and proceeds to form a di- $\sigma$-bonded surface-adsorbed species. PCE is then reduced through reductive $\beta$-elimination and hydrogenolysis (i.e., one chlorine is replaced by a hydrogen atom).

In review of adsorption of chloroethenes on metal surfaces, various experimental and theoretical studies reported di- $\sigma$ - and $\pi$-bond formation between chlorethenes and metal surfaces. $s p^{2}$ bonding of the carbon atoms of chloroethenes is modified by adsorption to resemble an alkane-like $s p^{3}$ bonding, $s p^{2}-s p^{3}$ hybridization. The most favorable adsorption sites for small molecules including carbon or hydrogen on $\mathrm{Fe}(110)$ and $\mathrm{Fe}(100)$ were reviewed in order to screen possible adsorption sites for chloroethenes on the $\mathrm{Fe}(110)$ surface. Chloroethene adsorption and dissociation may be significantly affected by the presence of water molecules. Water molecules markedly alter the adsorbed configuration of TCE on clay mineral. Reductive dechlorination of TCE is also more likely to occur when water is present than when it is not.

In review of dissociation of chloroethenes on metal surfaces, the investigation of dissociation mechanisms need to identify the most favorable adsorption and product configurations. Once the stationary geometries are found, the transition states between reactants and products are then located to calculate activation energies. Dechlorination of TCE on transition metals is kinetically and thermodynamically favorable. Activation energies of TCE intermediates are relatively small, less than $40 \mathrm{~kJ} / \mathrm{mol}$. The first $\mathrm{C}-\mathrm{Cl}$ bond cleavage of TCE is more favored in the $\mathrm{CCl}_{2}$ group rather than in the $\mathrm{CHCl}$ group. 
The following chapters consist of construction of iron surface model (Chapter II), adsorption of chloroethenes on $\mathrm{Fe}(110)$ (Chapter III), dissociation of chloroethenes on $\mathrm{Fe}(110)$ (Chapter IV), and conclusions and future work (Chapter V). Chapter II shows how a proper iron surface model is constructed to investigate adsorption and dissociation of chloroethenes. Details of computational methods are introduced and physical properties of bulk iron are verified utilizing these methods. Based on verified computational methods, a periodic three-layer slab model of $\mathrm{Fe}(110)$ surface is constructed. Chapter III presents results of adsorption of chloroethenes on the $\mathrm{Fe}(110)$ surface. The magnitude order of adsorption energies among chloroethenes is determined while taking into consideration solvent effects. Details of adsorption configurations and geometries of chloroethenes are presented. Electronic properties of the adsorbed chloroethenes are also analyzed to better understand the interaction between chloroethenes and the iron surface using the projected density of states (PDOS), the highest occupied molecular orbitals (HOMOs), and the lowest unoccupied molecular orbitals (LUMOs). Chapter IV presents results of dissociation of chloroethenes on the $\mathrm{Fe}(110)$ surface. Activation energies for dechlorination of chloroethenes are computed for reductive $\beta$-elimination pathways. The activation energies are converted to dechlorination reaction rate constants using an Arrhenius equation. The reaction rate constants of chloroethenes at their reaction rate-limiting steps are ultimately compared to determine the relative reactivity. The PDOS and charge density of transition states of chloroethenes are analyzed to understand the electronic properties of dissociated chloroethenes. Lastly, Chapter V concludes this study and suggests future work based on the limitations of this study. 


\section{References}

(1) Doherty, R. E., A history of the production and use of carbon tetrachloride, tetrachloroethylene, trichloroethylene and 1,1,1-trichloroethane in the United States: Part 1 - Historical background; Carbon tetrachloride and tetrachloroethylene. Environ. Forensics 2000, 1 (2), 69-81.

(2) Doherty, R. E., A history of the production and use of carbon tetrachloride, tetrachloroethylene, trichloroethylene and 1,1,1-trichloroethane in the United States: Part 2 - Trichloroethylene and 1,1,1-trichloroethane. Environ. Forensics 2000, 1 (2), 83-93.

(3) 11th Report on Carcinogens; Dept. of Health and Human Services, National Toxicology Program (NTP): 2005.

(4) Parker, B. L.; Gillham, R. W.; Cherry, J. A., Diffusive disappearance of immisciblephase organic liquids in fractured geologic media. Ground Water 1994, 32 (5), 805 820.

(5) Aggarwal, V.; Li, H.; Boyd, S. A.; Teppen, B. J., Enhanced sorption of trichloroethene by smectite clay exchanged with Cs+. Environ. Sci. Technol. 2006, 40 (3), 894-899.

(6) Arnold, W. A.; Roberts, A. L., Pathways and kinetics of chlorinated ethylene and chlorinated acetylene reaction with $\mathrm{Fe}(\mathrm{O})$ particles. Environ. Sci. Technol. 2000, 34 (9), 1794-1805.

(7) Orth, W. S.; Gillham, R. W., Dechlorination of trichloroethene in aqueous solution using Fe-O. Environ. Sci. Technol. 1996, 30 (1), 66-71.

(8) Henderson, A. D.; Demond, A. H., Long-term performance of zero-valent iron permeable reactive barriers: A critical review. Environ. Eng. Sci. 2007, 24 (4), 401423.

(9) Matheson, L. J.; Tratnyek, P. G., Reductive dehalogenation of chlorinated methanes by iron metal. Environ. Sci. Technol. 1994, 28 (12), 2045-2053.

(10) Arnold, W. A.; Roberts, A. L., Pathways of chlorinated ethylene and chlorinated acetylene reaction with $\mathrm{Zn}(0)$. Environ. Sci. Technol. 1998, 32 (19), 3017-3025.

(11) Johnson, T. L.; Scherer, M. M.; Tratnyek, P. G., Kinetics of halogenated organic compound degradation by iron metal. Environ. Sci. Technol. 1996, 30 (8), 26342640.

(12) Scherer, M. M.; Balko, B. A.; Gallagher, D. A.; Tratnyek, P. G., Correlation analysis of rate constants for dechlorination by zero-valent iron. Environ. Sci. Technol. 1998, 32 (19), 3026-3033.

(13) Lee, W. J.; Batchelor, B., Abiotic reductive dechlorination of chlorinated ethylenes by iron-bearing phyllosilicates. Chemosphere 2004, 56 (10), 999-1009.

(14) Lee, W.; Batchelor, B., Abiotic reductive dechlorination of chlorinated ethylenes by iron-bearing soil minerals. 1. Pyrite and magnetite. Environ. Sci. Technol. 2002, 36 (23), 5147-5154.

(15) Lee, W.; Batchelor, B., Abiotic, reductive dechlorination of chlorinated ethylenes by iron-bearing soil minerals. 2. Green rust. Environ. Sci. Technol. 2002, 36 (24), 5348-5354. 
(16) Lowry, G. V.; Reinhard, M., Hydrodehalogenation of 1-to 3-carbon halogenated organic compounds in water using a palladium catalyst and hydrogen gas. Environ. Sci. Technol. 1999, 33 (11), 1905-1910.

(17) Butler, E. C.; Hayes, K. F., Kinetics of the transformation of trichloroethylene and tetrachloroethylene by iron sulfide. Environ. Sci. Technol. 1999, 33 (12), 2021-2027.

(18) Burris, D. R.; Campbell, T. J.; Manoranjan, V. S., Sorption of trichloroethylene and tetrachloroethylene in a batch reactive metallic iron-water system. Environ. Sci. Technol. 1995, 29 (11), 2850-2855.

(19) Liu, Y. Q.; Majetich, S. A.; Tilton, R. D.; Sholl, D. S.; Lowry, G. V., TCE dechlorination rates, pathways, and efficiency of nanoscale iron particles with different properties. Environ. Sci. Technol. 2005, 39 (5), 1338-1345.

(20) Roberts, A. L.; Totten, L. A.; Arnold, W. A.; Burris, D. R.; Campbell, T. J., Reductive elimination of chlorinated ethylenes by zero valent metals. Environ. Sci. Technol. 1996, 30 (8), 2654-2659.

(21) Hara, J.; Ito, H.; Suto, K.; Inoue, C.; Chida, T., Kinetics of trichloroethene dechlorination with iron powder. Water Research 2005, 39 (6), 1165-1173.

(22) He, J. Z.; Sung, Y.; Dollhopf, M. E.; Fathepure, B. Z.; Tiedje, J. M.; Loffler, F. E., Acetate versus hydrogen as direct electron donors to stimulate the microbial reductive dechlorination process at chloroethene-contaminated sites. Environ. Sci. Technol. 2002, 36 (18), 3945-3952.

(23) Maymó-Gatell, X.; Chien, Y. T.; Gossett, J. M.; Zinder, S. H., Isolation of a bacterium that reductively dechlorinates tetrachloroethene to ethene. Science 1997, 276 (5318), 1568-1571.

(24) Wild, A.; Hermann, R.; Leisinger, T., Isolation of an anaerobic bacterium which reductively dechlorinates tetrachloroethene and trichloroethene. Biodegradation 1997, 7 (6), 507-511.

(25) Neumann, A.; Wohlfarth, G.; Diekert, G., Purification and characterization of tetrachloroethene reductive dehalogenase from Dehalospirillum multivorans. J. Biol. Chem. 1996, 271 (28), 16515-16519.

(26) Arcangeli, J. P.; Arvin, E.; Mejlhede, M.; Lauritsen, F. R., Biodegradation of cis1,2-dichloro-ethylene at low concentrations with methane-oxidizing bacteria in a biofilm reactor. Water Research 1996, 30 (8), 1885-1893.

(27) Verce, M. F.; Ulrich, R. L.; Freedman, D. L., Characterization of an isolate that uses vinyl chloride as a growth substrate under aerobic conditions. Appl. Environ. Microb. 2000, 66 (8), 3535-3542.

(28) Fathepure, B. Z.; Elango, V. K.; Singh, H.; Bruner, M. A., Bioaugmentation potential of a vinyl chloride-assimilating Mycobacterium sp., isolated from a chloroethene-contaminated aquifer. FEMS Microbiol. Lett. 2005, 248 (2), 227-234.

(29) Bradley, P. M.; Chapelle, F. H., Aerobic microbial mineralization of dichloroethene as sole carbon substrate. Environ. Sci. Technol. 2000, 34 (1), 221-223.

(30) Nakajima, T.; Uchiyama, H.; Yagi, O.; Nakahara, T., Novel metabolite of trichloroethylene in a methanotrophic bacterium, Methylocystis Sp M, and hypothetical degradation pathway. Biosci. Biotech. Bioch. 1992, 56 (3), 486-489.

(31) Kriegman-King, M. R.; Reinhard, M., Abiotic transformation of carbontetrachloride in the presence of sulfide and mineral surfaces. Abstr. Pap. Am. Chem. S. 1992, 203, 83-ENVR. 
(32) Doong, R. A.; Wu, S. C., Reductive dechlorination of chlorinated hydrocarbons in aqueous-solutions containing ferrous and sulfide ions. Chemosphere 1992, 24 (8), 1063-1075.

(33) Keum, Y. S.; Li, Q. X., Reductive debromination of polybrominated diphenyl ethers by zerovalent iron. Environ. Sci. Technol. 2005, 39 (7), 2280-2286.

(34) Sayles, G. D.; You, G. R.; Wang, M. X.; Kupferle, M. J., DDT, DDD, and DDE dechlorination by zero-valent iron. Environ. Sci. Technol. 1997, 31 (12), 3448-3454.

(35) Chuang, F. W.; Larson, R. A.; Wessman, M. S., Zero-valent iron-promoted dechlorination of polychlorinated-biphenyls. Environ. Sci. Technol. 1995, 29 (9), 2460-2463.

(36) O'Hannesin, S. F.; Gillham, R. W., Long-term performance of an in situ "iron wall" for remediation of VOCs. Ground Water 1998, 36 (1), 164-170.

(37) Reardon, E. J., Anaerobic corrosion of granular iron - measurement and interpretation of hydrogen evolution rates. Environ. Sci. Technol. 1995, 29 (12), 2936-2945.

(38) Gotpagar, J.; Lyuksyutov, S.; Cohn, R.; Grulke, E.; Bhattacharyya, D., Reductive dehalogenation of trichloroethylene with zero-valent iron: Surface profiling microscopy and rate enhancement studies. Langmuir 1999, 15 (24), 8412-8420.

(39) Barbosa, L.; Loffreda, D.; Sautet, P., Chemisorption of trichloroethene on the PdCu alloy (110) surface: A periodical density functional study. Langmuir 2002, 18 (7), 2625-2635.

(40) Bloxham, L. H.; Haq, S.; Mitchell, C.; Raval, R., Dynamical catalytic responses in the reaction of trans-1,2-dichloroethene on Pd(110). Surf. Sci. 2001, 489 (1-3), 1-19.

(41) Yang, M. X.; Kash, P. W.; Sun, D. H.; Flynn, G. W.; Bent, B. E.; Holbrook, M. T.; Bare, S. R.; Fischer, D. A.; Gland, J. L., Chemistry of chloroethylenes on $\mathrm{Cu}(100)$ : Bonding and reactions. Surf. Sci. 1997, 380 (2-3), 151-164.

(42) Valcárcel, A.; Clotet, A.; Ricart, J. M.; Illas, F., Comparative theoretical study of the structure and bonding of propyne on the $\operatorname{Pt}(111)$ and $\operatorname{Pd}(111)$ surfaces. Chem. Phys. 2005, 309, 33-39.

(43) Jiang, D. E.; Carter, E. A., Adsorption and diffusion energetics of hydrogen atoms on Fe(110) from first principles. Surf. Sci. 2003, 547 (1-2), 85-98.

(44) Yanagisawa, S.; Tsuneda, T.; Hirao, K.; Matsuzaki, Y., Theoretical investigation of adsorption of organic molecules onto Fe(110) surface. J. Mol. Struc.-Theochem 2005, $716(1-3), 45-60$.

(45) Sorescu, D. C.; Thompson, D. L.; Hurley, M. M.; Chabalowski, C. F., Firstprinciples calculations of the adsorption, diffusion, and dissociation of a $\mathrm{CO}$ molecule on the Fe(100) surface. Phys. Rev. B 2002, 66 (3).

(46) Valcárcel, A.; Gil, A.; Ricart, J. M.; Clotet, A., Theoretical study of propene adsorbed on sulphated Pt(111). Chem. Phys. Lett. 2004, 399 (4-6), 295-299.

(47) Hartnig, C.; Spohr, E., The role of water in the initial steps of methanol oxidation on Pt(111). Chem. Phys. 2005, 319 (1-3), 185-191.

(48) Lake, R. F.; Thompson, H., Photoelectron spectra of halogenated ethylenes. P. Roy. Soc. Lond. A Mat. 1970, 315 (1522), 323-\&.

(49) Jugnet, Y.; Prakash, N. S.; Bertolini, J. C.; Raval, R., HREELS investigations of trans-dichloroethene adsorption and chemical reaction on $\mathrm{Cu}(110)$. Catal. Lett. 1998, 56 (1), 17-21. 
(50) Cassuto, A.; Hugenschmidt, M. B.; Parent, P.; Laffon, C.; Tourillon, H. G., A NEXAFS and UPS study of the adsorption of tetrachloroethylene, trichloroethylene, iso-dichloroethylene, cis-dichloroethylene, and trans-dichloroethylene on platinum surfaces at 95 K: multilayers and monolayers. Surf. Sci. 1994, 310 (1-3), 390-398.

(51) Grassian, V. H.; Pimentel, G. C., The structures of cis- and trans-dichloroethenes adsorbed on $\mathrm{Pt}(111)$. J. Chem. Phys. 1988, 88 (7), 4478-4483.

(52) Jiang, D. E.; Carter, E. A., Carbon atom adsorption on and diffusion into Fe(110) and $\mathrm{Fe}(100)$ from first principles. Phys. Rev. B 2005, 71 (4).

(53) Satoh, S.; Fujimoto, H.; Kobayashi, H., Theoretical study of NH3 adsorption on $\mathrm{Fe}(110)$ and $\mathrm{Fe}(111)$ surfaces. J. Phys. Chem. B 2006, 110 (10), 4846-4852.

(54) Jiang, D. E.; Carter, E. A., First principles study of H2S adsorption and dissociation on Fe(110). Surf. Sci. 2005, 583 (1), 60-68.

(55) Jiang, D. E.; Carter, E. A., Adsorption and dissociation of $\mathrm{CO}$ on $\mathrm{Fe}(110)$ from first principles. Surf. Sci. 2004, 570 (3), 167-177.

(56) Eder, M.; Terakura, K.; Hafner, J., Initial stages of oxidation of (100) and (110) surfaces of iron caused by water. Phys. Rev. B 2001, 64 (11).

(57) Zhou, H.; Tamura, H.; Takami, S.; Kubo, M.; Zhanpeisov, N.; Miyamoto, A., Adsorption properties of $\mathrm{CH}_{3} \mathrm{OH}$ on $\mathrm{Al}$ (111) and $\mathrm{Fe}$ (100) surfaces: A periodic first-principles investigation. Jpn. J. Appl. Phys. 1 2000, 39 (7B), 4275-4278.

(58) Benndorf, C.; Madey, T. E.; Johnson, A. L., NH3 adsorption and dissociation on a stepped Fe(S)(100) surface. Surf. Sci. 1987, 187 (2-3), 434-444.

(59) Desai, S. K.; Pallassana, V.; Neurock, M., A periodic density functional theory analysis of the effect of water molecules on deprotonation of acetic acid over Pd(III). J. Phys. Chem. B 2001, 105 (38), 9171-9182.

(60) Neurock, M.; Wasileski, S. A.; Mei, D., From first principles to catalytic performance: tracking molecular transformations. Chem. Eng. Sci. 2004, 59 (22-23), 4703-4714.

(61) Okamoto, Y.; Sugino, O.; Mochizuki, Y.; Ikeshoji, T.; Morikawa, Y., Comparative study of dehydrogenation at $\mathrm{Pt}(111) /$ water and $\mathrm{Pt}(111)$ /vacuum of methanol interfaces. Chem. Phys. Lett. 2003, 377 (1-2), 236-242.

(62) Schravendijk, P.; van der Vegt, N.; Delle Site, L.; Kremer, K., Dual-scale modeling of benzene adsorption onto $\mathrm{Ni}(111)$ and $\mathrm{Au}(111)$ surfaces in explicit water. Chemphyschem 2005, 6 (9), 1866-1871.

(63) Teppen, B. J.; Yu, C. H.; Newton, S. Q.; Miller, D. M.; Schafer, L., Quantum molecular dynamics simulations regarding the dechlorination of trichloro ethene in the interlayer space of the $2: 1$ clay mineral nontronite. J. Phys. Chem. A 2002, 106 (22), 5498-5503.

(64) Hung, W. H.; Schwartz, J.; Bernasek, S. L., Sequential oxidation of Fe(100) by water-adsorption - formation of an ordered hydroxylated surface. Surf. Sci. 1991, $248(3), 332-342$.

(65) Jugnet, Y.; Bertolini, J. C.; Barbosa, L.; Sautet, P., Vibrational identification of the surface reaction intermediates for the dehalogenation of trichloroethene on $\operatorname{PdCu}(110)$ alloy. Surf. Sci. 2002, 505 (1-3), 153-162.

(66) Brown, W. A.; King, D. A., NO chemisorption and reactions on metal surfaces: A new perspective. J. Phys. Chem. B 2000, 104 (12), 2578-2595. 
(67) Barbosa, L.; Sautet, P., Trichloroethene dechlorination reactions on the $\mathrm{PdCu}(110)$ alloy surface: A periodical density functional theory study of the mechanism. $J$. Catal. 2002, 207 (1), 127-138.

(68) He, Z. H.; Li, Q.; Leung, K. T., Isomeric effects on room-temperature chemisorption and thermal evolution of iso-, cis- and trans-dichloroethylene on Si(111)7x7. Surf. Sci. 2006, 600 (3), 514-526.

(69) Yang, M. X.; Sarkar, S.; Bent, B. E.; Bare, S. R.; Holbrook, M. T., Degradation of multiply-chlorinated hydrocarbons on $\mathrm{Cu}(100)$. Langmuir 1997, 13 (2), 229-242.

(70) Yang, M. X.; Eng, J.; Kash, P. W.; Flynn, G. W.; Bent, B. E.; Holbrook, M. T.; Bare, S. R.; Gland, J. L.; Fischer, D. A., Generation and reaction of vinyl groups on a $\mathrm{Cu}(100)$ surface. J. Phys. Chem. 1996, 100 (30), 12431-12439.

(71) Loffreda, D.; Delbecq, F.; Simon, D.; Sautet, P., Breaking the NO bond on Rh, Pd, and Pd3Mn alloy (100) surfaces: A quantum chemical comparison of reaction paths. J. Chem. Phys. 2001, 115 (17), 8101-8111.

(72) Zhang, N. L.; Blowers, P.; Farrell, J., Ab initio study of carbon-chlorine bond cleavage in carbon tetrachloride. Environ. Sci. Technol. 2005, 39 (2), 612-617.

(73) Wade, L. G., Organic Chemistry. Prentice Hall: 2002; p Chapter 12.

(74) Hammer, B., Adsorption, diffusion, and dissociation of NO, N and $\mathrm{O}$ on flat and stepped Ru(0001). Surf. Sci. 2000, 459 (3), 323-348.

(75) Leach, A. R., Molecular Modelling. Principles and Application. 2nd ed.; Prentice Hall: 2001; p 79.

(76) Glassey, W. V.; Hoffmann, R., A comparative study of Hamilton and overlap population methods for the analysis of chemical bonding. J. Chem. Phys. 2000, 113 (5), 1698-1704.

(77) Mulliken, R. S., Electronic population analysis on LCAO-MO molecular wave functions .1. J. Chem. Phys. 1955, 23 (10), 1833-1840. 


\section{CHAPTER II \\ CONSTRUCTION OF IRON SURFACE MODEL}

\section{Computational methods}

\subsection{Density functional theory (DFT) and generalized gradient approximation (GGA)}

All electronic structure calculations were performed using periodic density functional theory (DFT) and the ultrasoft Vanderbilt pseudopotential method (1) within the generalized gradient approximation (GGA) by Perdew, Burke, and Ernzerhof (PBE) (2) for the exchange-correlation functional as implemented in the Plane-Wave SelfConsistent Field (PWscf) code in the Quantum-ESPRESSO open-source distribution (3). The Monkhorst-Pack scheme (4) was used for the $k$-point sampling.

A brief explaination of the DFT method and the GGA-PBE method is summarized herein (5-7). DFT is a quantum mechanical method to investigate the electronic structure of molecules, solids and liquid. The theory has been successfully used to obtain thermochemical data, molecular structures, transition structure, force fields and frequencies, as well as activation barriers. While the traditional Hartree-Fock theory method is based on the complicated many-electron wavefunction to investigate the electronic structures, the main notion of density functional theory is that the energy of an electronic system can be expressed in terms of the electron density as the basic quantity instead of the many-body electronic wavefunction. The Kohn-Sham equation has served as a starting point for the DFT method. The total energy $\left(E_{T O T}\right)$ of an $n$-electron system can be expressed as

$$
E_{T O T}=E_{\text {Kinetic }}+E_{\text {Attraction }}+E_{\text {Coulomb }}+E_{\text {Exchange-Correlation }}
$$

where $E_{\text {kinetic }}$ represents the kinetic energy of $n$ noninteracting electrons with the same density as the actual system of interacting electrons; $E_{\text {Atraction }}$ represents the electron- 
nucleus attraction; $E_{\text {Coulomb }}$ represents the Coulomb interaction between the two charge distributions; $E_{\text {Exchange-Correlation }}$ (or $E_{X C}$ ) represents the exchange-correlation energy.

Although electrons in a molecular orbital exclude one another, the HF method does not consider the electron exclusion effect. However, DFT takes into account the electron exclusion effect using the term of $E_{X C}$ by creating a spherical hole (or no-fly zone) into which other electrons will not penetrate. The shape of the hole, $E_{X C}$, in principle can be expressed as a function of electron density. If an electronic system has $m$ electrons which obey the Pauli principle and repulse each other via the Coulomb potential, the wavefunction includes $3 m$ variables; however, the electronic density, no matter how large the system is, depends only on three variables $x, y$, and $z$. Consequently, the reduced number of the variables in DFT considerably reduces the computational effort compared to the traditional electronic structure theories.

However, the exact functionals for the exchange-correlation (XC) are not known except for the free electron gas. Thus, the exchange-correlation energy $E_{X C}$ as a functional of spin-up and -down electron densities at point $\mathbf{r}\left(n_{\uparrow}(\mathbf{r})\right.$ and $n_{\downarrow}(\mathbf{r})$, respectively) must be approximated (2). The most widely used approximations are the local-density approximation (LDA) and the generalized gradient approximation (GGA). The GGA creates the electron exclusion hole using electron density gradients to express nonuniform charge densities; however, the LDA approximates an inhomogeneous system using homogeneous electron gas with the same density at every point in the system. In general, the GGA improves upon the LDA in many aspects, especially for atomic energies and structural energy differences $(2,8$, 9). It is well known that the LDA overestimates the binding energy, and underestimates the bond length by $1-2 \%$, which results in the calculated equilibrium volume normally $3-6 \%$ less than experiment (10). However, the GGA tends to overestimate the bond length by about 1\% (11). 
The LDA is written as

$$
E_{X C}^{L D A}[n]=\int d^{3} r n \varepsilon_{X C}(n)
$$

where $n$ is homogeneous electron gas density and $\varepsilon_{X C}(n)$ is the exchange and correlation energy per particle. A straightforward generalization of the LDA including electron spin is the local spin density (LSD) approximation as follows (2).

$$
E_{X C}^{L S D}\left[n_{\uparrow}, n_{\downarrow}\right]=\int d^{3} r n \varepsilon_{X C}\left(n_{\uparrow}, n_{\downarrow}\right)
$$

where $n=n_{\uparrow}+n_{\downarrow}$, and $n_{\uparrow}$ and $n_{\downarrow}$ are spin-up and -down electron densities, respectively. The GGA is written as (2)

$$
E_{X C}^{G G A}\left[n_{\uparrow}, n_{\downarrow}\right]=\int d^{3} r f\left(n_{\uparrow}, n_{\downarrow}, \nabla n_{\uparrow}, \nabla n_{\downarrow}\right)
$$

Where $\nabla n_{\uparrow}$ and $\nabla n_{\downarrow}$ are spin-up and -down electron gradients, respectively. 


\subsection{Pseudopotential and basis set}

As iron is an electron-rich transition metal, the pseudo-potential method was used. If all electrons in the transition metal are included when performing a calculation, the computational cost will be huge. Significant developments in computational quantum chemistry have been achieved with the advent of valence electron calculations based on effective core potentials (ECP). The valence electrons of an atom are largely responsible for the chemical bonding, while the core electrons are little affected by the atomic environment (12). By replacing inactive core electrons with some averaged potential, the computational effort involved in the DFT calculation can be considerably reduced. In the case of iron atom, the eighteen inner shell electrons $\left(1 s^{2}, 2 s^{2}, 2 p^{6}, 3 s^{2}, 3 p^{6}\right)$ are treated as inactive core electrons that participate sparingly in chemical bond formation (13). The remaining eight electrons in the valance layer $\left(3 d^{6}, 4 s^{2}\right)$ do participate in bond formation. A brief explaination of a basis set is summarized herein. A basis set is a collection of vectors numerically representing orbitals of an atom. The minimal basis set contains one orbital vector for each atomic orbital in the atom. A drawback of the minimal basis set is that it lacks any flexibility to expand or shirk in the presence of other atoms in a molecule. However, by assigning two basis functions to each atomic orbital, the flexibility can be added to the atom, which is called a double-zeta $(\zeta)$ basis set. Gaussian 03 provides the Los Alamos LanL2DZ double-zeta $(\zeta)$ basis set in which the first row atoms are represented by Dunning/Huzinaga full double-zeta $(\zeta)$ basis sets, and the iron atom by the Los Alamos ECP plus double-zeta basis set (14). As eight electrons are in the valence layer of iron atom, total sixteen basis functions are considered in the calculation of iron atom with the double-zeta basis set. 


\subsection{Cluster model and periodic boundary condition (PBC) model}

Cluster model simulations have been performed to investigate the interactions between adsorbate molecules and metal surfaces. Although some of the results of the cluster simulations show better agreement with experimental data than results obtained from periodic slab calculations (15), it is recognized that the results from cluster calculations depend significantly on the cluster sizes and on the method used for terminating the cluster model $(16,17)$. Nevertheless, the cluster model calculation is useful to obtain good insights on the local interactions between a part or the whole of the adsorbate molecule and metal surface. Figure II-1 shows the Fe(110) surface. It is noted the electronic properties of the $\mathrm{Fe}$ atoms in the cluster are all different from one another. To effectively simulate the interaction between adsorbates and the cluster model surface, the cluster should be large enough to equip reasonably similar electronic properties near sites where adsorbates interact. However, quantum chemical calculations of electron-rich transition metal systems frequently encounter convergence problems and require large amounts of computational resources as the cluster size increases. So one must tradeoff cluster size for computational efficiency.
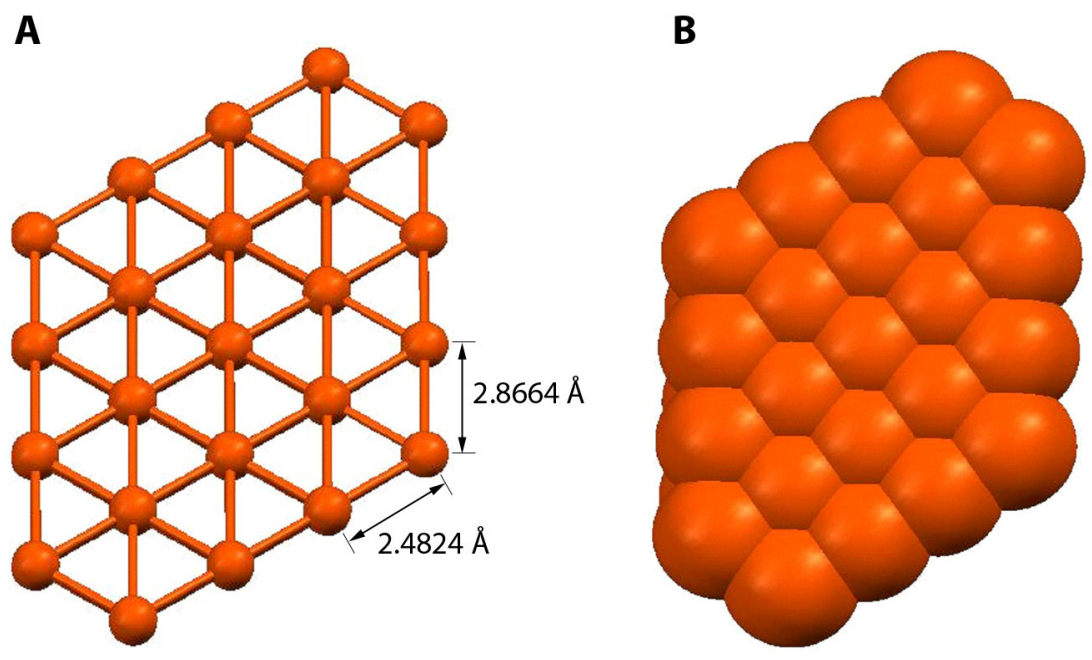

Figure II-1. Cluster model of Fe(110) surface. (A) Ball and stick model. (B) Spacefill model. 
Periodic boundary conditions (PBC) are particularly useful when simulating an effectively infinite surface system, such as metal or mineral surfaces. The cluster model reveals different electronic properties among atoms depending on different locations because atoms near the boundary of a cluster model have less neighbors than atoms inside, which is not a realistic condition. However, PBC model mimics natural characteristics of metal surfaces by replicating a cubical simulation box infinitely throughout $x^{-}, y^{-}$, and $z^{-}$- directions. If an atom is located at a position vector $\mathbf{r}$ in a simulation box, the identical atoms are assumed to be placed at $\mathbf{r}+(i \mathbf{x}+j \mathbf{y}+k \mathbf{z})$, where $i$, $j$, and $k$ are integer numbers varied from $-\infty$ to $+\infty$, and $\mathbf{x}, \mathbf{y}$, and $\mathbf{z}$ are the vectors corresponding to the edges of the box (18). Figure II-2 illustrates a two-dimensional version of such a periodic system of the $\mathrm{Fe}(110)$ surface. The rectangle box represents a unit cell of $\mathrm{Fe}(110)-\mathrm{p}(1 \times 1)$. Thus, the entire structure represents a $\mathrm{Fe}(110)-\mathrm{p}(3 \times 2)$ structure. This simulation box (i.e., supercell) is infinitely replicated throughout $x-$ and $y$ - directions. It is noted that the $\mathrm{Fe}$ atoms located at the bottom of the $\mathrm{p}(3 \times 2)$ structure are identical with those at the top line; the Fe atoms at the left-most are also identical with those at the right-most ones.

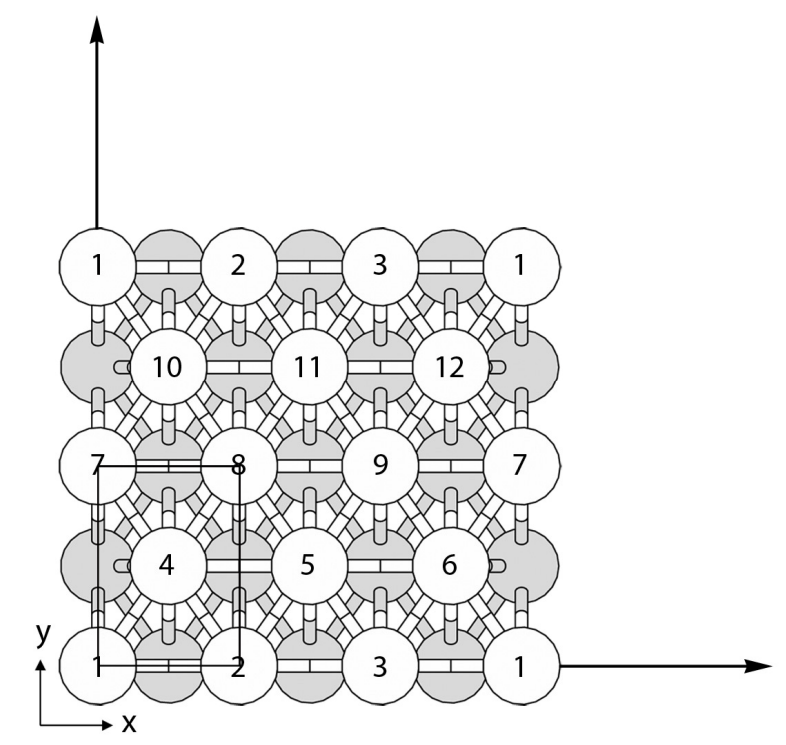

Figure II-2. $\mathrm{Fe}(110)-\mathrm{p}(3 \times 2)$ supercell used in a $\mathrm{PBC}$ model of the $\mathrm{Fe}(110)$ surface. Rectangle box represents a $\mathrm{p}(1 \times 1)$ unit cell. Numbers represent surface Fe atoms. Atoms in gray represent the second surface layer. Big and small arrows indicate the direction of replication of the supercell and coordinates, respectively. 


\section{Physical properties of bulk iron}

Physical properties of bulk iron were theoretically determined to compare with experimental values prior to constructing the periodic surface model. All electronic structure calculations were performed using periodic density functional theory and the ultrasoft Vanderbilt pseudopotential method (1) within the generalized gradient approximation (GGA) by Perdew, Burke, and Ernzerhof (PBE) (2) for the exchangecorrelation functional as implemented in the Plane-Wave Self-Consistent Field (PWscf) code in the Quantum-ESPRESSO open-source distribution (3). Kinetic-energy cutoffs for wavefunctions and for charge density and potential were $340.1 \mathrm{eV}$ (25 Ryd) and 2449.0 eV (180 Ryd), respectively; this converges the total energy to within $0.01 \mathrm{eV} /$ atom. The Monkhorst-Pack scheme (4) was used for the $k$-point sampling. Using a $10 \times 10 \times 10 k$ point mesh, the equilibrium lattice constant $\left(a_{0}\right)$ of ferromagnetic body-centered cubic (bcc) iron was determined using the Birch-Murnaghan equation of state (Equation 5) (19). The fit of the calculated total energies of bulk iron to the Birch-Murnaghan equation of state yields an equilibrium unit cell volume of $78.987 \mathrm{Bohr}^{3}$ and a bulk modulus of $0.01137 \mathrm{Ryd} / \mathrm{Bohr}^{3}$ (Figure II-3), which correspond to the equilibrium lattice constant $\left(a_{0}\right)$ of $2.8606 \AA$ and $167 \mathrm{GPa}$, respectively. Magnetic moment $(M)$ is also calculated as 2.45 $\mu_{\mathrm{B}}$. These results are in good agreement with experimental results (20) for ferromagnetic bcc iron: $a_{0}=2.8664 \AA, B=168 \mathrm{GPa}, M=2.22 \mu_{\mathrm{B}}$.

$$
E(V)=E_{0}+\frac{9 V_{0} B_{0}}{16}\left\{\left[\left(\frac{V_{0}}{V}\right)^{\frac{2}{3}}-1\right]^{3} B_{0}^{\prime}+\left[\left(\frac{V_{0}}{V}\right)^{\frac{2}{3}}-1\right]^{2}\left[6-4\left(\frac{V_{0}}{V}\right)^{\frac{2}{3}}\right]\right\}
$$

where $E$ : the total energy (Ryd), $V$ : the unit cell volume $\left(\mathrm{Bohr}^{3}\right), V_{0}$ : the equilibrium unit cell volume $\left(\mathrm{Bohr}^{3}\right), E_{0}$ : the minimum energy at $V_{0}$ (Ryd), $B_{0}$ : the bulk modulus $\left(\mathrm{Ryd} / \mathrm{Bohr}^{3}\right)$, and $B_{0}^{\prime}:$ the first derivative of the bulk modulus. 


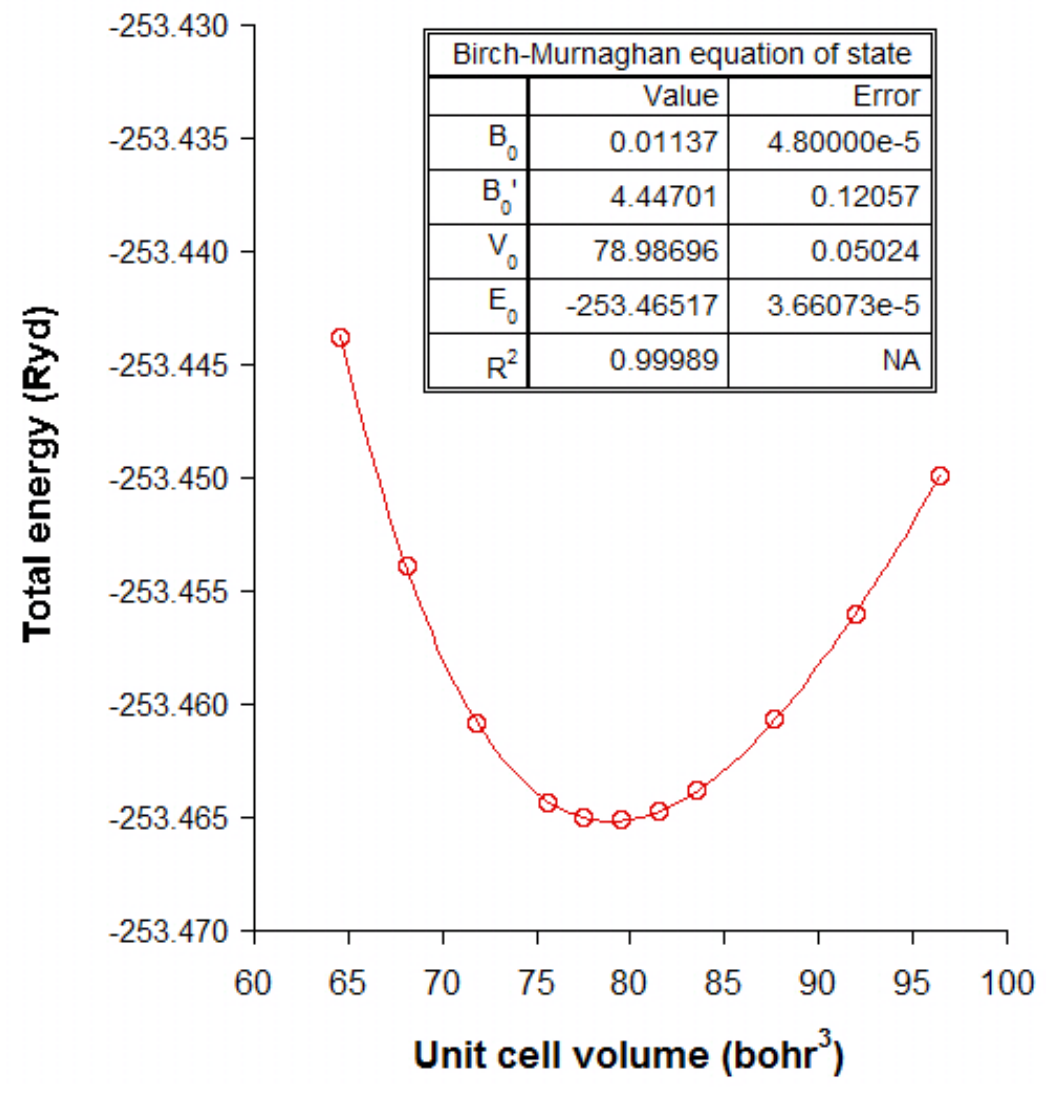

Figure II-3. Fitting of calculated total energies of bulk iron to the Birch-Murnaghan equation of state. 


\section{Construction of iron surface model}

\subsection{Different type of iron surface}

The unit cell (i.e., the smallest structure that repeats itself through crystals) of bulk iron is the body-centered cubic (bcc). The bcc system has one lattice point in the center of the unit cell and in the eight corner points (Figure II-4). The orientation of a surface of crystals is defined by the rules of the Miller Indices, $(h k l)$. The set of number of the Miller Indices quantify the intercepts on the $x-, y^{-}$, and $z^{-}$axes that identify a surface of crystals. The number zero in the Miller Indices indicates a surface is parallel to the axes represented by zero. The intercepts on the axes are represented by the reciprocals of fractional intercepts. The fractional intercepts are obtained by dividing coordinates by the respective cell dimension. The fractional intercept of a point $(x, y, z)$ in a unit cell with dimensions of $a \times b \times c$ is defined by $(x / a, y / b, z / c)$. Miller Indices of (100) in a unit cell with dimensions of $1 \times 1 \times 1$, for example, means that the (100) surface intercepts on $x=$ $1 / 1$ and is parallel to the $y$ - and $z$ - axes. For another example, a (200) surface intercepts on $x=1 / 2$ and is parallel to the $y$ - and $z$ - axes in a unit cell with dimensions of $1 \times 1 \times 1$. Figure II-4 shows the unit cell of the bcc iron and three common iron surfaces: $\mathrm{Fe}(100)$, $\mathrm{Fe}(110)$, and $\mathrm{Fe}(111)$. 
A
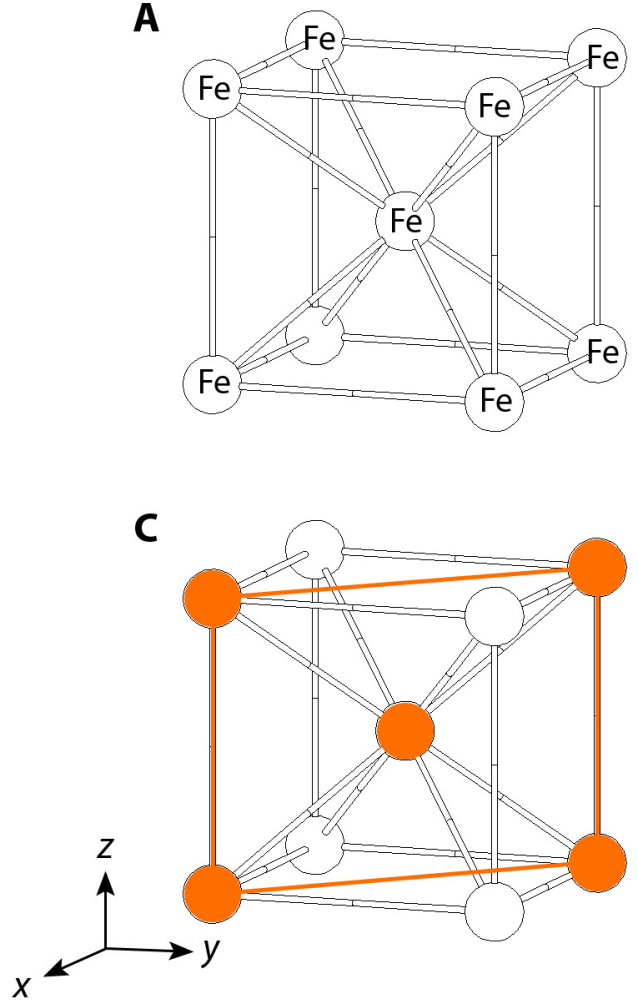

B

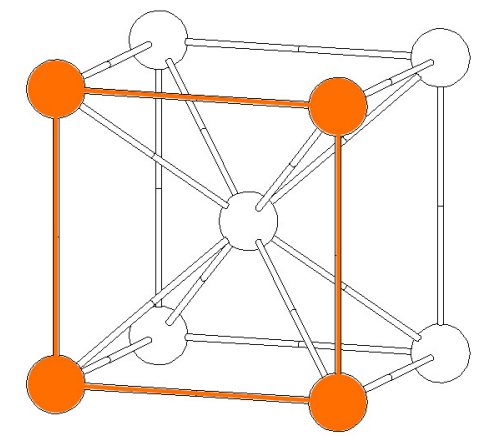

D

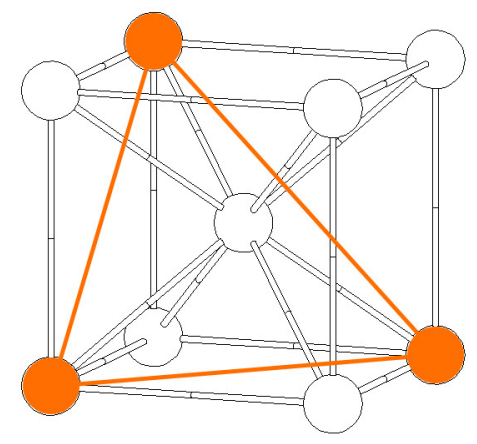

Figure II-4. The unit cell of the body-centered cubic (bcc) iron (A) and $\mathrm{Fe}(100)$ (B), $\mathrm{Fe}(110)(\mathrm{C})$, and $\mathrm{Fe}(111)$ (D) surfaces defined by Miller Indices. Solid lines represent each surface. 


\subsection{Periodic three-layer slab model of $\mathrm{Fe}(110)$ surface}

Among the three common Fe surfaces shown in Figure II-4, Fe(110) is the closestpacked surface of body-centered cubic (bcc) iron. It is essentially bulk-terminated, with very little relaxation and no reconstruction $(21)$. $\mathrm{p}(2 \times 2), \mathrm{p}(3 \times 2)$, and $\mathrm{p}(4 \times 2)$ supercells of $\mathrm{Fe}(110)$ are shown in Figure II-5. The periodic cell lengths of the $\mathrm{p}(3 \times 2)$ and $\mathrm{p}(4 \times 2)$ supercells are 1.5 and 2 times that of the $\mathrm{p}(2 \times 2)$ supercell respectively in the $x$-direction (Figure II-5). The $\mathrm{Fe}(110)$ surface model is periodic three-layer slabs with adsorbates (PCE, TCE or cis-DCE) adsorbed on both sides of the slabs (Figure II-6). This doublesided adsorption model minimizes the dipole moment perpendicular to the surface that is produced by charge rearrangement on the surface due to adsorption. The outermost layers (surface layers) of the slab were allowed to relax, whereas the center layer (below the surface) was kept rigid to represent the underlying bulk iron. The slabs were separated from their periodic images in the $z$-direction by a vacuum space of $16 \AA$ (Figure II-6). Figure II-7 shows the periodic three-layer slabs of Fe(110)-p(3×2). The $p(3 \times 2)$ supercell is replicated four, three, and two times in $x^{-}, y^{-}$, and $z^{-}$directions in Figure II-7.

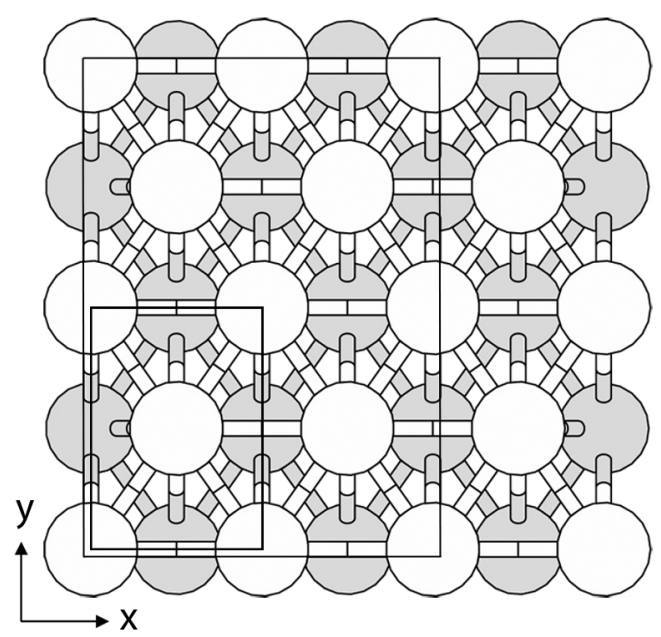

Figure II-5. $p(1 \times 1), p(2 \times 2)$, and $p(3 \times 2)$ supercells of the $\mathrm{Fe}(110)$ surface. The small and big rectangles represent $\mathrm{p}(1 \times 1)$ and $\mathrm{p}(2 \times 2)$, respectively. The entire structure is $\mathrm{Fe}(110)$ $p(3 \times 2)$. The lighter and darker atoms represent the surface and subsurface layers of $\mathrm{Fe}(110)$, respectively. 

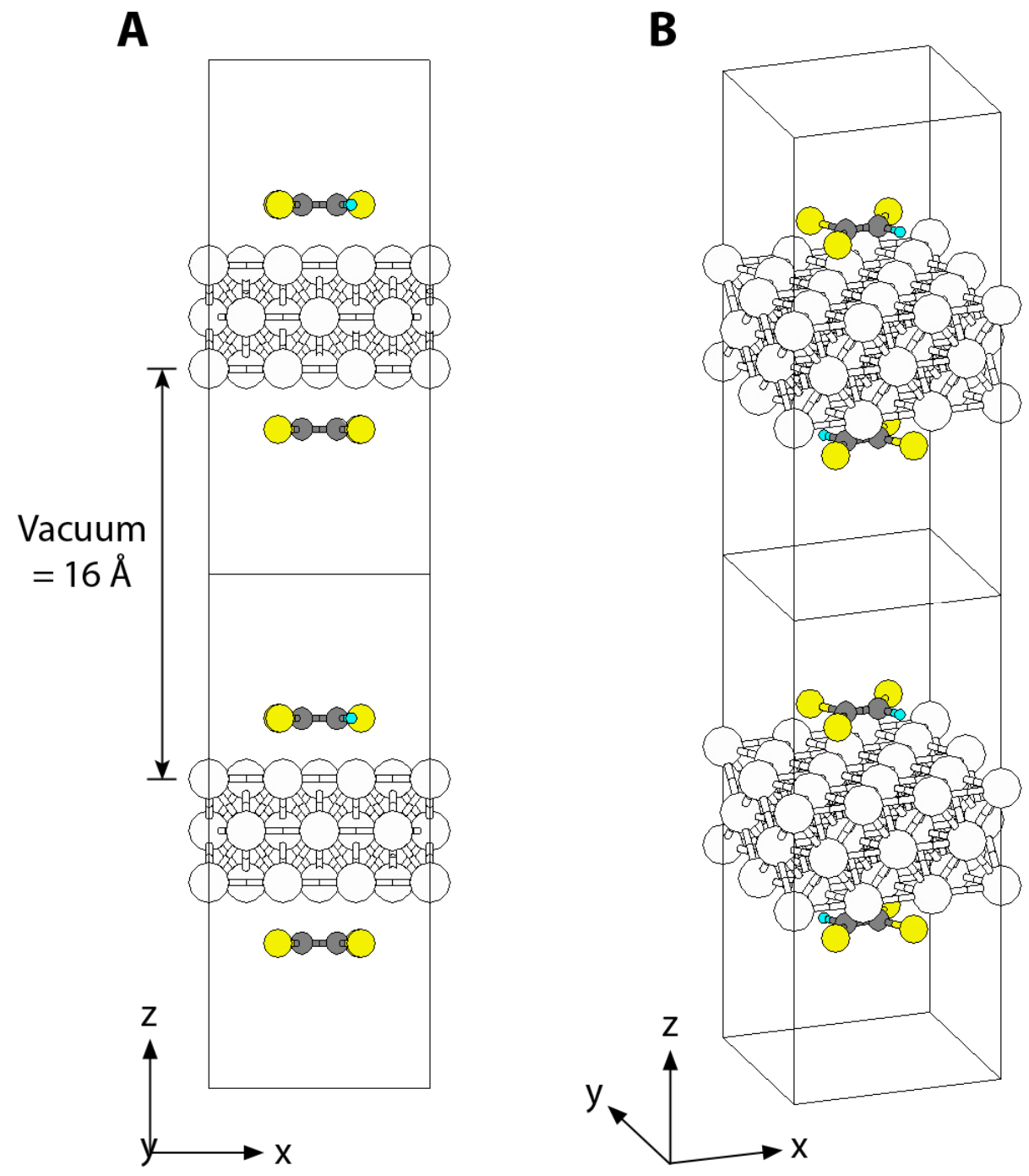

Figure II-6. $\mathrm{Fe}(110)-\mathrm{p}(3 \times 2)$ surface model with vacuum space of $16 \AA$. An intact TCE molecule is located above the surface. (A) Side view of the model. (B) Top side view of the model. 

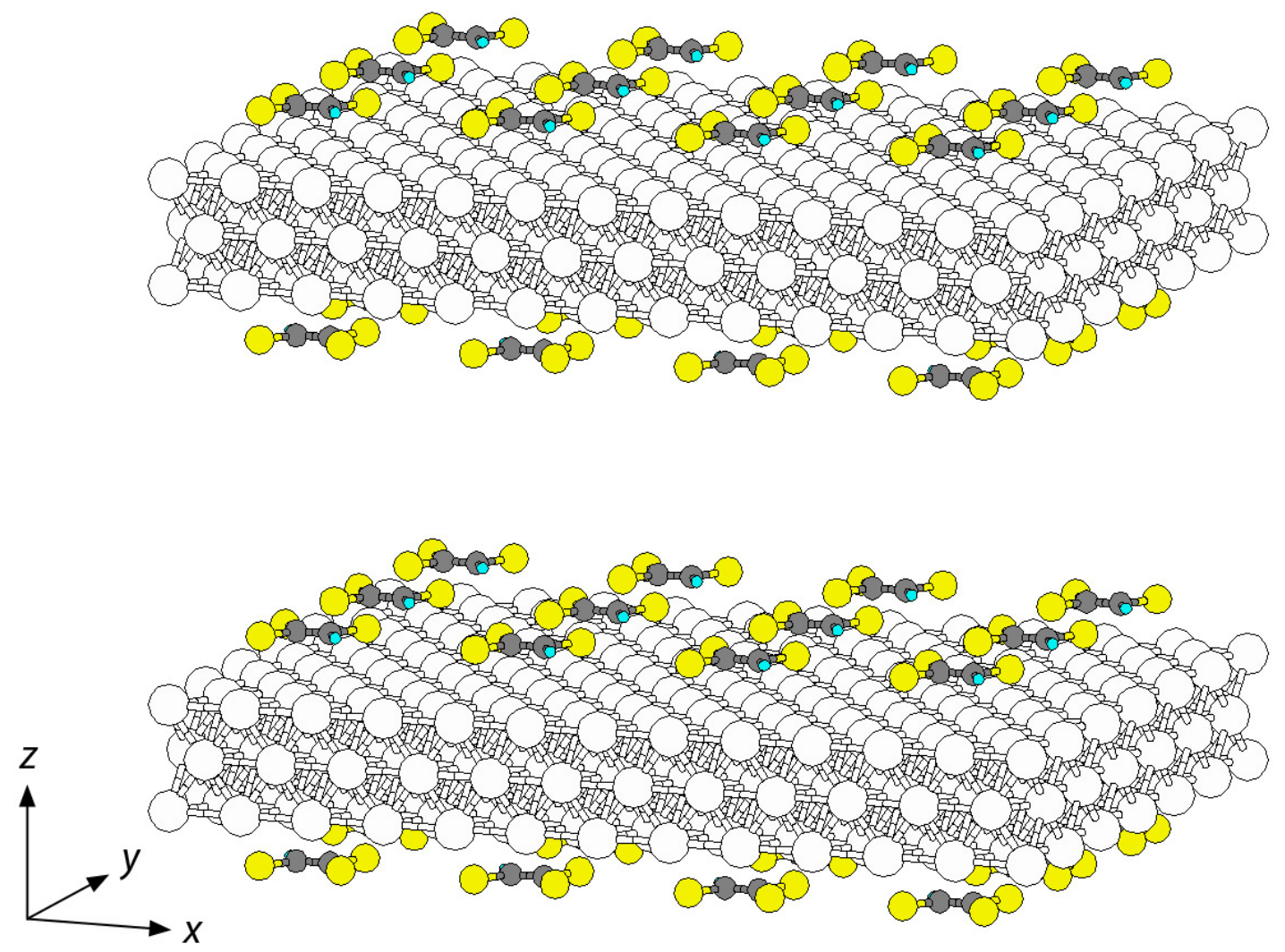

Figure II-7. Periodic three-layer slabs of Fe(110)-p(3×2) with adsorbed TCE. 


\section{References}

(1) Vanderbilt, D., Soft self-consistent pseudopotentials in a generalized eigenvalue formalism. Phys. Rev. B 1990, 41 (11), 7892-7895.

(2) Perdew, J. P.; Burke, K.; Ernzerhof, M., Generalized gradient approximation made simple. Phy. Rev. Lett. 1996, 77 (18), 3865-3868.

(3) Baroni, S.; Dal Corso, A.; de Gironcoli, S.; Giannozzi, P. Quantum ESPRESSO: opEn-Source Package for Research in Electronic Structure, Simulation, and Optimization, v. 3.2; http://www.pwscf.org/: 2007.

(4) Monkhorst, H. J.; Pack, J. D., Special points for brillouin-zone integrations. Phys. Rev. B 1976, 13 (12), 5188-5192.

(5) Ziegler, T., Approximate density functional theory as a practical tool in molecular energetics and dynamics. Chem. Rev. 1991, 91 (5), 651-667.

(6) Kohn, W.; Sham, L. J., Self-consistent equations including exchange and correlation effects. Phys. Rev. 1965, 140 (4A), 1133-\&.

(7) Hohenberg, P.; Kohn, W., Inhomogeneous electron gas. Phys. Rev. 1964, 136 (3B), B864-B871.

(8) Perdew, J. P.; Chevary, J. A.; Vosko, S. H.; Jackson, K. A.; Pederson, M. R.; Singh, D. J.; Fiolhais, C., Atoms, molecules, solids, and surfaces: Applications of the generalized gradient approximation for exchange and correlation. Phys. Rev. B. 1992, 46 (11), 6671-6687.

(9) Hammer, B.; Jacobsen, K. W.; Norskov, J. K., Role of nonlocal exchangecorrelation in activated adsorption. Phy. Rev. Lett. 1993, 70 (25), 3971-3974.

(10) Wu, Z. G.; Cohen, R. E.; Singh, D. J., Comparing the weighted density approximation with the LDA and GGA for ground-state properties of ferroelectric perovskites. Phys. Rev. B 2004, 70 (10).

(11) Filippi, C.; Singh, D. J.; Umrigar, C. J., All-electron local-density and generalizedgradient calculations of the structural-properties of semiconductors. Phys. Rev. B 1994, 50 (20), 14947-14951.

(12) Leach, A. R., Molecular Modelling. Principles and Application. 2nd ed.; Prentice Hall: 2001; p 155.

(13) Arshadi, M. R.; Lashgari, M.; Parsafar, G. A., Cluster approach to corrosion inhibition problems: interaction studies. Mater. Chem. Phys. 2004, 86 (2-3), 311 314.

(14) Frisch, M. J., et al. Gaussian 03, Revision C.02; Gaussian, Inc.: Wallingford CT, 2004.

(15) Wang, G. C.; Ling, J.; Morikawa, Y.; Nakamura, J.; Cai, Z. S.; Pan, Y. M.; Zhao, X. $Z$., Cluster and periodic DFT calculations of adsorption and activation of $\mathrm{CO}_{2}$ on the $\mathrm{Cu}(h k l)$ surfaces. Surf. Sci. 2004, 570 (3), 205-217.

(16) Satoh, S.; Fujimoto, H.; Kobayashi, H., Theoretical study of NH3 adsorption on $\mathrm{Fe}(110)$ and $\mathrm{Fe}(111)$ surfaces. J. Phys. Chem. B 2006, 110 (10), 4846-4852.

(17) Zhou, H.; Tamura, H.; Takami, S.; Kubo, M.; Zhanpeisov, N.; Miyamoto, A., Adsorption properties of $\mathrm{CH}_{3} \mathrm{OH}$ on $\mathrm{Al}(111)$ and $\mathrm{Fe}$ (100) surfaces: A periodic first-principles investigation. Jpn. J. Appl. Phys. 1 2000, 39 (7B), 4275-4278.

(18) Frenkel, D.; Smit, B., Understanding Molecular Simulation. Academic Press: San Diego, 1996. 
(19) Birch, F., Finite elastic strain of cubic crystals. Phys. Rev. 1947, 71 (11), 809-824.

(20) Jiang, D. E.; Carter, E. A., Adsorption and diffusion energetics of hydrogen atoms on Fe(110) from first principles. Surf. Sci. 2003, 547 (1-2), 85-98.

(21) Jiang, D. E.; Carter, E. A., Adsorption and dissociation of CO on Fe(110) from first principles. Surf. Sci. 2004, 570 (3), 167-177. 


\section{CHAPTER III}

\section{ADSORPTION OF CHLOROETHENES ON FE(110)}

\section{Introduction}

Chlorinated solvents such as perchloroethene $\left(\mathrm{PCE}, \mathrm{C}_{2} \mathrm{Cl}_{4}\right)$, trichloroethene (TCE, $\mathrm{C}_{2} \mathrm{HCl}_{3}$ ), and cis-dichloroethene (cis-DCE, $\mathrm{C}_{2} \mathrm{H}_{2} \mathrm{Cl}_{2}$ ) are common groundwater contaminants. PCE and TCE are among the most frequently detected contaminants at hazardous waste disposal sites, due to their widespread production, use and disposal (1, 2), and cause irritation of the skin, eyes, kidneys, and respiratory tract. Moreover, TCE is suspected to be a human carcinogen (3). Chloroethenes released into the subsurface penetrate into the water table and persist as long-term groundwater pollutants $(4,5)$.

Recently, zerovalent iron $\left(\mathrm{Fe}^{0}\right)$ has gained widespread acceptance as an effective reducing agent for groundwater remediation at sites contaminated with chloroethenes (68 ), on account of its low cost and nontoxicity (9). The mechanisms by which iron decomposes chlorinated solvents, by catalytic cleavage of the carbon-chlorine bond (9), are as yet not well understood. To develop process models for the optimal design of in situ and ex situ zerovalent iron treatment systems for the removal of chlorinated solvents from drinking water supplies, it is important to understand these mechanisms, and in particular how the degree of chlorination of the contaminant affects its reactivity on the iron surface (10). Generally, it is assumed that reactivity should increase with increasing chlorination (6). For example, the dechlorination rate of PCE was reported to be 5.4 times faster on zerovalent iron $(11,12)$ and 170.2 times faster on zerovalent zinc $(10)$ than that of TCE. A higher degree of chlorination was also found to favor rapid dechlorination on iron-bearing soil minerals such as biotite (13), magnetite (14), and green rust (15).

However, the opposite trend has also been reported for decomposition of chloroethenes by iron (6), with a measured overall order of reactivity for chloroethene reduction by zerovalent iron given as vinyl chloride $(\mathrm{VC})>$ cis-DCE $>$ TCE $>$ PCE (の). A similar ordering was obtained for dechlorination of chloroethenes by palladium metal 
catalysts $(\mathrm{Pd} / \mathrm{Al})$ with hydrogen gas (16). In another study, TCE was transformed 2.6 times faster than PCE by iron sulfide (17). Hence, contradictory results have been reported for the reactivity ordering with respect to degree of chlorination for chloroethene reduction on metals and metal-containing minerals.

Various explanations have been offered for the conflicting results for chloroethene reactivity on zerovalent iron. One possibility is that carbon impurities within the iron may serve as nonreactive sites on the iron surface where chloroethenes may adsorb without further reaction. The adsorbed chloroethene may thus be misinterpreted as a part of the overall reaction rate (18). Highly chlorinated species such as PCE have a greater propensity to adsorb on carbon than less-chlorinated congeners, and this adsorption may easily be mistaken for rapid reaction $(6,18)$. However, because of the difficulty in performing quantitatively accurate experiments, it remains unresolved how the degree of chlorination affects the relative reactivity of chloroethenes on iron surfaces.

In the studies reported herein, density functional theory (DFT) methods were applied to investigate the adsorption configurations of chloroethenes on the $\mathrm{Fe}$ (110) surface. This was done as a first step toward elucidating the effect of chlorination on the relative reactivity of chloroethenes on zerovalent iron. Reductive dechlorination of chloroethenes may occur by a three-step process, involving adsorption of the chloroethene onto the iron surface; a surface-catalyzed reduction; and desorption of the dechlorinated product species from the surface. To determine the relative reactivity of chloroethene congeners on zerovalent iron, differentiation between the adsorption and dechlorination steps is needed so as to prevent misinterpretation of chloroethene adsorption as a part of the overall dechlorination rate. Since chemical reactions between chloroethenes and the iron surface are initiated after the molecules have adsorbed, accurate characterization of the adsorption sites and energies is an important initial step toward analyzing the surface dechlorination pathways of chloroethenes bound to zerovalent iron (19). In this chapter, we therefore present and discuss theoretical results from DFT calculations for the adsorption energies and electronic properties of chloroethenes adsorbed on the Fe(110) surface. 


\section{Computational Methods}

\subsection{Adsorption Calculations using Periodic Boundary Conditions.}

All electronic structure calculations were performed using periodic density functional theory and the ultrasoft Vanderbilt pseudopotential method (20) within the generalized gradient approximation (GGA) by Perdew, Burke, and Ernzerhof (PBE) (21) for the exchange-correlation functional as implemented in the Plane-Wave SelfConsistent Field (PWscf) code in the Quantum-ESPRESSO open-source distribution (22). Kinetic-energy cutoffs for wavefunctions and for charge density and potential were $340.1 \mathrm{eV}$ (25 Ryd) and $2449.0 \mathrm{eV}$ (180 Ryd), respectively; this converges the total energy to within $0.01 \mathrm{eV} /$ atom. The Monkhorst-Pack scheme (23) was used for the $k$-point sampling. Using a $10 \times 10 \times 10 k$-point mesh, the following physical properties were calculated for ferromagnetic bcc iron: equilibrium lattice constant $a_{0}=2.8606 \AA$; bulk modulus $B=167 \mathrm{GPa}$; and magnetic moment $M=2.45 \mu_{\mathrm{B}}$. These results are in good agreement with experimental results (24) for ferromagnetic bcc iron: $a_{0}=2.8664 \AA, B=$ $168 \mathrm{GPa}, M=2.22 \mu_{\mathrm{B}}$.

$\mathrm{Fe}(110)$ is the closest-packed surface of body-centered cubic (bcc) iron. It is essentially bulk-terminated, with very little relaxation and no reconstruction $(25) \cdot \mathrm{p}(2 \times 2)$, $\mathrm{p}(3 \times 2)$, and $\mathrm{p}(4 \times 2)$ supercells of $\mathrm{Fe}(110)$ were modeled using periodic three-layer slabs with chloroethenes (PCE, TCE or cis-DCE) adsorbed on both sides of the slabs. This double-sided adsorption model minimizes the dipole moment perpendicular to the surface that is produced by charge rearrangement on the surface due to adsorption. The outermost layers (surface layers) of the slab were allowed to relax, whereas the center layer (below the surface) was kept rigid to represent the underlying bulk iron. The coverages of each adsorbate molecule were $0.125,0.083$, and 0.063 monolayers (ML) respectively for the $\mathrm{p}(2 \times 2), \mathrm{p}(3 \times 2)$, and $\mathrm{p}(4 \times 2)$ supercells. The periodic cell lengths of the $\mathrm{p}(3 \times 2)$ and $\mathrm{p}(4 \times 2)$ supercells are 1.5 and 2 times that of the $\mathrm{p}(2 \times 2)$ supercell respectively in the $x$-direction (Figure III-1). The slabs were separated from their periodic images in the $z$-direction by a vacuum space of $16 \AA$. Brillouin-zone integrations were performed on a $2 \times 3 \times 1$ Monkhorst-Pack grid of $k$-points for the $\mathrm{Fe}(110)$ supercells. The $2 \times 3 \times 1 k$-point grid 
yielded adsorption energies close (less than $2.2 \mathrm{~kJ} / \mathrm{mol}$ underestimation) to those obtained using the more computationally expensive $5 \times 5 \times 1 \mathrm{k}$-point grid.

Chloroethene adsorption was considered at four different candidate adsorption sites and orientations. These are the atop 1, atop 2, long-bridge (LB), and short-bridge (SB) sites shown in Figure III-1. The adsorption energy $\left(E_{a d s}\right)$ per chloroethene molecule adsorbed to the iron surface is calculated as

$$
E_{\text {ads }}=\frac{1}{N_{\text {adsorbate }}}\left[E_{\text {adsorbate/surface }}-\left(E_{\text {adsorbate }}+E_{\text {surface }}\right)\right]
$$

where $N_{\text {adsorbate }}$ is the number of chloroethene molecules in the model system, and $E_{\text {adsorbate/surface, }} E_{\text {adsorbate, }}$, and $E_{\text {surface }}$ represent the total energies of the chloroethene/iron system, the free chloroethene molecule, and the clean iron surface, respectively. The geometry of the free adsorbate molecule was optimized in a $12 \AA$ cubic box, where little interaction is expected between the gas phase chloroethene and its periodic images. A negative adsorption energy indicates that adsorption is exothermic (stable) with respect to the free adsorbate molecule.

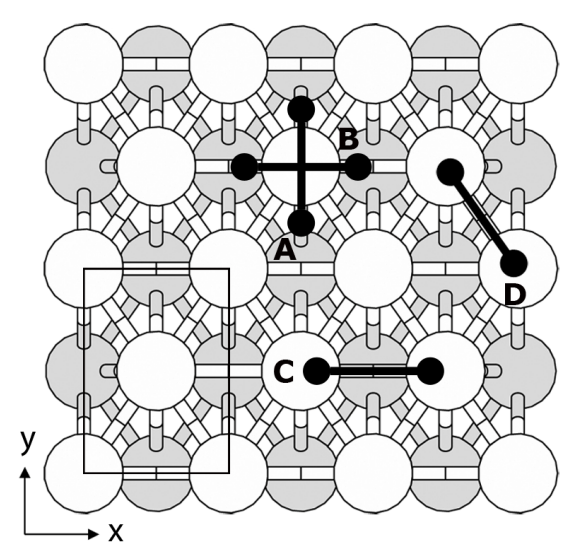

Figure III-1. $\mathrm{Fe}(110)-\mathrm{p}(3 \times 2)$ supercell and adsorption sites used for chloroethene adsorption calculations. The rectangle demarcates $\mathrm{p}(1 \times 1)$ and the solid line denotes the orientation of the chloroethene $\mathrm{C}=\mathrm{C}$ bond. The lighter and darker atoms represent the surface and subsurface layers of $\mathrm{Fe}(110)$, respectively. (A) atop 1 site. (B) atop 2 site. (C) long-bridge (LB) site. (D) short-bridge (SB) site. 
Geometry optimizations are halted when the forces acting on each relaxed atom of the system were smaller than a relatively lax convergence criterion of $5.14 \times 10^{-1} \mathrm{eV} / \AA$ $\left(2 \times 10^{-2} \mathrm{Ryd} / \mathrm{a}\right.$.u. $)$. Owing to the relatively lax convergence criterion, geometry optimization of adsorbed chloroethenes on the Fe surface was terminated keeping an angle of less than $10^{\circ}$ between the $\mathrm{C}=\mathrm{C}$ bond axis and the Fe surface. This is based on experimental results indicating that the $\mathrm{C}=\mathrm{C}$ bond axis of adsorbed chloroethenes on $\mathrm{Pt}$ (1) and vinyl bromide on $\mathrm{Cu}$ (2) lie parallel to the metal surfaces. A force convergence criterion that is too stringent may result in detachment of chlorine atoms from the chloroethene backbone, distorting the parallel alignment of the $\mathrm{C}=\mathrm{C}$ bond with the surface. Figure III-2 shows the maximum force and the relative total energy of TCE for each optimization step for TCE adsorption on the SB site of Fe(110). At the start of the geometry optimization, the TCE molecule is planar. Using the lax convergence criterion, the total energy and maximum force achieve convergence in the $11^{\text {th }}$ step of the geometry optimization. If a more stringent convergence criterion is used, so that the optimization is continued beyond the $11^{\text {th }}$ step, the total energy continuously decreases and the maximum force significantly increases after the $19^{\text {th }}$ step of the optimization. This step corresponds to the onset of chlorine atom detachment from the $\mathrm{C}=\mathrm{C}$ backbone of TCE and stabilizing attachment to the Fe surface. Thus, the adsorbed state of the intact TCE molecule is realized in the $11^{\text {th }}$ to $19^{\text {th }}$ steps of the geometry optimization step shown in Figure III-2, and hence, the chloroethene adsorption energy on the $\mathrm{Fe}(110)$ surface is determined for this and other geometry optimizations using the geometry of the adsorbed species at the first step for which the lax convergence criterion is satisfied. The optimized structures were visualized using the XCrySDen graphical package (26). 


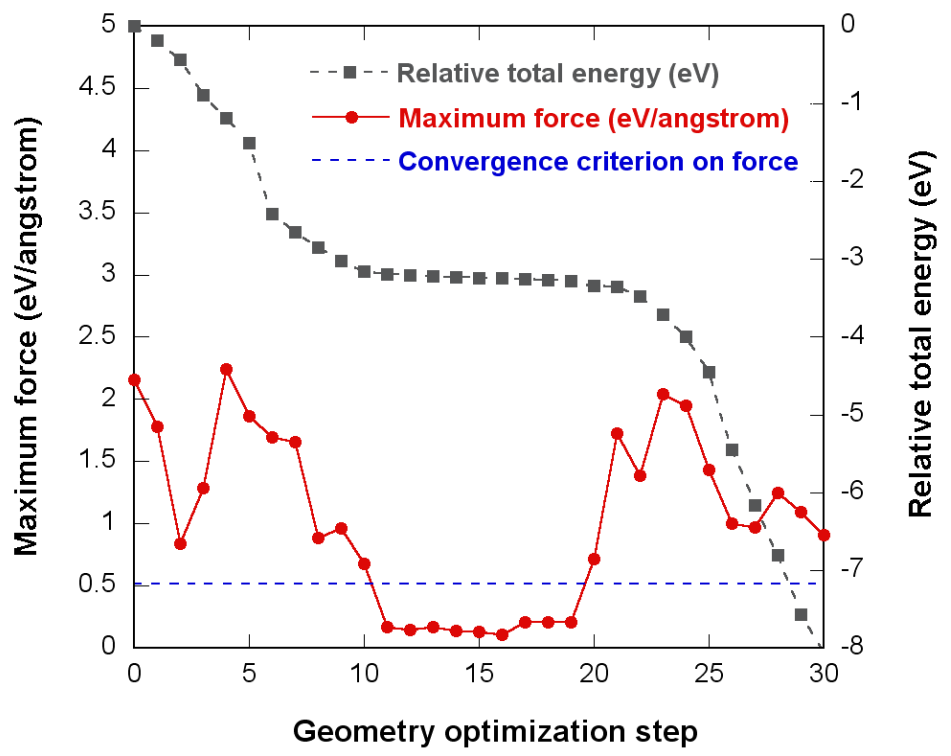

Figure III-2. Maximum force and relative total energy calculated for TCE adsorption at the short-bridge (SB) site of Fe(110) during geometry optimization. 


\subsection{Cluster Model Analysis.}

After obtaining the adsorbed chloroethene configurations from periodic slab calculations using Quantum-ESPRESSO, the coordinates of the adsorbed chloroethenes and neighboring iron surface atoms were transferred to Gaussian 03 (27) to calculate single point energies and to identify important bonding orbitals between the $\mathrm{C}=\mathrm{C}$ bond and the Fe surface. The GGA of PBE for the exchange-correlation functional was used

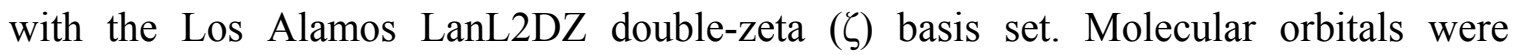
visualized using the MOLDEN molecular density viewer (28). Hydration energies of the chloroethenes were calculated using the polarized continuum model (PCM) (29) implemented in Gaussian 03 with the same functional method and basis set as used in the bonding orbital analyses. 


\section{Results and Discussion}

\subsection{Effect of supercell size and k-point grid on adsorption}

To design a proper iron surface model, the effect of supercell size was studied for the $\mathrm{p}(2 \times 2), \mathrm{p}(3 \times 2)$, and $\mathrm{p}(4 \times 2)$ supercells of $\mathrm{Fe}(110)$ with adsorbates at the atop 1 site. This site was selected for analysis because it exhibited the most energetically favorable adsorption configurations for chloroethenes in a preliminary study using the $\mathrm{Fe}(110)$ $\mathrm{p}(2 \times 2)$ supercell with a fixed iron surface layer (data not shown here). The $\mathrm{p}(3 \times 2)$ supercell was found to be a reasonable supercell size for obtaining property results comparable to larger supercells while reducing the computational burden associated with the DFT calculations (Figure III-3). The adsorption energies of the chloroethenes on the $\mathrm{Fe}(110)$ atop 1 site decrease (i.e., become more exothermic) with increasing supercell size, as repulsive interactions between the adsorbate and its periodic images are reduced. A similar trend was observed for oxygen adsorption on $\mathrm{Cu}(111)$ as coverage was varied (30). In addition, adorption at the LB and SB sites of the Fe(110)-p(2×2) supercell with a fixed surface yielded endothermic (unstable) chloroethene adsorption energies (data not shown here). Although the adsorption energy on the fixed $\mathrm{p}(2 \times 2)$ supercell surface is less than $9 \mathrm{~kJ} / \mathrm{mol}$ larger than that for the relaxed $\mathrm{p}(2 \times 2)$ surface (Figure III-3), the size of the $\mathrm{p}(2 \times 2)$ supercell is insufficient to determine chloroethene adsorption energies because of repulsive interactions between the periodic images of these relatively large adsorbates on the iron surface.

For larger supercells, the calculated adsorption energies are progressively more negative, with the largest differences in the adsorption energies obtained for the $\mathrm{p}(2 \times 2)$ and $\mathrm{p}(3 \times 2)$ supercells occurring for PCE and the smallest for cis-DCE. The latter exhibits almost no difference $(0.04 \mathrm{~kJ} / \mathrm{mol})$, whereas the former exhibits a dramatic change $(36.1 \mathrm{~kJ} / \mathrm{mol})$ and TCE shows a moderate change $(18.0 \mathrm{~kJ} / \mathrm{mol})$. For the more highly chlorinated chloroethenes, periodicity in a small supercell results in significant repulsion between the chlorines of image pairs due to the short $\mathrm{Cl}-\mathrm{Cl}$ distances. A slight additional reduction in the periodicity effect is achieved by using a larger $\mathrm{p}(4 \times 2)$ supercell, but at substantial computational expense since the calculations scale approximately with the cube of the number of electrons. Adsorption energy calculations 
for the $\mathrm{p}(4 \times 2)$ supercell require triple the resources used for the $\mathrm{p}(3 \times 2)$ supercell. Since the purpose of this study is to investigate the effect of chlorination number on chloroethene adsorption on the $\mathrm{Fe}(110)$ surface, and since the differences in the PCE, TCE and cis-DCE adsorption energies are similar for the $\mathrm{p}(3 \times 2)$ and $\mathrm{p}(4 \times 2)$ supercells (Figure III-3), the adsorption results and analyses that follow were obtained using the $\mathrm{p}(3 \times 2)$ supercell, which gives a satisfactory reduction of periodicity effects at a reasonable computational effort.

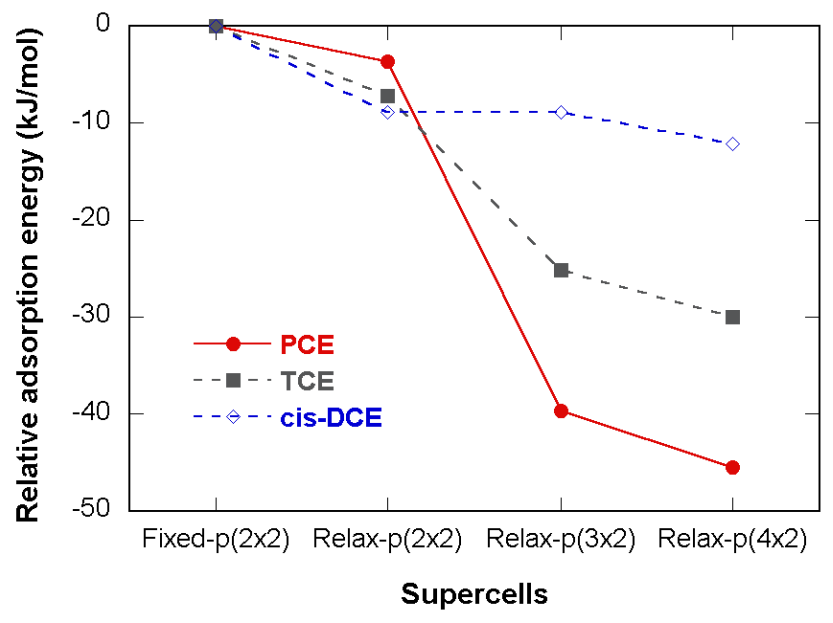

Figure III-3. Relative adsorption energies of PCE, TCE, and cis-DCE from the gas phase onto the atop 1 site of $\mathrm{Fe}(110)$, for different surface relaxation conditions and different supercell sizes. (A tight convergence criterion $\left(2.57 \times 10^{-1} \mathrm{eV} / \AA\left(10^{-2} \mathrm{Ryd} / \mathrm{a} . u\right.\right.$. $\left.)\right)$ on the forces acting on each relaxed atom of the system and a $5 \times 5 \times 1 \mathrm{k}$-point grid were used in the adsorption energy calculations. For geometry optimizations at the atop 1 site, the chloroethene $\mathrm{C}=\mathrm{C}$ bond axis maintains an orientation parallel to the Fe surface, even when the tight convergence criterion on the forces is applied).

The effect of the $k$-point grid size was also sampled to determine the smallest $k$ point grid that produces reliable adsorption results. Since the infinite periodic system is described using only a finite number of available energy levels occupied at each $k$-point, one should determine the number of $k$-points that yields convergence of the properties of interest. As with supercell size, larger numbers of $k$-points generally produce more accurate adsorption energies but require more computational effort. To determine a suitable $k$-point grid for the adsorption studies, the adsorption energies of chloroethenes at atop 1 site of the $\mathrm{Fe}(110)-\mathrm{p}(3 \times 2)$ supercell were calculated for a selection of different 
$k$-point grids. Sorption energies for PCE, TCE and cis-DCE are shown relative to the energies obtained for the $5 \times 5 \times 1 k$-point grid in Figure III-4. A coarse $k$-point grid, $3 \times 3 \times 1$, slightly underestimates the PCE and TCE adsorption energies and overestimates the $c i s$-DCE adsorption energy (less than $0.7 \mathrm{~kJ} / \mathrm{mol}$ ) compared with the $5 \times 5 \times 1$ grid. An even coarser grid, $2 \times 3 \times 1$, yields sorption energies close to those of the $5 \times 5 \times 1 \mathrm{k}$-point grid, with less than $2.2 \mathrm{~kJ} / \mathrm{mol}$ difference from the baseline energies from all three chloroethenes. Moreover, the ordering of the adsorption energies of PCE, TCE, and cisDCE is unchanged when the smaller $3 \times 3 \times 1$ and $2 \times 3 \times 1 k$-point grids are used instead of the larger and more computationally expensive $5 \times 5 \times 1$ and $7 \times 7 \times 1$ grids. Therefore, in this study, the $2 \times 3 \times 1 \mathrm{k}$-grid point was used to calculate the chloroethene adsorption energies and activation energies.

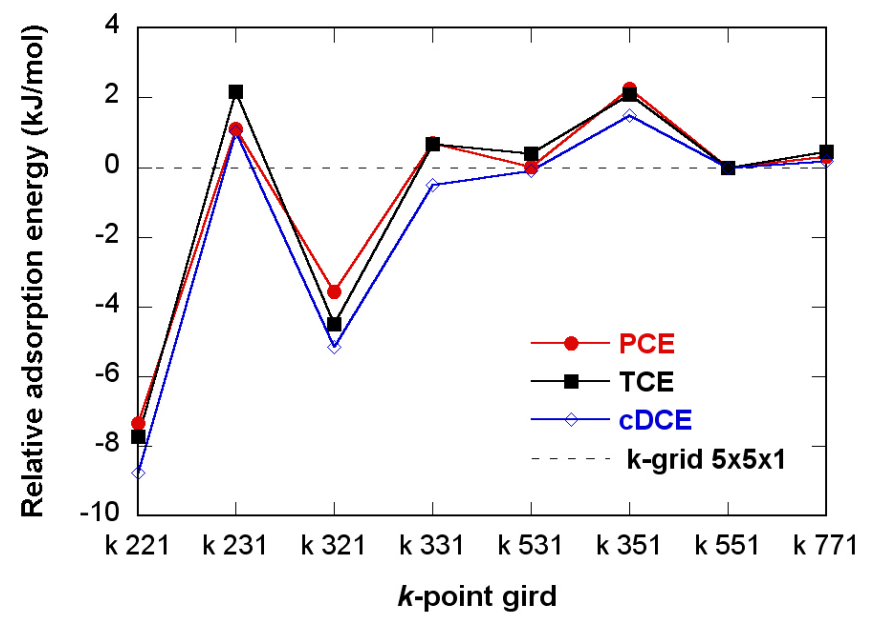

Figure III-4. Relative adsorption energies of PCE, TCE, and cis-DCE from the gas phase on the atop 1 site of the $\mathrm{Fe}(110)$-p $(3 \times 2)$ supercell for different $k$-grids. 


\subsection{Chloroethene adsorption on $\mathrm{Fe}(110)-\mathrm{p}(3 \times 2)$}

Surface relaxation of the three-layered clean Fe(110) slab was carried out and the final geometry was compared with experimental data to verify the iron surface model. Lateral relaxation of $\mathrm{Fe}$ atoms in the $x$ - and $y$-direction was $0.008 \%$ and $0.007 \%$, respectively, relative to the distance between Fe atoms in the fixed surface model. This was negligible compared with relaxation normal to the surface (i.e., in the $z$-direction) and indicates that the $\mathrm{Fe}(110)$ surface does not reconstruct, in agreement with experimental and theoretical results $(24,31,32)$. Surface relaxation is determined by measuring the distance the first layer moves $(\delta z)$. A positive and a negative value represent expansion and contraction of the surface layer, respectively. The average relaxation $\delta z$ of the surface $\mathrm{Fe}(110)$ atoms as a percentage of the interlayer spacing was 0.15 , where the negative value indicates contraction of the surface layer toward the subsurface layer $\left(3 \times 10^{-3} \AA\right.$ contraction). This is in agreement with a quantum mechanical study $(\delta z=-0.13)(32)$ and falls within the range reported from experimental measurement $(\delta z=+0.5 \pm 2)(31)$.

Chloroethenes with gas-phase optimized geometries were placed on the four adsorption sites (atop 1, atop 2, LB and SB) shown in Figure III-1 of relaxed-surface $\mathrm{Fe}(110)-\mathrm{p}(3 \times 2)$ and re-optimized to calculate the adsorption energies. As indicated in Table III-1, the atop 1 site exhibits the most energetically favorable adsorption configuration for all three chloroethenes investigated, with adsorption energies on this site that are 9 to $58 \mathrm{~kJ} / \mathrm{mol}$ more negative than those of other adsorption sites. The adsorbed configurations of PCE, TCE, and cis-DCE, shown in Figure III-5, deviate significantly from their planar gas phase geometries. The $\mathrm{C}=\mathrm{C}$ bond approaches the $\mathrm{Fe}$ surface to within 1.85 to $1.91 \AA$, whereas the $\mathrm{C}-\mathrm{Cl}$ and $\mathrm{C}-\mathrm{H}$ bonds deflect away from the surface. The latter is reflected in a reduction of the $180.0^{\circ}$ gas-phase chloroethene $\mathrm{Cl}-\mathrm{C}-\mathrm{C}-\mathrm{Cl}$ dihedral angle to $122.0^{\circ}$ to $131.9^{\circ}$ for chloroethenes adsorbed on the $\mathrm{Fe}(110)$ surface (Table III-2). Also, the $\mathrm{C}=\mathrm{C}$ bond and $\mathrm{C}-\mathrm{Cl}$ bonds of adsorbed chloroethenes are elongated by 0.11 to $0.14 \AA$ relative to their gas-phase geometries (Table III-2).

The adsorbed configurations thus reveal $s p^{2}-s p^{3}$ hybridization of the chloroethene carbon atoms, in agreement with the adsorption geometry found for TCE on $\mathrm{PdCu}(110)$ (33). The geometry changes of the adsorbed chloroethenes demonstrate strong activation 
for dechlorination on the $\mathrm{Fe}(110)$ surface. Similar structural changes were reported in the adsorption of ethylene $\left(\mathrm{C}_{2} \mathrm{H}_{4}\right)$ on various metal surfaces (34-39). Bernardo and Gomes (34) suggest that the adsorption of ethylene occurs mainly on the atop site of $\mathrm{Cu}(110)$, $\operatorname{Ag}(110)$, and $\operatorname{Pt}(110)$ surfaces, indicating carbon-carbon bond elongation. This bond elongation, accompanied by partial hybridization toward the $s p^{3}$ configuration, is a common phenomenon in the adsorption of ethylene on $\operatorname{Ag}(100)$ (35), $\operatorname{Pd}(110)$ (36), $\mathrm{Pt}(111)$ (37), $\mathrm{Ag}(111$ ) (38), and $\mathrm{Pd}(111)$ (39). Zhang et al. (40), however, reported a $\mathrm{C}=\mathrm{C}$ bond contraction by $0.043 \AA$ upon adsorption of TCE on the most energetically favorable site of $\mathrm{Fe}(100)$. This is probably because the $\mathrm{C}-\mathrm{Cl}$ bonds are highly elongated (3.418 $\AA$ ), such that the adsorbed TCE molecule acts as if it is in a dissociated configuration, with very little overlap in electron density between the $\mathrm{C}$ and $\mathrm{Cl}$ atoms and a $\mathrm{C}=\mathrm{C}$ bond that is closer in character to a $\mathrm{C} \equiv \mathrm{C}$ triple bond.

The adsorbed configurations of the chloroethenes may directly affect the relative activation energies among the different possible dechlorination pathways. The $\mathrm{C}-\mathrm{Cl}$ bond length $(1.850 \AA)$ on the $\mathrm{CCl}_{2}$ group of TCE is, for example, more elongated, and hence more activated for dechlorination, than that of the $\mathrm{CHCl}$ group $(\mathrm{C}-\mathrm{Cl}$ bond elongation of $1.822 \AA$ ). Barbosa and Sautet (41) similarly report that $\mathrm{C}-\mathrm{Cl}$ bond dissociation of TCE is more favorable from the $\mathrm{CCl}_{2}$ group than the $\mathrm{CHCl}$ group. 
Table III-1. Adsorption energies of chloroethenes from the gas phase and the aqueous phase $^{a}$ on $\mathrm{Fe}(110)-\mathrm{p}(3 \times 2)$ surface with a $2 \times 3 \times 1 k$-point grid

\begin{tabular}{cccccc} 
& \multirow{2}{*}{$\begin{array}{c}\text { hydration } \\
\text { energy } \\
\text { (kJ/mol) }\end{array}$} & \multicolumn{4}{c}{ adsorption energy (kJ/mol) } \\
\cline { 3 - 6 } & & atop 1 & atop 2 & LB & SB \\
PCE & -4.5 & -69.7 & -60.9 & -12.0 & -44.7 \\
& & $(-67.5)$ & $(-58.7)$ & $(-9.7)$ & $(-42.5)$ \\
TCE & -13.1 & -69.2 & -56.4 & -17.8 & -49.7 \\
& & $(-62.6)$ & $(-49.8)$ & $(-11.3)$ & $(-43.1)$ \\
cis-DCE & -24.0 & -66.5 & -43.1 & -21.5 & -39.4 \\
& & $(-54.5)$ & $(-31.1)$ & $(-9.5)$ & $(-27.4)$ \\
\hline
\end{tabular}

${ }^{a}$ Values in parentheses are adsorption energies for aqueous-phase chloroethenes, calculated assuming a 50\% partial hydration for chloroethenes adsorbed on the iron surface. 

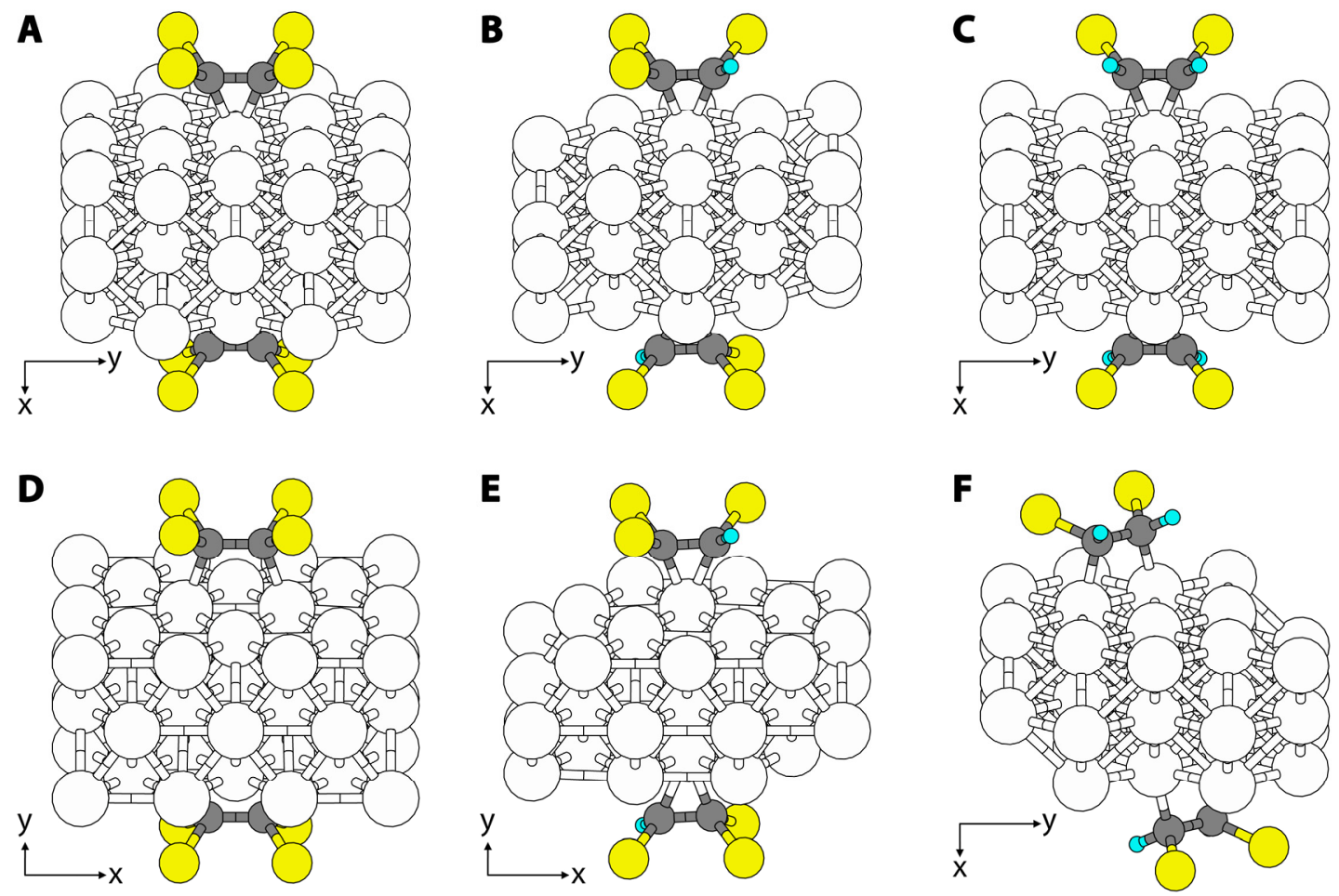

$\bigcirc \mathrm{Fe} \bigcirc \mathrm{Cl} \bigcirc \mathrm{C} \circ \mathrm{H}$

Figure III-5. Adsorbed configurations of PCE, TCE, and cis-DCE on the relaxed $\mathrm{Fe}(110)-\mathrm{p}(3 \times 2)$ surface. (A) PCE at atop 1. (B) TCE at atop 1. (C) cis-DCE at atop 1. (D) PCE at long-bridge (LB). (E) TCE at atop 2. (F) cis-DCE at short-bridge (SB). 
Table III-2. Bond lengths $(\AA)$ and dihedral angles $\left(^{\circ}\right)$ of chloroethenes in the gas phase and adsorbed on the atop 1 site of the relaxed Fe(110)-p(3×2) surface

\begin{tabular}{|c|c|c|c|c|c|c|c|c|}
\hline & \multicolumn{4}{|c|}{ gas phase } & \multicolumn{4}{|c|}{ adsorbed phase } \\
\hline & $\mathrm{C}=\mathrm{C}$ & $\mathrm{C}-\mathrm{Cl}$ & $\mathbf{C}-\mathbf{H}$ & dihedral & $\mathbf{C}=\mathbf{C}$ & $\mathrm{C}-\mathrm{Cl}$ & $\mathbf{C}-\mathbf{H}$ & dihedral \\
\hline PCE & 1.357 & 1.715 & - & 180.0 & 1.480 & 1.857 & - & 122.0 \\
\hline TCE & 1.346 & $\begin{array}{l}1.713^{a} \\
1.715^{b} \\
1.727\end{array}$ & 1.091 & 180.0 & 1.460 & $\begin{array}{l}1.822^{a} \\
1.829^{b} \\
1.850\end{array}$ & 1.103 & $\begin{array}{l}131.9^{c} \\
122.6^{d}\end{array}$ \\
\hline cis-DCE & 1.340 & 1.716 & 1.092 & 180.0 & 1.478 & 1.857 & 1.113 & 124.9 \\
\hline
\end{tabular}

${ }^{a} \mathrm{C}-\mathrm{Cl}$ bond on the carbon bonded to a hydrogen atom

${ }^{b} \mathrm{C}-\mathrm{Cl}$ bond on the carbon bonded to two chlorine atoms

${ }^{c} \mathrm{Cl}-\mathrm{C}-\mathrm{C}-\mathrm{Cl}$ dihedral angle

${ }^{d} \mathrm{H}-\mathrm{C}-\mathrm{C}-\mathrm{Cl}$ dihedral angle 


\subsection{Hydration Energy}

For investigation of adsorption and dissociation of chloroethenes on zerovalent iron in groundwater remediation applications, it is important, albeit challenging, to take into account solvent effects in quantum simulations of metal surface-catalyzed reactions. The thermodynamics and kinetics of surface reactions can be significantly modified in the presence of a solvent because partially charged intermediates that are unstable in the gas phase can exist in solution and participate in the reactions $(40,42)$. However, explicit inclusion of solvent effects in quantum simulations requires huge computational resources, especially when relatively large adsorbate molecules are investigated on transition metal surfaces such as in this study. Thus, only the hydration energies of chloroethene molecules in bulk water are used in this study to take solvent effects into account. Explicit calculation of the partial hydration of the adsorbate on the surface is neglected because of the computational expense of calculating the hydration of the entire iron-chloroethene cluster. The adsorption energy in the presence of water can be approximated by calculating adsorption in the gas phase and correcting for the loss in hydration energy of the chloroethenes.

The hydration energies of PCE, TCE, and cis-DCE calculated using the PCM method as implemented in Gaussian 03 (27) are -4.5, -13.1, and -24.0 kJ/mol (Table III1). Since the enthalpy of hydration of the hydrogen ion $(-1106.7 \mathrm{~kJ} / \mathrm{mol})$ is more negative, due to the formation of hydrogen bonds, than that of the chlorine ion $(-340.2$ $\mathrm{kJ} / \mathrm{mol}$ ) (43), hydration energy decreases as the number of hydrogen atoms increases. Negative hydration energies indicate that chloroethenes must overcome the loss of hydration energy upon adsorption, resulting in less exothermic (or more endothermic) adsorption energies from the aqueous phase. To estimate the hydration energies of the adsorbed species, Becker and coworkers (44) used molecular dynamics simulations to determine the adsorption energies of species on sulfate surfaces. They determined that the hydration energy of $\mathrm{a} \mathrm{Ba}^{2+}$ ion on a corner site of the barite $\left(\mathrm{BaSO}_{4}\right)$ surface is about $20 \%$ of the $\mathrm{Ba}^{2+}$ hydration energy in bulk water (44). This is because the ion is less exposed to solvent water in the corner adsorption site. For a chloroethene molecule adsorbed on a planar Fe(110) surface, it is reasonable to a first approximation to assume that the hydration energy of the adsorbed molecule is $50 \%$ of the hydration energy of the 
same species in bulk water. Using the gas phase adsorption energy $\left(E_{\text {ads_gas }}\right)$ and the bulk water hydration energy ( $\left.E_{\text {hyd_bulk }}\right)$ of the chloroethene, adsorption energies from the aqueous phase $\left(E_{\text {ads_water }}\right)$ can be calculated as

$$
E_{\text {ads_water }}=E_{\text {ads_gas }}-\left(p \times E_{\text {hyd_bulk }}\right)
$$

where $p$ is the fractional solvation of the adsorbed molecule (here assumed to be $50 \%$ or $p=0.5)$.

Adsorption energies of chloroethenes from water are reported as the values in parentheses in Table III-1. PCE shows stronger adsorption from water on the $\mathrm{Fe}(110)$ surfaces than TCE and cis-DCE in agreement with experimental results showing that aqueous adsorption constants for chloroethenes increase with increasing halogenation ( 6 , 13-15, 18). Arnold and Roberts (6) report similar results, although the adsorption constant they obtained for PCE is smaller than that for TCE. Lee and Batchelor (13-15) also showed that the adsorption constants of chloroethenes on reactive iron-bearing soil minerals increase with increasing chlorination.

Although the magnitude of difference in adsorption energy is small $(\sim 3 \mathrm{~kJ} / \mathrm{mol})$ among the chloroethenes in the gas phase calculation, by considering partial hydration of chloroethenes adsorbed on the Fe surface from the aqueous phase, a distinct difference $(\sim 13 \mathrm{~kJ} / \mathrm{mol})$ in the adsorption energies of PCE and cis-DCE is revealed. Because hydrogen has a more negative hydration enthalpy than chlorine, the more highly halogenated chloroethenes are more strongly adsorbed to the solvated Fe surface. Direct calculations of chloroethene hydration energies in the explicit presence of water using molecular dynamics simulations may be helpful to obtain more accurate hydration energies in the adsorbed state, especially when adsorption on different surface sites such as steps and kinks is considered. 


\subsection{Electronic Property Analysis}

To examine adsorbed configurations in detail, the projected density of states (PDOS) on $d$ orbitals of the relaxed $\mathrm{Fe}(110)-\mathrm{p}(3 \times 2)$ surface atoms was analyzed. Valence electrons in the outermost orbitals of these atoms serve a key role in the adsorption interaction. The PDOS on $d$ orbitals of the Fe surface atoms on which chloroethenes are adsorbed (Figure III-6C, 6D, and 6E) show broader and strongly modified bands ranging from -5 to $-15 \mathrm{eV}$ below the Fermi level compared to that of the clean Fe surface (Figure III-6A). This shows that the molecular orbitals of each chloroethene strongly interact with the $d$ band of the Fe atoms resulting in the mixing band region. To support this, the same PDOS calculation was performed on a relaxed Fe surface, with intact PCE placed 5 $\AA$ above the surface to prevent it from interacting with the surface (Figure III-6B). The PDOS of the $d$ orbital of the Fe surface with the non-interactive PCE is almost unchanged compared with the PDOS of the clean Fe surface (Figure III-6A), indicating little electronic interaction between surface Fe atoms and the far-removed PCE. Thus, the interaction between the chloroethene molecular orbitals and the $d$ band of the relaxed $\mathrm{Fe}(110)-\mathrm{p}(3 \times 2)$ surface atoms is responsible for the mixing band region observed in the PDOS between -5 and -15 eV below the Fermi level. 


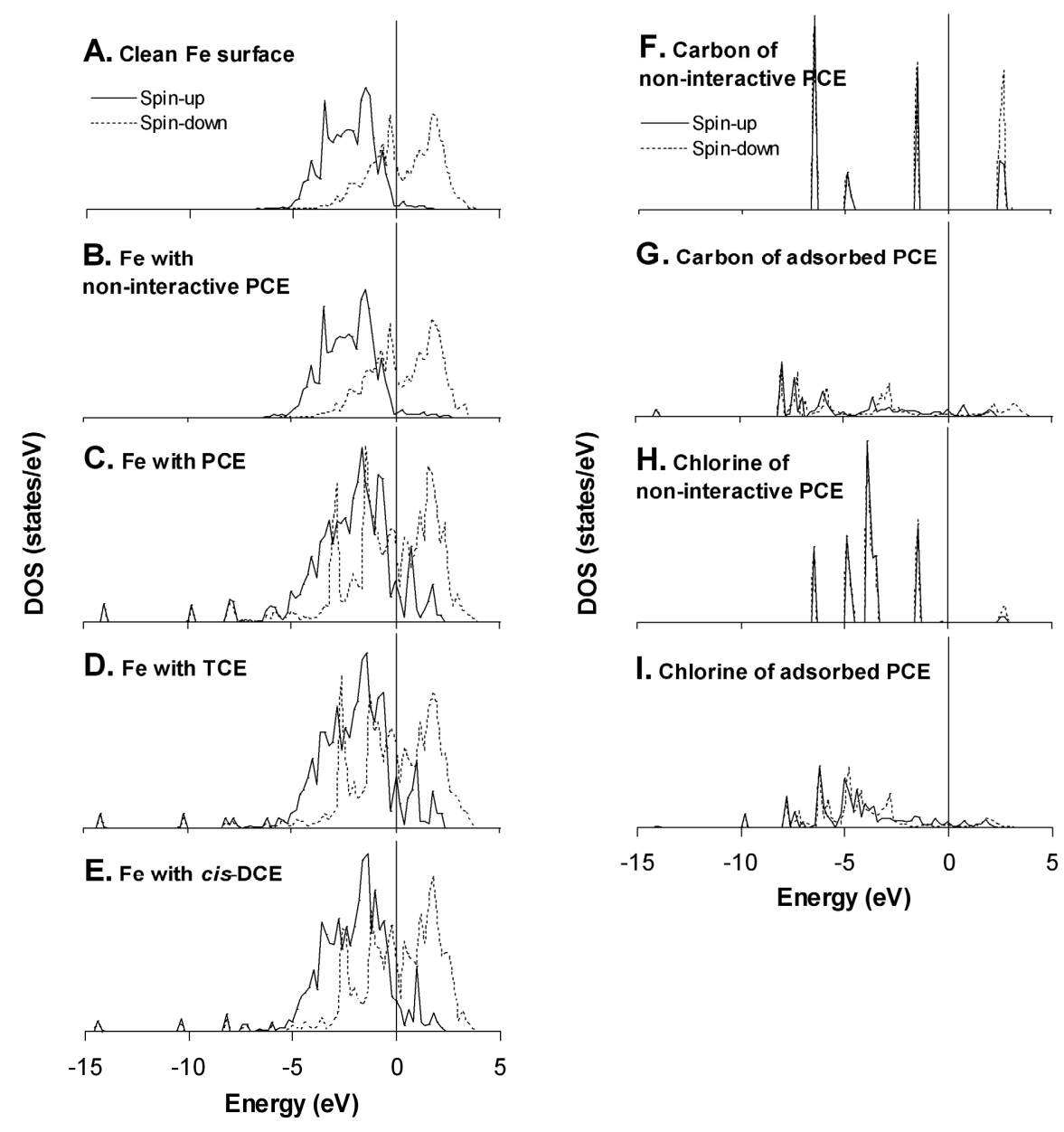

Figure III-6. PDOS on $d$ orbitals of the relaxed Fe(110)-p $(3 \times 2)$ surface (A to E), and $p_{z}$ orbitals of carbon and chlorine atoms of PCE (F to I). (A) Clean iron surface with no adsorbates. (B) Fe surface with PCE molecule $5 \AA$ above the surface (i.e., "noninteractive" PCE). (C) Fe surface with adsorbed PCE at atop 1 site. (D) Fe surface with adsorbed TCE at atop 1 stie. (E) Fe surface with adsorbed cis-DCE at atop 1 site. (F) Carbon and $(\mathrm{H})$ chlorine of non-interactive PCE. (G) Carbon and (I) chlorine of adsorbed PCE at the atop 1 site. The solid and dotted lines indicate PDOS for spin-up and spindown electrons, respectively. The Fermi energy is denoted by the vertical line at $0 \mathrm{eV}$. 
Strong hybridizations were also detected in the $p_{z}$ orbitals (with the $z$ direction perpendicular to the iron surface) of both carbon and chlorine in PCE adsorbed on the $\mathrm{Fe}$ surface (Figure III-6G and 6I), while those of non-interactive PCE by contrast showed localized sharp peaks (Figure III-6F and $6 \mathrm{H}$ ). This is further evidence of the strong interaction between the chloroethene molecules and the Fe surface, with mixing bands at $-14.0 \mathrm{eV}$ in both Figure III-6C and $6 \mathrm{G}$, and at $-9.8 \mathrm{eV}$ and $-7.8 \mathrm{eV}$ in both Figure III-6C and $6 \mathrm{I}$.

The broad bands of the carbon $p_{z}$ orbital covering the range of Fe $d$ bands represent the weakened $\mathrm{C}=\mathrm{C}$ bond, as expected from the elongated $\mathrm{C}=\mathrm{C}$ bond length (Table III-2). A similar observation in the carbon $p$ band was reported in the adsorption of acetylene on $\mathrm{Fe}(001)$ by Lee and coworkers (45). Strong hybridization could probably be attributed to the open shell $3 d^{6}$ structure of iron that participates much more in bonding than closed shell $d$ structure-atoms (e.g. $4 d^{10}$ of silver and $3 d^{10}$ of copper) (34).

In addition to the PDOS analysis, the highest occupied molecular orbitals (HOMOs) and lowest unoccupied molecular orbitals (LUMOs) of adsorbed PCE, TCE, and cis-DCE at the atop 1 site were analyzed using the cluster model approach (see Computational Methods). Although the localized basis set used in the Gaussian 03 cluster model (27) has limitations to describe electronic properties calculated using the plane-wave basis set, the cluster approach is useful to visualize and interpret the molecular orbitals responsible for the interaction between the adsorbed chloroethenes and the iron surface.

From the adsorbed configurations of chloroethenes on the Fe(110) surface (Figure III-5), hybridized bonding $\pi$-orbitals are shown in the HOMO of PCE and in the LUMOs of TCE and cis-DCE (Figure III-7). The $\pi$-orbitals clearly contribute to hybridized bonding between PCE and the Fe surface; however, the $\pi$-orbitals of TCE and cis-DCE do not appear to participate in bonding. This is most likely because the adsorbed geometries of TCE and cis-DCE were obtained at an early stage of adsorption; the adsorbed configurations can vary depending on the convergence criteria used in the geometry optimization (Figure III-2). Thus, other possible adsorbed configurations of PCE, TCE, and cis-DCE were analyzed to verify the $\pi$-orbitals. Figure III- 8 shows the $\alpha$ spin highest and second-highest occupied molecular orbitals (HOMO and HOMO-1, respectively) of the adsorbed chloroethenes, calculated using the tighter convergence 
criteria for geometry optimization. Although the adsorbed chloroethene geometries in Figure III-8 are almost identical to those shown in Figure III-5, with the tighter convergence criteria the $\mathrm{C}=\mathrm{C}$ chloroethene bond in Figure III- 8 approaches $0.1 \AA$ closer to the iron surface than the $\mathrm{C}=\mathrm{C}$ bond in Figure III-5; and the lengths of the $\mathrm{C}=\mathrm{C}$ and $\mathrm{C}-\mathrm{Cl}$ chloroethene bonds are, on average, $0.02 \AA$ shorter and $0.07 \AA$ longer, respectively, in Figure III-8 than in Figure III-5. The tighter convergence criteria thus yields an adsorbate geometry that is nearer to the Fe surface. Also, the hybridized bonding $\pi$ orbitals shown as the LUMOs of TCE and cis-DCE in Figure III-7 are shifted below their energy levels and represent the HOMOs in Figure III-8. Put another way, when the chloroethene geometry is more proximate to the surface, the $\pi$-orbitals shown in the LUMOs are filled from the transfer of $d$-electrons from the iron surface, hence becoming the HOMOs of the adsorbed TCE and cis-DCE configurations. Thus, hybridized bonding $\pi$-orbitals are responsible for the chloroethene adsorption on the $\mathrm{Fe}(110)$ surface. The $d$ orbital character is also evident in the HOMOs; therefore, the interaction between the chloroethenes and the Fe surface may be characterized as $\pi$ - $d$ orbital hybridization. This interaction can be interpreted in terms of the Dewer-Chatt-Duncanson mechanism, where the filled $\pi$ molecular orbital of an alkene donates electrons to unoccupied valence $d$ orbitals of metals, while other occupied $d$ orbitals back-donate into the empty $\pi^{*}$ antibonding orbital of the adsorbate double bond $(46,47)$. The $\pi$ donation and $\pi$ backdonation are responsible for the increase in the carbon-carbon bond distance upon adsorption that accompanies $s p^{2}-s p^{3}$ hybridization (36). Sivavec and Horney (48) and Orth and Gillham (7) have also proposed that strong $\pi$-bonding between chloroethenes and the iron surface may prevent desorption until dechlorination is complete.

In summary, geometric and the electronic property analyses indicate that the atop 1 adsorbed configurations on the $\mathrm{Fe}(110)$ surface is the likely precursors for subsequent dissociation of chloroethenes on the Fe surface. 

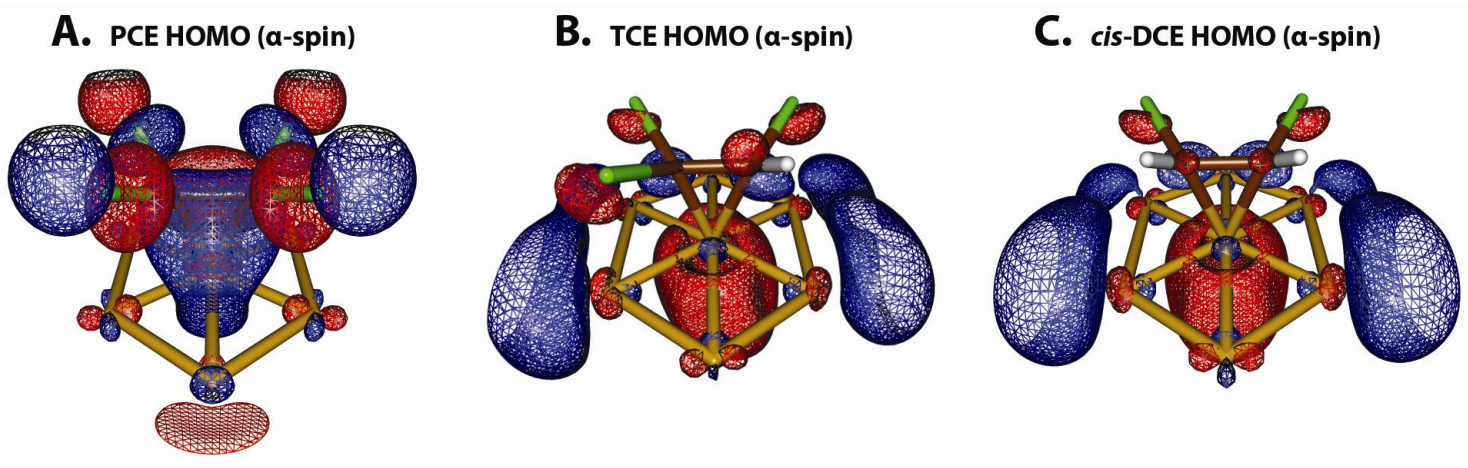

D. PCE LUMO (a-spin)

\section{E. TCE LUMO (a-spin)}
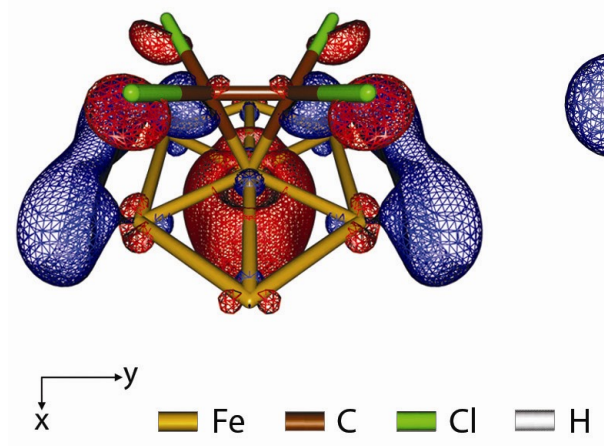

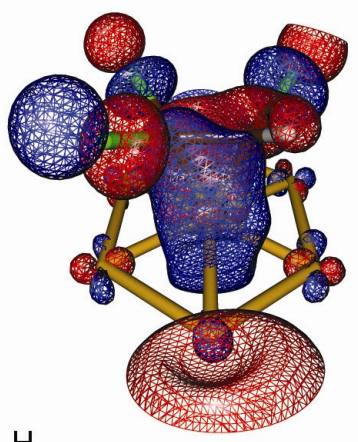

F. cis-DCE LUMO (a-spin)

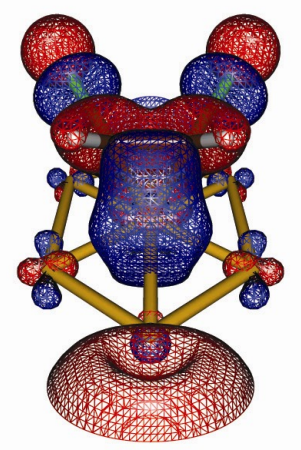

Figure III-7. The $\alpha$-spin highest occupied molecular orbitals (HOMOs: A to C) and lowest unoccupied molecular orbitals (LUMOs: D to F) of adsorbed PCE, TCE, and cisDCE at the atop 1 site of the $\mathrm{Fe}_{7}$ cluster model. The two colors represent the positive and negative portions of the wavefunction.
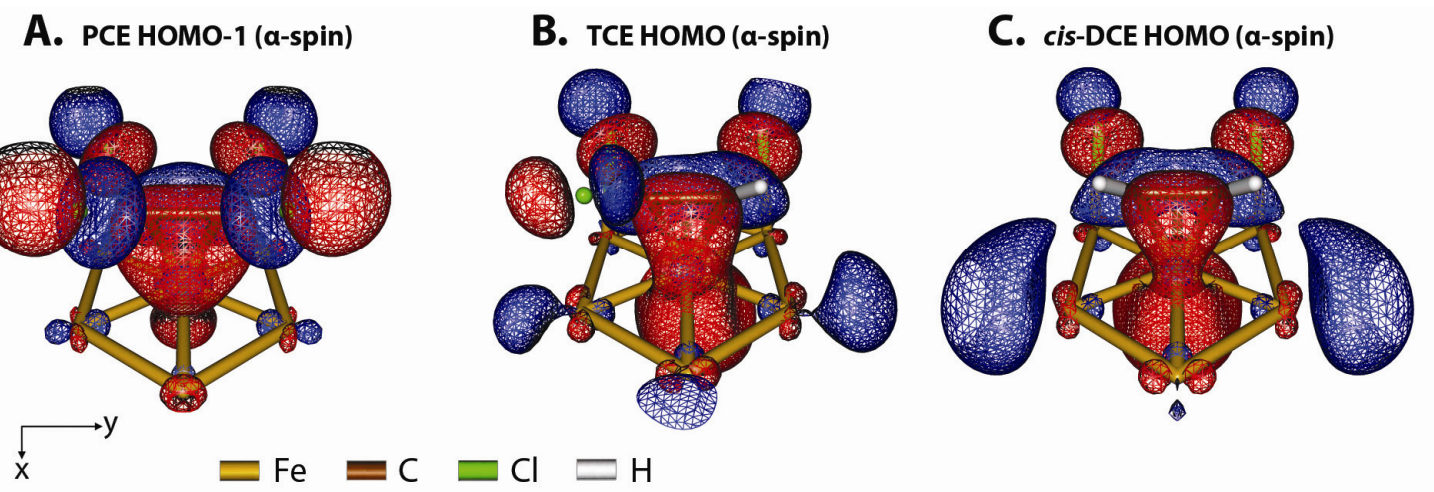

Figure III-8. The $\alpha$-spin second-highest occupied molecular orbitals (HOMO-1) of adsorbed PCE and HOMOs of adsorbed TCE and cis-DCE at the atop 1 site of the $\mathrm{Fe}_{7}$ cluster model. The two colors distinguish the positive and negative portions of the wavefunction. 


\section{References}

(1) Doherty, R. E., A history of the production and use of carbon tetrachloride, tetrachloroethylene, trichloroethylene and 1,1,1-trichloroethane in the United States: Part 1 - Historical background; Carbon tetrachloride and tetrachloroethylene. Environ. Forensics 2000, 1 (2), 69-81.

(2) Doherty, R. E., A history of the production and use of carbon tetrachloride, tetrachloroethylene, trichloroethylene and 1,1,1-trichloroethane in the United States: Part 2 - Trichloroethylene and 1,1,1-trichloroethane. Environ. Forensics 2000, 1 (2), 83-93.

(3) 11th Report on Carcinogens; Dept. of Health and Human Services, National Toxicology Program (NTP): 2005.

(4) Parker, B. L.; Gillham, R. W.; Cherry, J. A., Diffusive disappearance of immisciblephase organic liquids in fractured geologic media. Ground Water 1994, 32 (5), 805 820.

(5) Aggarwal, V.; Li, H.; Boyd, S. A.; Teppen, B. J., Enhanced sorption of trichloroethene by smectite clay exchanged with Cs+. Environ. Sci. Technol. 2006, 40 (3), 894-899.

(6) Arnold, W. A.; Roberts, A. L., Pathways and kinetics of chlorinated ethylene and chlorinated acetylene reaction with $\mathrm{Fe}(\mathrm{O})$ particles. Environ. Sci. Technol. 2000, 34 (9), 1794-1805.

(7) Orth, W. S.; Gillham, R. W., Dechlorination of trichloroethene in aqueous solution using Fe-O. Environ. Sci. Technol. 1996, 30 (1), 66-71.

(8) Henderson, A. D.; Demond, A. H., Long-term performance of zero-valent iron permeable reactive barriers: A critical review. Environ. Eng. Sci. 2007, 24 (4), 401423.

(9) Matheson, L. J.; Tratnyek, P. G., Reductive dehalogenation of chlorinated methanes by iron metal. Environ. Sci. Technol. 1994, 28 (12), 2045-2053.

(10) Arnold, W. A.; Roberts, A. L., Pathways of chlorinated ethylene and chlorinated acetylene reaction with $\mathrm{Zn}(0)$. Environ. Sci. Technol. 1998, 32 (19), 3017-3025.

(11) Johnson, T. L.; Scherer, M. M.; Tratnyek, P. G., Kinetics of halogenated organic compound degradation by iron metal. Environ. Sci. Technol. 1996, 30 (8), 26342640.

(12) Scherer, M. M.; Balko, B. A.; Gallagher, D. A.; Tratnyek, P. G., Correlation analysis of rate constants for dechlorination by zero-valent iron. Environ. Sci. Technol. 1998, 32 (19), 3026-3033.

(13) Lee, W. J.; Batchelor, B., Abiotic reductive dechlorination of chlorinated ethylenes by iron-bearing phyllosilicates. Chemosphere 2004, 56 (10), 999-1009.

(14) Lee, W.; Batchelor, B., Abiotic reductive dechlorination of chlorinated ethylenes by iron-bearing soil minerals. 1. Pyrite and magnetite. Environ. Sci. Technol. 2002, 36 (23), 5147-5154.

(15) Lee, W.; Batchelor, B., Abiotic, reductive dechlorination of chlorinated ethylenes by iron-bearing soil minerals. 2. Green rust. Environ. Sci. Technol. 2002, 36 (24), 5348-5354. 
(16) Lowry, G. V.; Reinhard, M., Hydrodehalogenation of 1-to 3-carbon halogenated organic compounds in water using a palladium catalyst and hydrogen gas. Environ. Sci. Technol. 1999, 33 (11), 1905-1910.

(17) Butler, E. C.; Hayes, K. F., Kinetics of the transformation of trichloroethylene and tetrachloroethylene by iron sulfide. Environ. Sci. Technol. 1999, 33 (12), 2021-2027.

(18) Burris, D. R.; Campbell, T. J.; Manoranjan, V. S., Sorption of trichloroethylene and tetrachloroethylene in a batch reactive metallic iron-water system. Environ. Sci. Technol. 1995, 29 (11), 2850-2855.

(19) Valcarcel, A.; Clotet, A.; Ricart, J. M.; Illas, F., Comparative theoretical study of the structure and bonding of propyne on the $\operatorname{Pt}(111)$ and $\operatorname{Pd}(111)$ surfaces. Chem. Phys. 2005, 309, 33-39.

(20) Vanderbilt, D., Soft self-consistent pseudopotentials in a generalized eigenvalue formalism. Phys. Rev. B 1990, 41 (11), 7892-7895.

(21) Perdew, J. P.; Burke, K.; Ernzerhof, M., Generalized gradient approximation made simple. Phy. Rev. Lett. 1996, 77 (18), 3865-3868.

(22) Baroni, S.; Dal Corso, A.; de Gironcoli, S.; Giannozzi, P. Quantum ESPRESSO: opEn-Source Package for Research in Electronic Structure, Simulation, and Optimization, v. 3.2; http://www.pwscf.org/: 2007.

(23) Monkhorst, H. J.; Pack, J. D., Special points for brillouin-zone integrations. Phys. Rev. B 1976, 13 (12), 5188-5192.

(24) Jiang, D. E.; Carter, E. A., Adsorption and diffusion energetics of hydrogen atoms on Fe(110) from first principles. Surf. Sci. 2003, 547 (1-2), 85-98.

(25) Jiang, D. E.; Carter, E. A., Adsorption and dissociation of $\mathrm{CO}$ on $\mathrm{Fe}(110)$ from first principles. Surf. Sci. 2004, 570 (3), 167-177.

(26) Kokalj, A., Computer graphics and graphical user interfaces as tools in simulations of matter at the atomic scale. Comput. Mater. Sci. 2003, 28 (2), 155-168.

(27) Frisch, M. J., et al. Gaussian 03, Revision C.02; Gaussian, Inc.: Wallingford CT, 2004.

(28) Schaftenaar, G.; Noordik, J. H., Molden: a pre- and post-processing program for molecular and electronic structures. J. Comput. Aided Mol. Des. 2000, 14 (2), 123134.

(29) Miertus, S.; Tomasi, J., Approximate evaluations of the electrostatic free-energy and internal energy changes in solution processes. Chem. Phys. 1982, 65 (2), 239245.

(30) Soon, A.; Todorova, M.; Delley, B.; Stampfl, C., Oxygen adsorption and stability of surface oxides on $\mathrm{Cu}(111)$ : A first-principles investigation. Phys. Rev. B 2006, 73 (16).

(31) Shih, H. D.; Jona, F.; Bardi, U.; Marcus, P. M., The atomic structure of Fe $\{110\} . J$. Phys. C Solid State 1980, 13 (19), 3801-3808.

(32) Spencer, M. J. S.; Hung, A.; Snook, I. K.; Yarovsky, I., Density functional theory study of the relaxation and energy of iron surfaces. Surf. Sci. 2002, 513 (2), 389398.

(33) Barbosa, L.; Loffreda, D.; Sautet, P., Chemisorption of trichloroethene on the PdCu alloy (110) surface: A periodical density functional study. Langmuir 2002, 18 (7), 2625-2635. 
(34) Bernardo, C.; Gomes, J., The adsorption of ethylene on the (110) surfaces of copper, silver and platinum: a DFT study. J. Mol. Struc.-Theochem. 2002, 582, 159-169.

(35) Kokalj, A.; Dal Corso, A.; de Gironcoli, S.; Baroni, S., DFT study of a weakly pibonded $\mathrm{C}_{2} \mathrm{H}_{4}$ on oxygen-covered $\mathrm{Ag}(100)$. J. Phys. Chem. B 2006, 110 (1), 367-376.

(36) Itoh, K.; Kiyohara, T.; Shinohara, H.; Ohe, C.; Kawamura, Y.; Nakai, H., DFT calculation analysis of the infrared spectra of ethylene adsorbed on $\mathrm{Cu}(110)$, Pd(110), and Ag(110). J. Phys. Chem. B 2002, 106 (41), 10714-10721.

(37) Watson, G. W.; Wells, R. P. K.; Willock, D. J.; Hutchings, G. J., Density functional theory calculations on the interaction of ethene with the $\{111\}$ surface of platinum. J. Phys. Chem. B 2000, 104 (27), 6439-6446.

(38) Bocquet, M. L.; Sautet, P.; Cerda, J.; Carlisle, C. I.; Webb, M. J.; King, D. A., Specific ethene surface activation on silver oxide covered $\mathrm{Ag}\{111\}$ from the interplay of STM experiment and theory. J. Am. Chem. Soc. 2003, 125 (10), 31193125.

(39) Zheng, T.; Stacchiola, D.; Poon, H. C.; Saldin, D. K.; Tysoe, W. T., Determination of the structure of disordered overlayers of ethylene on clean and hydrogen-covered Pd(111) by low-energy electron diffraction. Surf. Sci. 2004, 564 (1-3), 71-78.

(40) Zhang, N. L.; Luo, J.; Blowers, P.; Farrell, J., Understanding trichloroethylene chemisorption to iron surfaces using density functional theory. Environ. Sci. Technol. 2008, 42 (6), 2015-2020.

(41) Barbosa, L.; Sautet, P., Trichloroethene dechlorination reactions on the $\mathrm{PdCu}(110)$ alloy surface: A periodical density functional theory study of the mechanism. $J$. Catal. 2002, 207 (1), 127-138.

(42) Desai, S. K.; Pallassana, V.; Neurock, M., A periodic density functional theory analysis of the effect of water molecules on deprotonation of acetic acid over Pd(III). J. Phys. Chem. B 2001, 105 (38), 9171-9182.

(43) Zolotoy, N. B., Estimate of the enthalpy change of proton hydration in water. Dokl. Phys. Chem. 2006, 406, 30-32.

(44) Becker, U.; Risthaus, P.; Bosbach, D.; Putnis, A., Selective attachment of monovalent background electrolyte ions and growth inhibitors to polar steps on sulfates as studied by molecular simulations and AFM observations. Mol. Simulat. 2002, 28 (6-7), 607-632.

(45) Lee, G. D.; Han, S. W.; Yu, J. J.; Ihm, J., Catalytic decomposition of acetylene on $\mathrm{Fe}(001)$ : A first-principles study. Phys. Rev. B 2002, 66 (8).

(46) Dewar, M. J. S.; Ford, G. P., Relationship between olefinic pi-complexes and 3membered rings. J. Am. Chem. Soc. 1979, 101 (4), 783-791.

(47) Zaera, F., An organometallic guide to the chemistry of hydrocarbon moieties on transition metal surfaces. Chem. Rev. 1995, 95 (8), 2651-2693.

(48) Sivavec, T. M.; Horney, D. P., Reductive dechlorination of chlorinated ethenes by iron metal; 208th National Meeting, American Chemical Society, Anaheim, CA; Environmental Division Extended Abstracts; . American Chemical Society: Washington, DC, 1995; p 695. 


\section{CHAPTER IV DISSOCIATION OF CHLOROETHENES ON FE(110)}

\section{Introduction}

In the presence of zerovalent iron, reductive dechlorination of chloroethenes occurs through $\beta$-elimination, hydrogenolysis, $\alpha$-elimination, and hydrogenation (1-4). Reductive elimination removes two halide ions whereas hydrogenolysis replaces a halogen with a hydrogen atom. Both reductive elimination and hydrogenolysis are accompanied by a net transfer of two electrons (5). Reduction of PCE, TCE, cis-DCE and trans-DCE to acetylene by reductive $\beta$-elimination is strongly kinetically favored over reduction to $\mathrm{VC}$ and ethene by hydrogenolysis $(2-4,6)$. Arnold and Roberts (4) reported that the $\beta$-elimination pathway accounts for $87 \%$ of PCE reduction, $97 \%$ of TCE reduction, 94\% of cis-DCE reduction, and 99\% of trans-DCE reduction, yielding dichloroacetylene from PCE, chloroacetylene from TCE, and acetylene from cis- and trans-DCE.

In the studies reported herein, density functional theory (DFT) methods were applied to investigate the relative reactivity of chloroethenes on the iron surface. In our previous study of chlorethene adsorption on the $\mathrm{Fe}(110)$ surface (Chapter III), the thermodynamically favorable adsorption sites for PCE, TCE, and cis-DCE were determined as a first step toward elucidating the effect of chlorination on the relative reactivity of chloroethenes on the iron surface. Using the adsorbed configurations of the chloroethenes, this study determines the activation energies of the chloroethenes on the iron surface, based on assumed dechlorination pathways governed by reductive $\beta$ elimination. The activation energies are converted into dechlorination reaction rate constants using the vibrational frequencies of the chloroethene products and transition states along the reaction pathways. We also report the electronic properties of chloroethenes adsorbed and dissociated on the Fe(110) surface. Not only does this study close knowledge gaps in our understanding of chloroethene dechlorination pathways, but 
it also enables confirmation or rejection of hypotheses concerning the relative reactivity of chloroethenes on zerovalent iron.

\section{Computational Methods}

All electronic structure calculations were performed using periodic density functional theory and the ultrasoft Vanderbilt pseudopotential method (7) within the generalized gradient approximation (GGA) by Perdew, Burke, and Ernzerhof (PBE) (8) for the exchange-correlation functional, as implemented in the Plane-Wave SelfConsistent Field (PWscf) code in the Quantum-ESPRESSO open-source distribution (9). Kinetic-energy cutoffs for wavefunctions and for charge density and potential were 340.1 eV (25 Ryd) and $2449.0 \mathrm{eV}$ (180 Ryd), respectively; this converges the total energy to within $0.01 \mathrm{eV} /$ atom. The Monkhorst-Pack scheme (10) was used for $k$-point sampling. Using a $10 \times 10 \times 10 k$-point mesh, the following physical properties were calculated for ferromagnetic bcc iron: equilibrium lattice constant $a_{0}=2.8606 \AA$; bulk modulus $B=167$ GPa; and magnetic moment $M=2.45 \mu_{\mathrm{B}}$. These results are in good agreement with experimental results $(11)$ for ferromagnetic bcc iron: $a_{0}=2.8664 \AA, B=168 \mathrm{GPa}, M=$ $2.22 \mu_{\mathrm{B}}$

$\mathrm{Fe}(110)$ is the closest-packed surface of body-centered cubic (bcc) iron. It is essentially bulk-terminated, with very little relaxation and no reconstruction $(12) . \mathrm{p}(4 \times 2)$ supercells of $\mathrm{Fe}(110)$ were modeled using periodic three-layer slabs with chloroethenes (PCE, TCE or cis-DCE) adsorbed on both sides of the slabs. This double-sided adsorption model minimizes the dipole moment perpendicular to the surface that is produced by charge rearrangement on the surface due to adsorption. The outermost layers (surface layers) of the slab were allowed to relax, whereas the center layer (below the surface) was kept rigid to represent the underlying bulk iron. The coverage of each adsorbate molecule was 0.063 monolayers $(\mathrm{ML})$ for the $\mathrm{p}(4 \times 2)$ supercells. The slabs were separated from their periodic images in the $z$-direction by a vacuum space of $16 \AA$. Brillouin-zone integrations were performed on a $2 \times 3 \times 1$ Monkhorst-Pack grid of $k$-points for $\mathrm{Fe}(110)$ supercells as used in our previous study of chloroethene adsorption on $\mathrm{Fe}(110)$ (Chapter III). 


\subsection{Activation energy calculation using climbing image nudged elastic band (CI- NEB) method}

The activation energies for dechlorination of chloroethenes were computed for the $\beta$-elimination pathways using the CI-NEB method. This method is known to effectively locate the minimum energy paths (MEPs) and the transition states of adsorbate molecules on the transition metal surface $(12,13)$. Once the initial and the final state along a reaction path are known, intermediate configurations are determined by linearly interpolating between the initial and final configurations. The interpolated configurations are connected by springs and relaxed simultaneously to the MEP, through which the highest-energy configuration climbs uphill to the saddle point $(12,13)$. Two sets of forces are projected to the connected image band and minimized to obtain the MEP; one is the set of potential forces acting perpendicular to the band, and the other is the set of spring forces acting along the band (14).

Among the adsorption sites examined in our previous study (Figure III-1 in Chapter III), the atop site, where the chloroethene $\mathrm{C}=\mathrm{C}$ bond straddles a surface iron atom, was determined to be the most energetically favorable site for the adsorption of PCE, TCE and cis-DCE. The adsorbed configurations were used as the initial images for CI-NEB analysis of chloroethene dissociation. It is known that dissociation of chloroethenes occurs mainly through a $\beta$-elimination mechanism in which two chlorine atoms are simultaneously detached from the carbon-carbon $(\mathrm{C}=\mathrm{C})$ backbone (4). Experimentally, it is difficult to observe the sequential detachment of the two chlorine atoms from the adsorbed chloroethene species during the reduction reaction. However, computational approaches using density functional theory allow us to investigate the detachment of each chlorine atom from the chloroethene $\mathrm{C}=\mathrm{C}$ bond. It is also generally known that the activation energy for removing one chlorine atom is energetically lower than that for removal of two chlorines. Thus, it is reasonable to assume that chloroethene dissociation proceeds through the following two steps. First, two chlorine atoms consecutively detach from the $\mathrm{C}=\mathrm{C}$ backbone of the fixed chloroethene molecule and adsorb onto the $\mathrm{Fe}$

surface. Second, the dechlorinated molecular species $(\mathrm{Cl}-\mathrm{C} \equiv \mathrm{C}-\mathrm{Cl}, \mathrm{Cl}-\mathrm{C} \equiv \mathrm{C}-\mathrm{H}$, or $\mathrm{H}-\mathrm{C} \equiv \mathrm{C}-\mathrm{H})$ rearranges on the surface so as to optimize its adsorbed configuration. 
A total of four step images, including the initial and final images, were employed to calculate the activation energy of the reaction pathway (Figure IV-1). The most favorable site for adsorption of a chlorine atom on the $\mathrm{Fe}(110)$ surface has been found to be the hollow site surrounded by three Fe atoms, as shown for TCE dechlorination in Figure IV1D. Hence, this geometry was used as the final image in the CI-NEB analysis of the putative chloroethene dissociation pathway. The activation energies were calculated using a fully-relaxed $\mathrm{Fe}(110)$ surface. The activation energy $\left(E_{a c t}\right)$ per chloroethene molecule is calculated as

$$
E_{a c t}=\left(E_{T S}-E_{R T}\right) / N_{\text {adsorbate }}
$$

where $N_{\text {adsorbate }}$ is the number of chloroethene molecules in the model system, and $E_{T S}$ and $E_{R T}$ represent the total energies of the transition state and reactant, respectively. Figure IV-2 shows possible dechlorination pathways in accordance with the assumed dissociated states. The optimized structures were visualized using the XCrySDen graphical package (15).
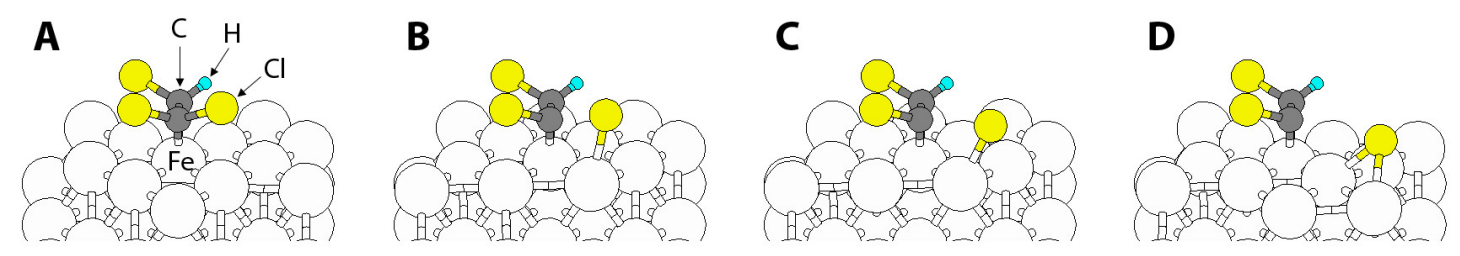

Figure IV-1. $\beta$-elimination pathway for dissociation of the first chlorine from TCE, showing the four images used in the reaction path analysis. (A) Adsorbed TCE, (B) $2^{\text {nd }}$ image, (C) $3^{\text {rd }}$ image, and (D) $1^{\text {st }} \mathrm{Cl}$-dissociated TCE. 


\subsection{Reaction rate constant calculation using frequency}

Although the calculated activation energies may suggest the relative reactivity of chloroethene reduction along competing pathways, the reaction kinetics are not determined by the activation energies alone (16). The kinetics of a single reaction step can be described with an Arrhenius equation. The dechlorination rate constant $\left(k_{d e C l}\right)$ at a temperature $T$ can be evaluated as shown by Barbosa and Sautet (17):

$$
k_{d e C l}=\frac{k_{B} T}{h} \frac{p f^{T s}}{p f^{R s}} \exp \left(-\frac{E_{a}}{R T}\right)
$$

where $k_{B}$ is Boltzmann's constant, $h$ is Planck's constant, $R$ is the gas constant, and $E_{a}$ is the activation energy per mole between the reactants and the transition state. In this study, $E_{a}$ is not corrected for the zero-point energy vibrations (ZPE). $p f^{R s}$ and $p f^{T s}$ are the partition functions of the reactant and transition states, respectively. By assuming the $\mathrm{Fe}$ surface is rigid, the partition functions can be expressed in terms of various vibration modes and calculated if the wavenumbers $(\widetilde{v})$ of the adsorbates are given (17-19).

$$
\begin{gathered}
p f=\prod_{i} \frac{\exp \left(+\frac{1}{2} \frac{h v_{i}}{k_{B} T}\right)}{1-\exp \left(\frac{h v_{i}}{k_{B} T}\right)} \\
\frac{h v_{i}}{k_{B} T}=4.8 \times 10^{-3}\left(\frac{\widetilde{v}_{i}}{1 \mathrm{~cm}^{-1}}\right)\left(\frac{300 K}{T}\right)
\end{gathered}
$$

The frequency calculations were performed using the cluster model approach as described in Chapter III. The GGA of PBE for the exchange-correlation functional was used with the Los Alamos LanL2DZ double-zeta $(\zeta)$ basis set. The optimized coordinates of the reactant and transition states, along with those of the neighboring Fe surface atoms ( $\mathrm{Fe}_{7}$ cluster) were transferred to the Gaussian 03 program package (20) to calculate the frequencies of the adsorbed chloroethenes. The $\mathrm{Fe}_{7}$ cluster model effectively represents the electronic properties of adsorbed chloroethenes, as discussed in Chapter III. The 
relaxed Fe surface, atoms optimized in the plane-wave code, were kept frozen in the frequency calculations using the cluster models described by Barbosa and Sautet (17). 


\section{Results and Discussion}

\subsection{Activation energies of PCE, TCE, and cis-DCE on Fe(110)}

Figure IV-2 shows the calculated activation energies. It is observed that chloroethenes with a higher number of chlorine atoms have lower activation energies than those with fewer chlorine atoms. Each dechlorination pathway has a different ratelimiting step. Activation energies shown in bold denote the most favorable dissociation pathways at each dechlorination step. In all cases, loss of chlorine is a favorable decomposition for PCE, TCE, and cis-DCE on Fe(110). This is the same result as shown in the experimental study of chloroethene reaction on $\mathrm{Cu}(100)$ (21). All dissociation reactions of the chloroethenes on $\mathrm{Fe}(110)$ are strongly exothermic and the activation energies are relatively small $(<25 \mathrm{~kJ} / \mathrm{mol})$, which agrees with DFT computations of TCE dechlorination on $\mathrm{PdCu}(110)$ alloy surface (17). The activation energies for $\mathrm{C}-\mathrm{Cl}$ bond scissions of PCE, TCE, and cis-DCE on Fe(110) are 3-7\% of the gas-phase bond dissociation enthalpies of PCE (334.6 kJ/mol (22)) and TCE (357.4 kJ/mol (22)). This is comparable to the activation energies for $\mathrm{C}-\mathrm{Cl}$ bond scission of alkyl halides and multiply-chlorinated ethanes on copper surfaces, which were found to be $12-20 \%$ of the gas-phase bond dissociation energy $(23,24)$. Zerovalent iron has a lower redox potential $\left(E_{H}{ }^{\circ}=-0.44 \mathrm{~V}\right)$ compared with copper $\left(E_{H}{ }^{\circ}=+0.34 \mathrm{~V}\right)$. Hence, iron is a stronger reductant than copper and has lower activation energies than copper for reducing chlorinated compounds. As a dopant metal to accelerate dechlorination of PCE on zerovalent silicon $(\mathrm{Si})$, iron increased the dechlorination rate relative to zerovalent $\mathrm{Si}$ by $50 \%$, whereas copper as a dopant lowered the dechlorination rate by $45 \%$ (25). The lower activation energies for dechlorination on iron than on copper are most likely due to the partially occupied, open shell $3 d^{6}$ structure of iron, which participates much more in bond formation and dechlorination than the fully occupied, closed shell $3 d^{10}$ structure of copper (26). 

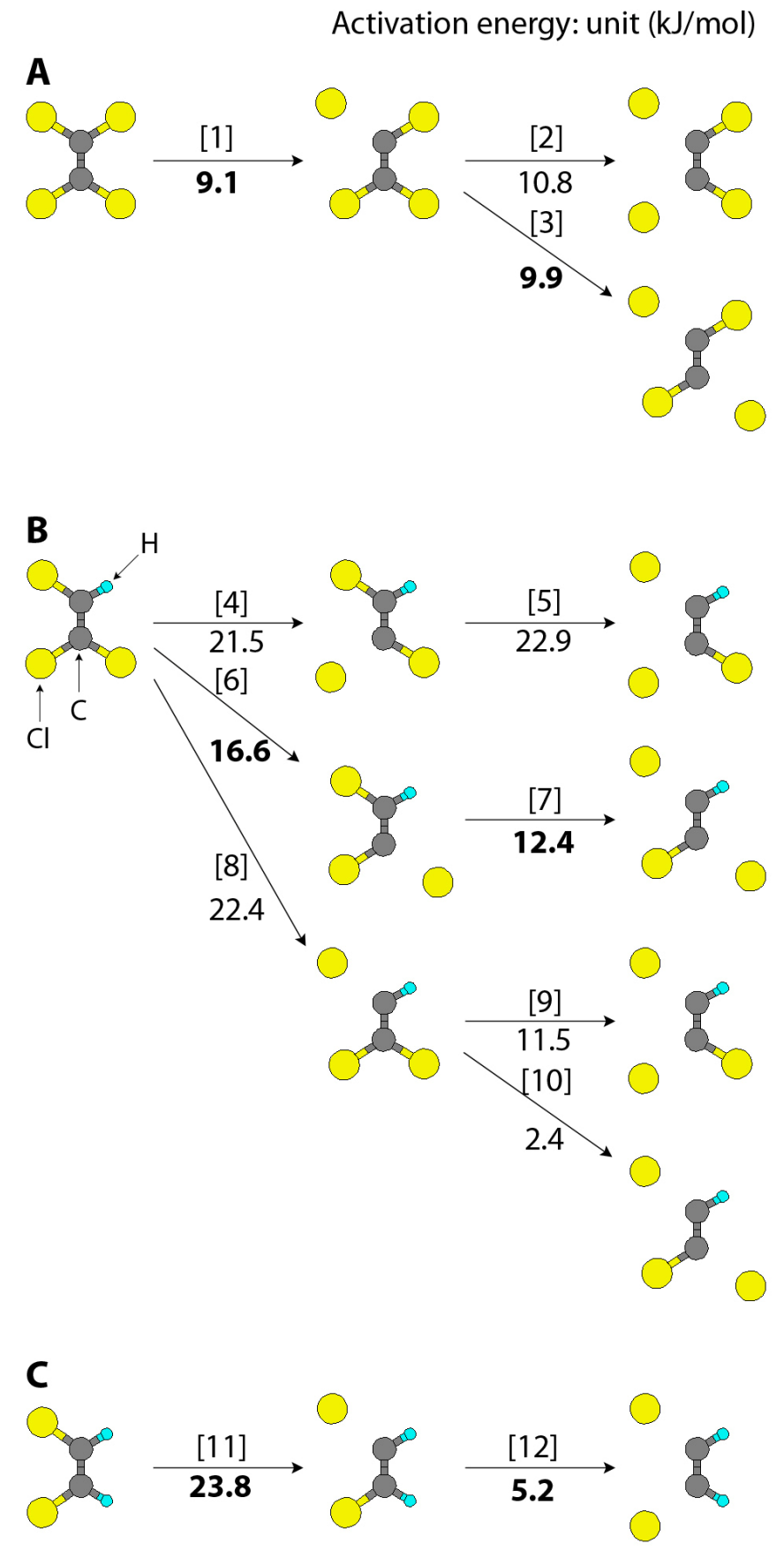

Figure IV-2. Chloroethene dissociation pathways and activation energies for (A) PCE, (B) TCE, and (C) cis-DCE. 


\subsection{Dechlorination of TCE}

The elongated $\mathrm{C}-\mathrm{Cl}$ bond distances for adsorbed TCE on Fe(110) (Table III-2 in Chapter III) are different from one another due to the asymmetry of the adsorbate. This asymmetry allows four possible dechlorination pathways, according to the stepwise removal assumed in this study (Figure IV-2). Barriers for the first and second $\mathrm{C}-\mathrm{Cl}$ cleavages of TCE are relatively small (less than $23 \mathrm{~kJ} / \mathrm{mol}$ ). A similar result has been observed in TCE dissociation on $\mathrm{PdCu}(110)$ (17). Among the possible pathways for TCE dissociation on $\mathrm{Fe}(110)$ shown in Figure IV-2, $\mathrm{C}-\mathrm{Cl}$ bond dissociation from the $\mathrm{CCl}_{2}$ group (Pathway [6]: activation energy $=16.6 \mathrm{~kJ} / \mathrm{mol}$ ) is more favorable than that from $\mathrm{CHCl}$ group (Pathway [8]: activation energy $=22.4 \mathrm{~kJ} / \mathrm{mol}$ ). This is consistent with Barbosa and Sautet's finding (17) that the activation energies of $\mathrm{C}-\mathrm{Cl}$ bond dissociation of TCE on $\mathrm{PdCu}(110)$ from the $\mathrm{CCl}_{2}$ group and from the $\mathrm{CHCl}$ group are $8 \mathrm{~kJ} / \mathrm{mol}$ and 25 $\mathrm{kJ} / \mathrm{mol}$, respectively. In addition, Yang et al. (23) reported that $\mathrm{C}-\mathrm{Cl}$ bond dissociation is more facile in $\mathrm{CCl}_{2}$ groups than in $\mathrm{CCl}$ groups of multiply-chlorinated hydrocarbons on $\mathrm{Cu}(100)$. He et al. (27) observed that $\mathrm{Cl}$ dissociation from the $\mathrm{CCl}_{2}$ group in iso-DCE occurs more readily than from the $\mathrm{CHCl}$ group in the cis- and trans-DCE isomers on the $\operatorname{Si}(111) 7 \times 7$ surface. Of the two chlorine atoms in the $\mathrm{CCl}_{2}$ group of adsorbed TCE on $\mathrm{Fe}(110)$, the chlorine nearer to the hydrogen atom has a more elongated $\mathrm{C}-\mathrm{Cl}$ bond (1.850 $\AA$, from Table III-2 in Chapter III). and removal of this chlorine has the lowest activation energy $(16.6 \mathrm{~kJ} / \mathrm{mol})$ among the three possible pathways for dissociation of the first chlorine from TCE.

Figure IV-3 shows the energy diagram for the most energetically favored dissociation pathway for TCE on Fe(110) (Pathway [6] and [7] in Figure IV-2). Transition states for both the first and the second $\mathrm{Cl}$ dissociations were obtained from the NEB method and are shown in Figure IV-3. It was found that the transition states more closely resemble the initial structures (reactants) than the final structures (products), suggesting that the TCE dissociation reactions have an early transition state as reported in previous studies $(17,28)$. The dissociation of two chlorines from TCE is strongly exothermic and releases $485 \mathrm{~kJ} / \mathrm{mol}$. The second $\mathrm{Cl}$ dissociation has a lower activation energy than the first, indicating that the first $\mathrm{Cl}$ dissociation is the rate-limiting step. One may note that the dissociated $\mathrm{Cl}$ atoms are located at hollow sites surrounded by three $\mathrm{Fe}$ 
atoms on the $\mathrm{Fe}(110)$ surface. Once two chlorine atoms were detached from the $\mathrm{C}=\mathrm{C}$ backbone of TCE, the geometry of the product molecule, chloroacetylene $(\mathrm{Cl}-\mathrm{C} \equiv \mathrm{C}-\mathrm{H})$, was optimized on $\mathrm{Fe}(110)$. Chloroacetylene moved from an atop site to a long bridge site where the $\mathrm{C} \equiv \mathrm{C}$ bond bridge spans two iron atoms separated by the calculated equilibrium lattice constant of $2.8606 \AA$. The chloroacetylene $\mathrm{C}-\mathrm{Cl}$ and $\mathrm{C}-\mathrm{H}$ bonds bend away from the surface about $60^{\circ}$ and $59^{\circ}$, respectively. This geometry is consistent with a previous theoretical study of acetylene adsorption on the long bridge site of $\mathrm{Fe}(110)$ (29). The chloroacetylene $\mathrm{C} \equiv \mathrm{C}, \mathrm{C}-\mathrm{Cl}$, and $\mathrm{C}-\mathrm{H}$ bond lengths are $1.399 \AA, 1.783 \AA$, and $1.103 \AA$, respectively. The $\mathrm{C} \equiv \mathrm{C}$ bond stretches about $0.2 \AA$ compared to the experimental gasphase acetylene geometry (30), consistent with previous studies of acetylene adsorption on $\mathrm{Fe}(110)$ (29). Considering that the $s p^{2}$ hybridized $\mathrm{C}=\mathrm{C}$ bond length is $1.388 \AA$, and the $s p^{3}$ hybridized $\mathrm{C}-\mathrm{C}$ bond length is $1.537 \AA$ (31), the adsorbed configuration of chloroacetylene suggests that both of its carbon atom orbitals are still close to a $s p^{2}$ hybridization. Barbosa and Sautet (17) also reported that the carbon atoms of chloroacetylene-like intermediates in the dechlorination reaction path of TCE on $\mathrm{PdCu}(110)$ have a resemblance to $s p^{2}$ hybridization. 


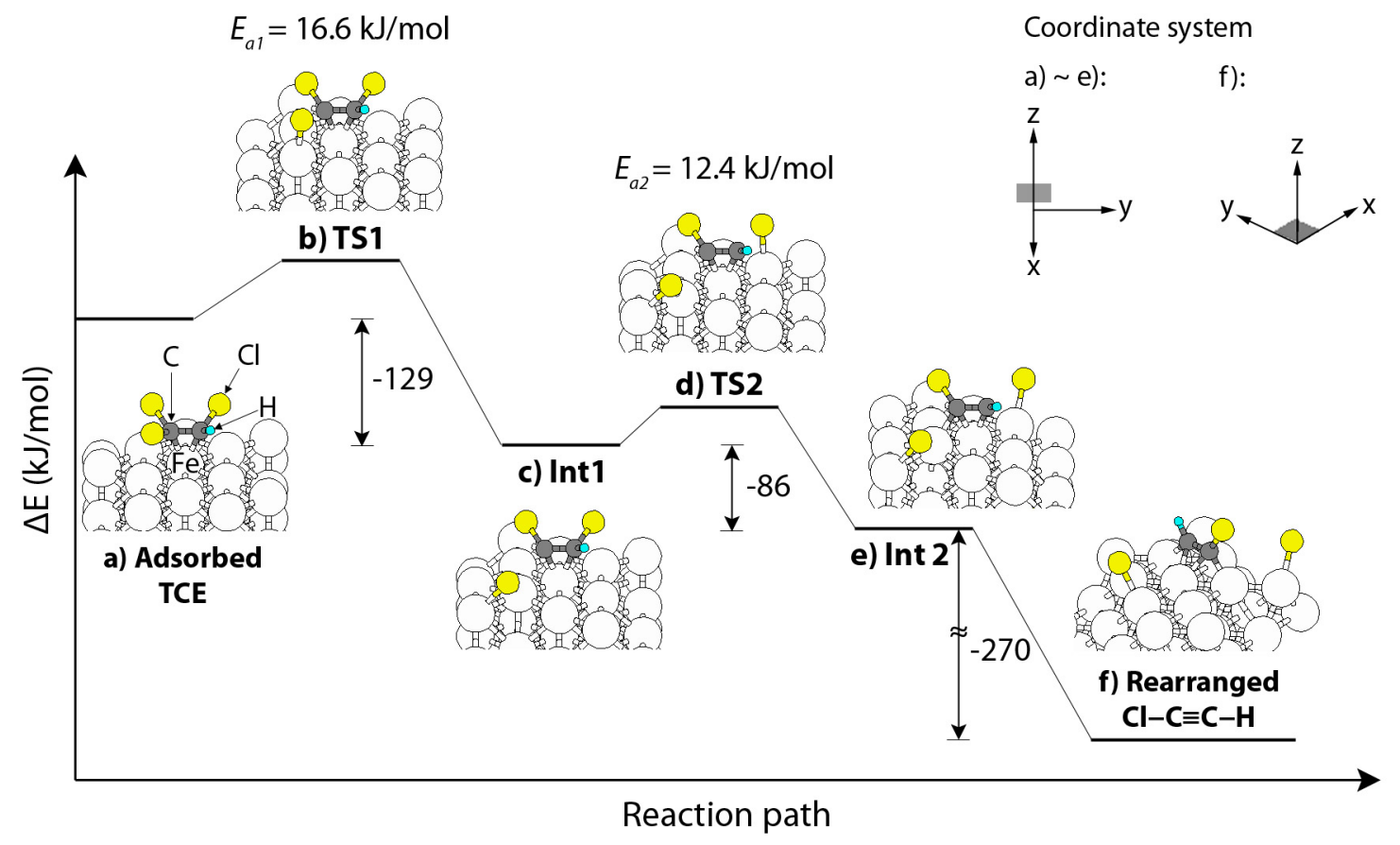

Figure IV-3. Energy diagram for TCE dechlorination (Pathway [6] and [7] in Figure IV2). TS and Int denote transition states and intermediates, respectively. The energy differences between the adsorbed reactant, intermediates, and product are reported in $\mathrm{kJ} / \mathrm{mol}$. The viewing angle and orientation used for the coordinate system in the images is shown in the top right-hand corner.

Most TCE reduction (97\%) occurs through $\beta$-elimination to chloroacetylene (4). The remaining 3\% involves TCE reduction to dichloroethenes (cis-, trans-, and 1,1DCE). Pathways [4], [6], and [8] in Figure IV-2 can be used as models for the formation of trans-DCE, cis-DCE, and 1,1-DCE, respectively from TCE through hydrogenolysis reactions in which the dissociated chlorine atom is replaced by hydrogen atom. Based on the activation energies only, cis-DCE formation would be favored over other DCE isomers due to the lowest activation energy of Pathway [6]. This finding is consistent with Arnold and Roberts's experimental measurement (4) of cis-DCE concentration that was observed to be 1.3-1.4 times higher than the concentrations of other DCE species produced from TCE reduction on zerovalent iron.

The calculated activation energy of TCE on Fe(110) ranges between 16.6 and 22.9 $\mathrm{kJ} / \mathrm{mol}$. Compared to previously reported experimental measurements of 32.2 to 39.4 $\mathrm{kJ} / \mathrm{mol}$ (32) for the TCE activation energy on zerovalent iron, there is disparity between 
our values and the experimental measurements. This disparity can, in part, be explained by experimental conditions used in the experimental calculations. The experimental measurements (32) represent overall activation energy for dechlorination of TCE to the final products $c i s$-DCE, ethane, and ethane. No chloroacetylene was detected in the final products, which indicates no evidence for $\beta$-elimination in the experiments. Considering that our activation energy represents activation energy for the first chlorine dissociation of TCE according to the $\beta$-elimination mechanism, the experimental results may not be directly compared with our theoretical results. Considering all activation energies at all dissociation steps of TCE to the final products in this study, the theoretically calculated overall activation energy for dechlorination of TCE would be higher than the activation energy determined at the reaction rate-limiting step of TCE dechlorination, and even than those of the experimental measurements. This is because at higher surface coverages, bond-breaking reactions that result in more adsorbate-metal bonds in the product state are less favored due to lateral repulsive interactions (33). The surface coverage of TCE in the $\mathrm{p}(4 \times 2)$ supercell $(0.063 \mathrm{ML})$ used in this theoretical study is approximately 4 times higher than that used in the experimental measurements (32). Previous DFT studies of $\mathrm{CO}$ dissociation on $\mathrm{Fe}(100)(34,35)$ and $\mathrm{O}_{2}$ decomposition on $\mathrm{Rh}(111)$ (36) also bear out that higher surface coverage results in a larger calculated activation energy.

The absence of solvation, which is omitted on account of the computational resources required for quantum simulations that include water, may overestimate the activation energy for $\mathrm{C}-\mathrm{Cl}$ cleavage of chlorinated compounds (28). The activation energy is determined as a total energy difference between a transition state and a reactant. Hydration of the partially ionized transition state is more exothermic than hydration of the neutrally-charged reactant, such that dissociation processes are favored in the unhydrated simulations adsorbed chloroethene dechlorination due to the lower activation energies $(28,37,38)$. If solvation effects were included in this study, the calculated activation energies of TCE may be lower than the reported range $(16.6-22.9 \mathrm{~kJ} / \mathrm{mol})$ when these effects are omitted. Considering that DFT calculations may routinely have errors in the range of $19.3 \mathrm{~kJ} / \mathrm{mol}(0.2 \mathrm{eV})(39)$, our results can be used to compare the relative reactivity of the chloroethenes with greater confidence than their absolute reactivities. 


\subsection{Dechlorination of PCE and cis-DCE}

Due to the symmetric geometries of PCE and cis-DCE, the $\mathrm{C}-\mathrm{Cl}$ bonds in these molecules are equally elongated when the species are adsorbed on the iron surface (Table III-2 in Chapter III). Symmetry allows only two possible dechlorination pathways for PCE and only one for cis-DCE according to the dechlorination steps assumed in this study (Figure IV-2). The hypothesized dissociation pathway of cis-DCE is consistent with experimental results that suggest DCE dissociation on $\mathrm{Cu}(100)$ occurs with no carbon deposition and the produces chlorine:acetylene ratio of 2:1 on the metal surface (21). Energy barriers for the first and second $\mathrm{C}-\mathrm{Cl}$ bond cleavages of PCE and cis-DCE are small, less than 11 and $24 \mathrm{~kJ} / \mathrm{mol}$ for PCE and cis-DCE, respectively, and similarly low for TCE dissociation on $\mathrm{PdCu}(110)$ (17). There is a notable difference between PCE and cis-DCE in the relative activation energies for the first and second $\mathrm{C}-\mathrm{Cl}$ cleavages. For PCE, the first chlorine dissociation has a lower activation energy than the second chlorine dissociation, whereas the opposite holds for cis-DCE. The overall dissociation reaction is therefore rate-limited in the second step for PCE and in the first step for cisDCE. For cis-DCE, the second $\mathrm{Cl}$ dissociation has a very low activation energy barrier and should occur promptly after the first $\mathrm{Cl}$ dissociates. This is consistent with the experimental study of cis-DCE dissociation at $185 \mathrm{~K}$ on $\mathrm{Cu}(100)(21)$.

Figure IV-4 and 5 show the energy diagrams for the most favorable dissociation pathways of PCE and cis-DCE on Fe(110), respectively (pathway [1] and [3] for PCE, and pathway [11] and [12] for cis-DCE in Figure IV-2). As in the dechlorination of TCE, the transition states determined from the NEB method for the first and second $\mathrm{Cl}$ dissociation steps of both PCE and cis-DCE more closely resemble the initial structures (reactants) than the final structures (products), suggesting that the dissociation reactions have an early transition state for both species. The dissociation reactions to remove two chlorines from PCE and cis-DCE are strongly exothermic, releasing 514 and $480 \mathrm{~kJ} / \mathrm{mol}$, respectively. Following detachment of the two chlorine atoms from the $\mathrm{C}=\mathrm{C}$ backbone of PCE and cis-DCE, the respective dechlorination product species of dichloroacetylene $(\mathrm{Cl}-\mathrm{C} \equiv \mathrm{C}-\mathrm{Cl})$ and acetylene $(\mathrm{H}-\mathrm{C} \equiv \mathrm{C}-\mathrm{H})$, relocate from the atop adsorption site to the longest bridge site (see Figure IV-4 and 5). Other literature sources report that the most favorable adsorption site for acetylene on $\mathrm{Fe}(110)$ is a 4-fold site, where the $\mathrm{C} \equiv \mathrm{C}$ bond is 
coordinated with four iron atoms (29). This implies that the longest bridge site may be a local minimum closest from the atop site where PCE is adsorbed and the 4-fold site may be a global minimum for dichloroacetylene adsorption on $\mathrm{Fe}(110)$. This also indicates that another activated surface diffusion step would be required for dichloroacetylene to rotate and translate from the atop site to the 4-fold site on $\operatorname{Fe}(110)$. The adsorption configurations of both dichloroacetylene and acetylene are very similar to that of the adsorbed chloroacetylene formed by TCE dechlorination. The $\mathrm{C}-\mathrm{Cl}$ and $\mathrm{C}-\mathrm{H}$ bonds of the adsorbed species bend away from the Fe surface, consistent with a previous theoretical study of acetylene adsorption on $\mathrm{Fe}(100), \mathrm{Fe}(110)$, and $\mathrm{Fe}(111)$ surfaces (29). The calculated geometry of the adsorbed acetylene on the long bridge site of $\mathrm{Fe}(110)$ indicates a $\mathrm{C} \equiv \mathrm{C}$ bond elongation of about $0.2 \AA$, relative to the gas-phase acetylene geometry determined by experiment (30), and a $\mathrm{C}-\mathrm{H}$ bond oriented away from the iron surface at an angle of $58^{\circ}$.

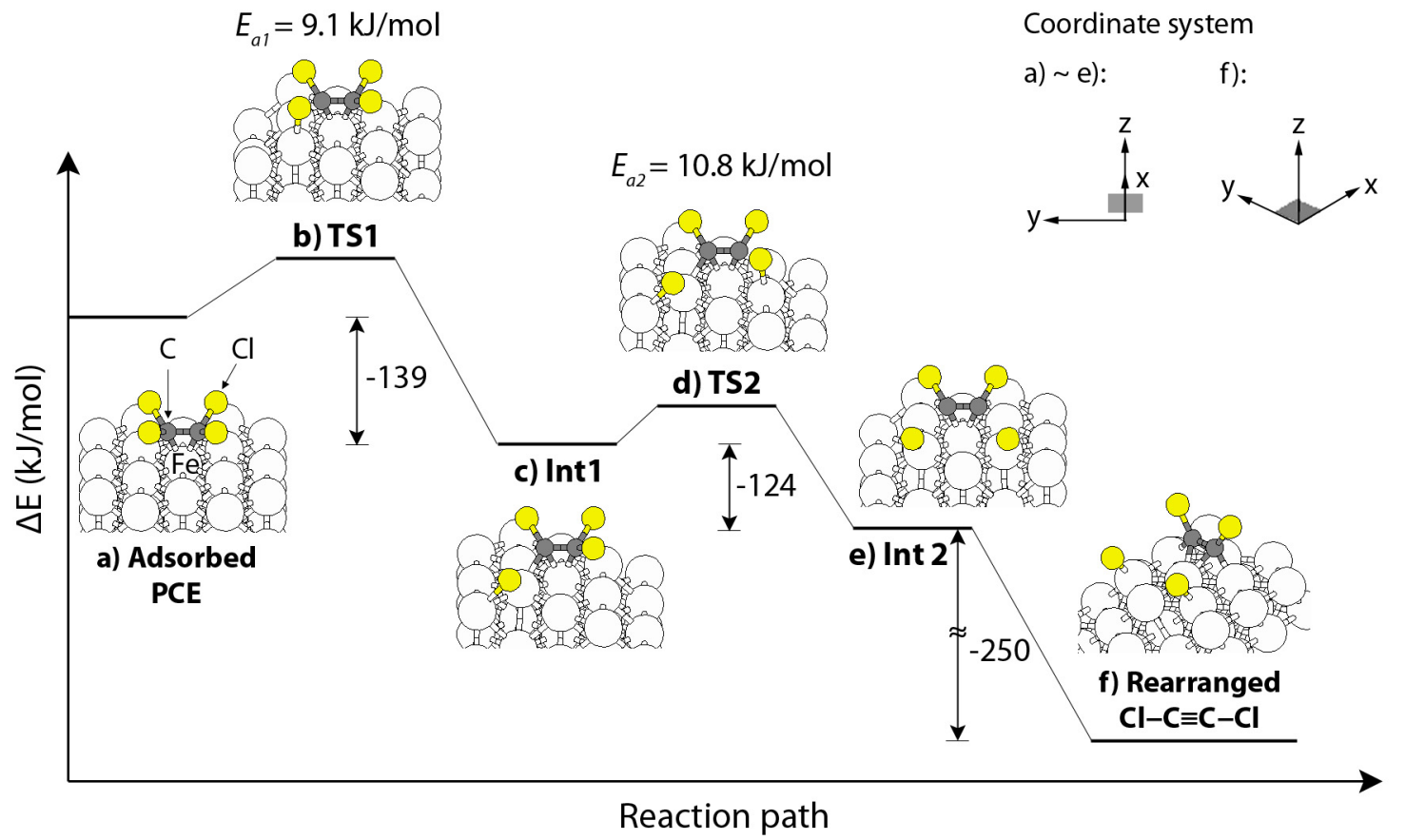

Figure IV-4. Energy diagram for the PCE dechlorination (Pathway [1] and [2] in Figure IV-2). The nomenclature is the same as is used in Figure IV-3. 


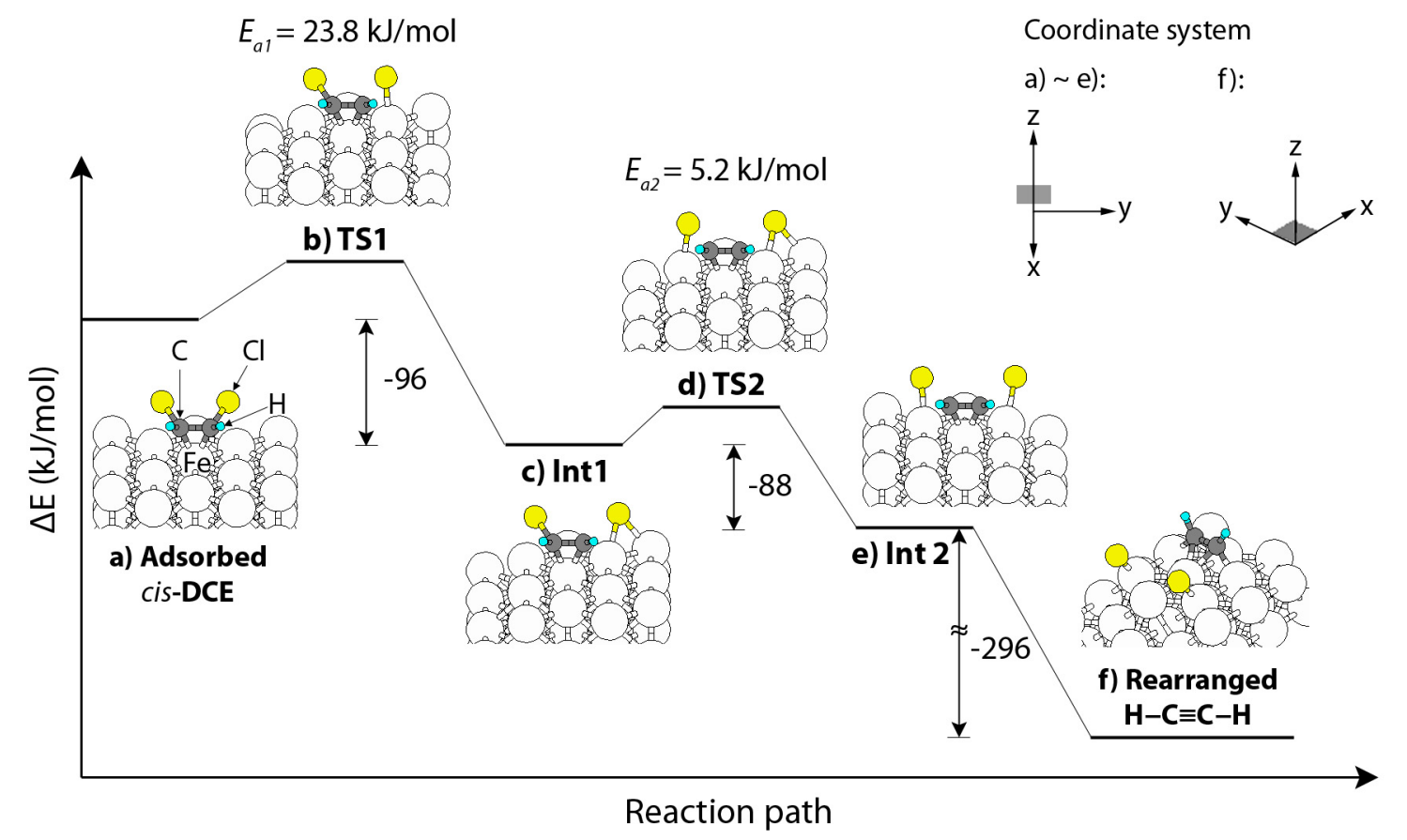

Figure IV-5. Energy diagram for the cis-DCE dechlorination (Pathway [11] and [12] in Figure IV-2). The nomenclature is the same as is used in Figure IV-3.

The second $\mathrm{Cl}$ dissociation of PCE favors Pathway [3] rather than Pathway [2] (Figure IV-2). For the latter pathway, the dichloroacetylene product forms an adsorbed state that is very similar to that of acetylene on Fe(110) (29). However, the dichloroacetylene product from Pathway [3] further dissociates in the geometry optimization, breaking its remaining $\mathrm{C}-\mathrm{Cl}$ bonds and shifting the $\mathrm{C} \equiv \mathrm{C}$ bond at a 4-fold site where it is coordinated with four iron atoms (Figure IV-4 and 5). This full dissociation may be caused by the steric effect of chlorine atoms on the iron surface: although $\mathrm{Fe}(110)$ bridge sites are favored for acetylene adsorption, the proximity of attached chlorines to one another may compel further dissociation to ultimately form acetylene rather than dichloroacetylene in Pathway [3]. Hydrogenolysis of the geometrically optimized $\mathrm{C} \equiv \mathrm{C}$ bond would then yield acetylene at the 4-fold site. Although Pathway [3] is more favorable than Pathway [2] in terms of activation energy, Pathway [2] may be the favored pathway by which dichloroethylene forms through $\beta$ elimination of PCE. Yang et al. (21) detected chlorine and carbon deposition on the $\mathrm{Cu}(100)$ surface after heating TCE to $400 \mathrm{~K}$. The authors noted that TCE produces an 
amount of carbon at least twice as much as the amount of acetylene, and an amount of chlorine six times the acetylene yield, based on the following reaction (21).

$$
2 \mathrm{C}_{2} \mathrm{HCl}_{3} \rightarrow \mathrm{C}_{2} \mathrm{H}_{2}+2 \mathrm{C}+6 \mathrm{Cl}
$$

Hence, at temperatures above room temperature, if hydrogenolysis can occur to the first intermediate formed in the PCE dechlorination pathway (4), Pathway [3] may be more favorable than Pathway [2] because the $\mathrm{C}-\mathrm{Cl}$ bonds will more readily dissociate to release chlorine atoms along this pathway. 


\subsection{Dechlorination rate constants of PCE, TCE, and cis-DCE on $\mathrm{Fe}(110)$}

Vibrational frequencies of gas-phase TCE calculated using the PBE functional with the LanL2DZ double-zeta basis set in Gaussian 03 (20) are compared with experimental data (40) and other theoretical calculations (28). Figure IV-6 and Table IV-1 show the comparison of the vibrational frequencies. The solid line represents the experimental data for the vibrational frequencies of TCE $\left(R^{2}\right.$ correlation factor equal to 1.0). The $R^{2}$ correlation factors of the vibrational frequencies obtained from the Gaussian 03 program, the periodic model used in this study, and Zhang et al.'s theoretical study (28) are 0.998, 0.995, and 0.999, respectively. The calculated results thus agree well with the experimental data.

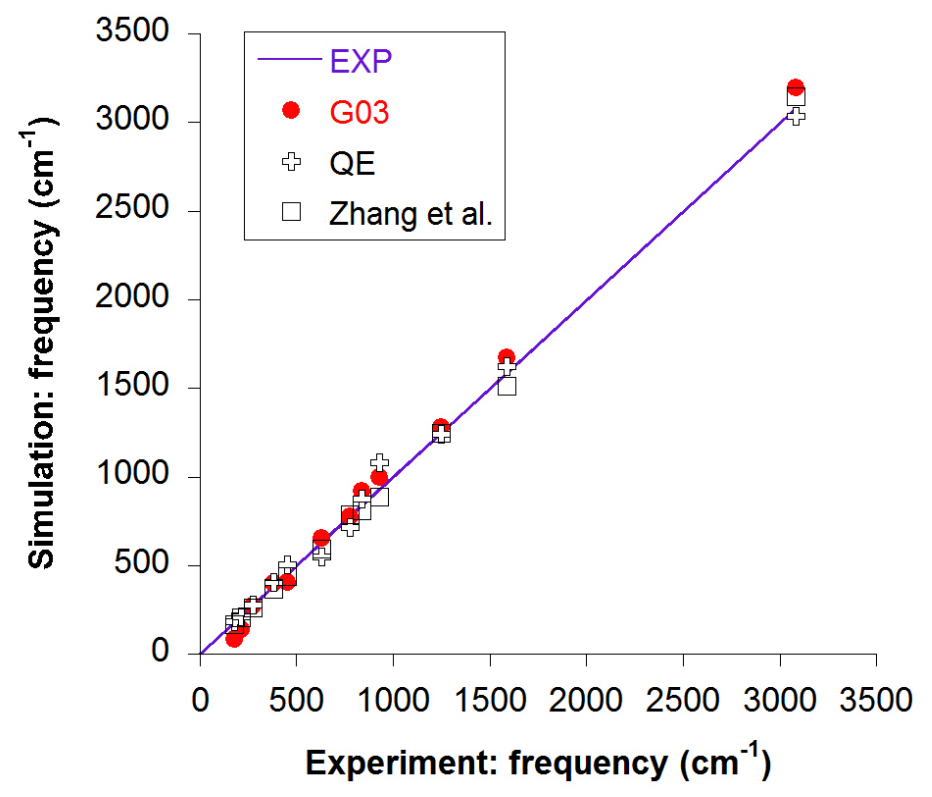

Figure IV-6. Comparison between theoretical and experimental vibrational frequencies (expressed as wavenumbers, $\mathrm{cm}^{-1}$ ) of gas-phase TCE. EXP represents the experimental data (40). G03 and QE are the theoretical calculations obtained from the cluster model (Gaussian 03) and the periodic model (Quantum Espresso). Theoretical values calculated by Zhang et al. (28) are also shown. 
Table IV-1. Comparison of theoretical and experimental vibrational frequencies (expressed as wavenumbers, $\mathrm{cm}^{-1}$ ) for gas-phase TCE. The column titles have the same meaning as the captions listed in Figure IV-6.

\begin{tabular}{cccc} 
EXP & G03 & QE & Zhang et al. \\
\hline 178 & 86 & 183 & 166 \\
215 & 140 & 210 & 204 \\
277 & 272 & 276 & 261 \\
384 & 402 & 406 & 369 \\
451 & 405 & 503 & 437 \\
630 & 652 & 547 & 595 \\
780 & 777 & 717 & 789 \\
840 & 919 & 878 & 811 \\
931 & 1000 & 1079 & 886 \\
1247 & 1283 & 1243 & 1249 \\
1586 & 1671 & 1622 & 1512 \\
3082 & 3198 & 3038 & 3148 \\
\hline
\end{tabular}

Figure IV-7A shows the rate constants for PCE, TCE , and cis-DCE as a function of temperature for the most favored dechlorination pathways shown in Figure IV-2. Figure IV-7B shows the pre-exponential factors ( $A$ factors) of the rate-limiting steps in the dechlorination pathways. As shown in the results, the relative order of reactivity among the chlorothenes is PCE $>$ TCE $>$ cis-DCE. At room temperature $(300 \mathrm{~K})$, the calculated PCE dechlorination rate is 14 times faster than TCE and 338 times faster than cis-DCE. This agrees with experimental studies of chloroethene degradation performed by Johnson et al. (41) and Scherer et al. (42) that reported a dechlorination rate of PCE 5.4 times faster on zerovalent iron than that of TCE. Whereas experimental studies of chloroethene degradation kinetics compare the surface area normalized rate constants $\left(k_{\mathrm{SA}}\right.$ in $\mathrm{L} \mathrm{m}^{-2} \mathrm{~h}^{-}$ $\left.{ }^{1}\right)$, in this study, the degradation rate constants $\left(k_{d e C l}\right.$ in $\left.\sec ^{-1}\right)$ are compared because all theoretical calculations in the study are based on the same Fe(110) surface model. The pre-exponential factors for the rate-limiting step of chloroethene reduction in Figure IV- 
7B do not show any peculiarity in their behavior. However, at temperatures higher than room temperature $(>300 \mathrm{~K})$, the pre-exponential factors for PCE dechlorination are smaller than those for TCE and cis-DCE dechlorination, even though the PCE dechlorination rate constants are higher than those for TCE and cis-DCE. This is because the contribution of the magnitude of the activation energy in the exponential term of the Arrhenius equation far outweighs that of the $A$ factor in the rate constant calculation (Equation (2)). This is also more significant at lower temperatures.
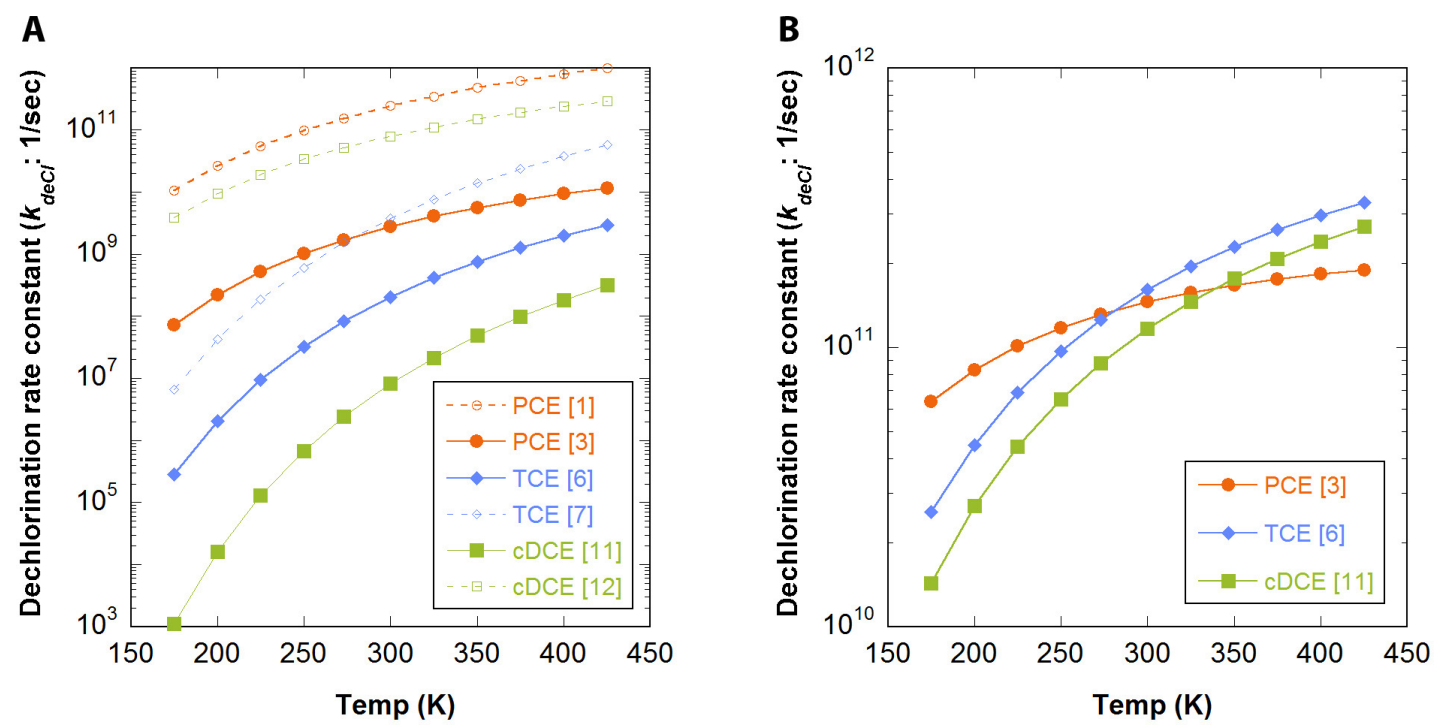

Figure IV-7. Rate constants for the most favored pathways of PCE, TCE, and cis-DCE dechlorination (A) and the pre-exponential factors ( $A$ factors) of the rate-limiting steps (B). The numbers in square brackets denote the reaction pathways labeled in Figure IV-2.

Our theoretical results predicting increased reactivity on zerovalent iron with higher chlorination are in agreement with other experimental results (41-44) in that the dechlorination rate of PCE or TCE is greater than that of cis-DCE. Faster dechlorination with a higher degree of chlorination is also found for chloroethenes on iron-bearing soil minerals such as biotite (45), magnetite (2), and green rust (3). Furthermore, the largest rate constant for TCE dechlorination, followed by PCE and cis-DCE dechlorination, has been reported on zerovalent iron $(43,44)$ and on $\mathrm{Fe}$ (hydr)oxide mimicking chloride green rust (46). The higher degradation rate for TCE over PCE may be due to rapid indirect reduction of TCE by hydrogen $(43,47)$ or competitive sorption $(4,43)$. While 
PCE is reduced primarily via direct electron transfer from iron, TCE is reduced mainly via atomic hydrogen (i.e., hydrogenolysis) (47). Thus, under conditions when hydrogen is present, a higher reaction rate for TCE than PCE might be expected (43, 47-51). In our simulation, we assumed that reduction of chloroethenes occurs solely from direct electron transfer from iron through $\beta$-elimination, with no involvement of surface-adsorbed hydrogen. This may be responsible for the higher reduction rate of PCE than TCE in our simulation results.

Li et al. (52) demonstrated that PCE shows more rapid reduction than TCE on zerovalent iron when the surface is coated with a surfactant to make it more hydrophobic and increase adsorption. Loraine (53) and Alessi and $\mathrm{Li}$ (54) also showed that the rate of PCE reduction increases with longer surfactant treatment time due to increased adsorption of PCE that results. However, surface modification blocks hydrogen release, which leads to the higher reduction of TCE than PCE (52). This also suggests that hydrogen plays an important role when the rate of TCE reduction exceeds that of PCE. A number of studies have shown that the reduction rate of TCE is several times faster than that of PCE $(47,48,51,52,55,56)$. However, these studies did not also measure and compare the dechlorination rates of cis-DCE and vinyl chloride (VC), and so the results of these studies are inconclusive.

The type of iron is also another important factor that affects the relative reactivity of chloroethenes. VanStone et al. (44) presented two different orders in both relative reactivities and carbon isotope fractionations of chloroethenes depending on two different commercial irons. One exhibits the fastest reduction rates on a chloroethene congeners with low chlorination number ( $\mathrm{VC}>c i s-\mathrm{DCE}>\mathrm{TCE}>\mathrm{PCE}$ ); On the other iron, the reactivity ordering TCE $>$ PCE $>$ cis-DCE $>$ VC) is observed. The differences in both the rate constants and the fractionation factors on the different types of iron may be caused by the relative importance of $\beta$-elimination and hydrogenolysis on the iron surface (44). 


\subsection{Electronic property analyses}

To examine dissociated configurations of PCE, TCE, and cis-DCE in detail, the projected density of states (PDOS) are presented for the transition states of the species following the second $\mathrm{Cl}$ dissociation step. Since valence electrons in the outermost orbitals of Fe serve a key role in the adsorption and dissociation of chloroethenes, the PDOS of $d$ orbitals of the Fe surface atoms and $p_{z}$ orbitals (with the $z$ direction perpendicular to the Fe surface) of $\mathrm{Cl}$ atoms were analyzed. Figure IV-8A presents the PDOS of the $d$ orbital of the Fe surface for non-interactive PCE as described in our previous study (Figure III-6 in Chapter III). The sharp and localized peaks of chlorine are attributed to a lack of interaction between the surface Fe atoms and PCE when the latter is far-removed from the Fe surface. Compared to the PDOS of the $d$ orbitals of the clean Fe surface (Figure IV-8A), those with PCE, TCE, and cis-DCE adsorbed on the surface (Figure IV-8B, 8C, and 8D) broaden and shift (i.e., renormalize) to lower energy levels and strongly hybridize with $\mathrm{Cl} 2 p, \mathrm{C} 2 p$, and $\mathrm{H} 1 s$. The broader and strongly modified bands appear -5 to $-15 \mathrm{eV}$ below the Fermi level. This indicates that chloroethenes strongly interact with the $d$ band of the Fe surface atoms in both adsorption and dissociation.

For all PDOS of the transition states (Figure IV-8B, 8C, and 8D), the two $p_{z}$ orbitals of the $\mathrm{Cl}$ atoms show strong hybridization with the $d$ orbitals of the Fe surface atoms. The PDOS of the first chlorine that is dissociated and adsorbed onto the Fe surface (solid red lines: C11 of PCE, Cl3 of TCE, and Cl1 of cis-DCE in Figure IV-8B, 8C, and 8D, respectively) are shifted to a lower energy level than the $\mathrm{Cl}$ atoms still bound to the adsorbate in the transition state (solid blue lines: $\mathrm{Cl} 3$ of PCE, $\mathrm{Cl} 1$ of TCE, and $\mathrm{Cl} 2$ of cisDCE in Figure IV-8B, 8C, and 8D, respectively). This may be attributed to the stable bond formed between the first dissociated chlorine with the iron surface atoms. The $\mathrm{Cl}$ atoms in the transition states are relatively unstable in comparison to those dissociated and adsorbed onto the $\mathrm{Fe}$ surface. More stabilized $\mathrm{Cl}$ atoms are formed through bonding with the $\mathrm{C} \equiv \mathrm{C}$ bond in PCE and TCE $(\mathrm{Cl} 2 \& 4$ of PCE and $\mathrm{Cl} 2$ of TCE in Figure IV-8B and $8 \mathrm{C}$, respectively). 

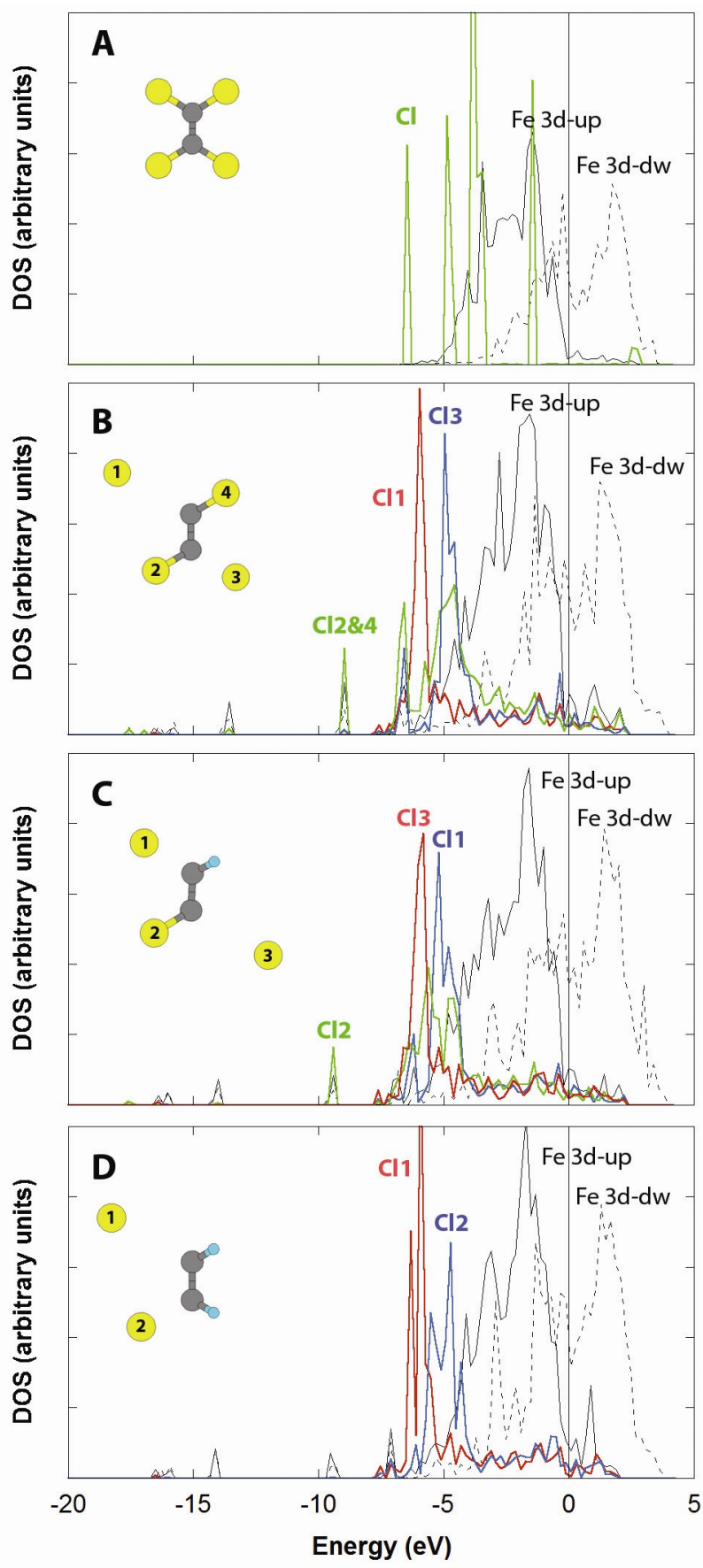

Figure IV-8. Projected density of states (PDOS) for the clean Fe surface with noninteractive PCE (A) and the transition states of PCE (B), TCE (C), and cis-DCE (D). The black solid line and dotted line represent the spin-up and spin-down Fe $3 d$ states, respectively. The red, blue, and green solid lines represent $\mathrm{Cl} 2 p_{z}$ states according to the number marked on the transition state configurations of each adsorbate. Non-bonded Cls that are farthest from the $\mathrm{C} \equiv \mathrm{C}$ bond are the first dissociated and adsorbed $\mathrm{Cls}$ on the $\mathrm{Fe}$ surface. The other non-bonded $\mathrm{Cls}$ are the transition states of the chloroethenes. The Fermi energy is denoted by the vertical line at $0 \mathrm{eV}$. 
In addition to the PDOS analysis, the charge densities of the transition states of the second $\mathrm{Cl}$ dissociated configurations of PCE, TCE, and cis-DCE are presented to assess bonding between atoms. Figure IV-9 shows the top views and side views of the dissociated configurations of the chloroethenes. In all cases, the first chlorine atom dissociated and adsorbed on the Fe surfaces presents little overlap with the carbon atoms in the charge density. The chlorine atoms in transition states, however, still show more overlap in the charge density than the first dissociated chlorine atoms, suggesting the configurations of the transition states more closely resemble the reactants rather than the products. Compared to the adsorbed configurations, the $\mathrm{C}-\mathrm{Cl}$ bonds in the second transition states of PCE, TCE, and cis-DCE are elongated by $0.39,0.46$, and $0.40 \AA$, respectively, which are 23,26 , and $26 \%$ of the elongated distances of the $\mathrm{C}-\mathrm{Cl}$ bonds of the second dissociated configurations of PCE, TCE, and cis-DCE, respectively. Both the elongated $\mathrm{C}-\mathrm{Cl}$ bonds of the transition states and the charge density results support the conclusion that PCE, TCE, and cis-DCE dissociation reactions have an early transition state, as reported in previous studies $(17,28)$.

The side views of the charge densities in Figure IV-9 clearly indicate there is bonding between the dissociated chlorine atoms and the Fe surface. The average distances between three Fe surface atoms at the hollow sites and the dissociated chlorine atom are 2.398, 2.412, and $2.406 \AA$, for PCE, TCE, and cis-DCE, respectively. These $\mathrm{Fe}-\mathrm{Cl}$ bond lengths are close to the length between $\mathrm{Fe}$ atoms and $\mathrm{Cl}$ dissociated from TCE and adsorbed on the $\mathrm{Fe}(100)$ surface $(\sim 2.3 \AA)(28)$ and that of crystalline $\mathrm{FeCl}_{2}$ (2.31 $\AA)(57)$. 

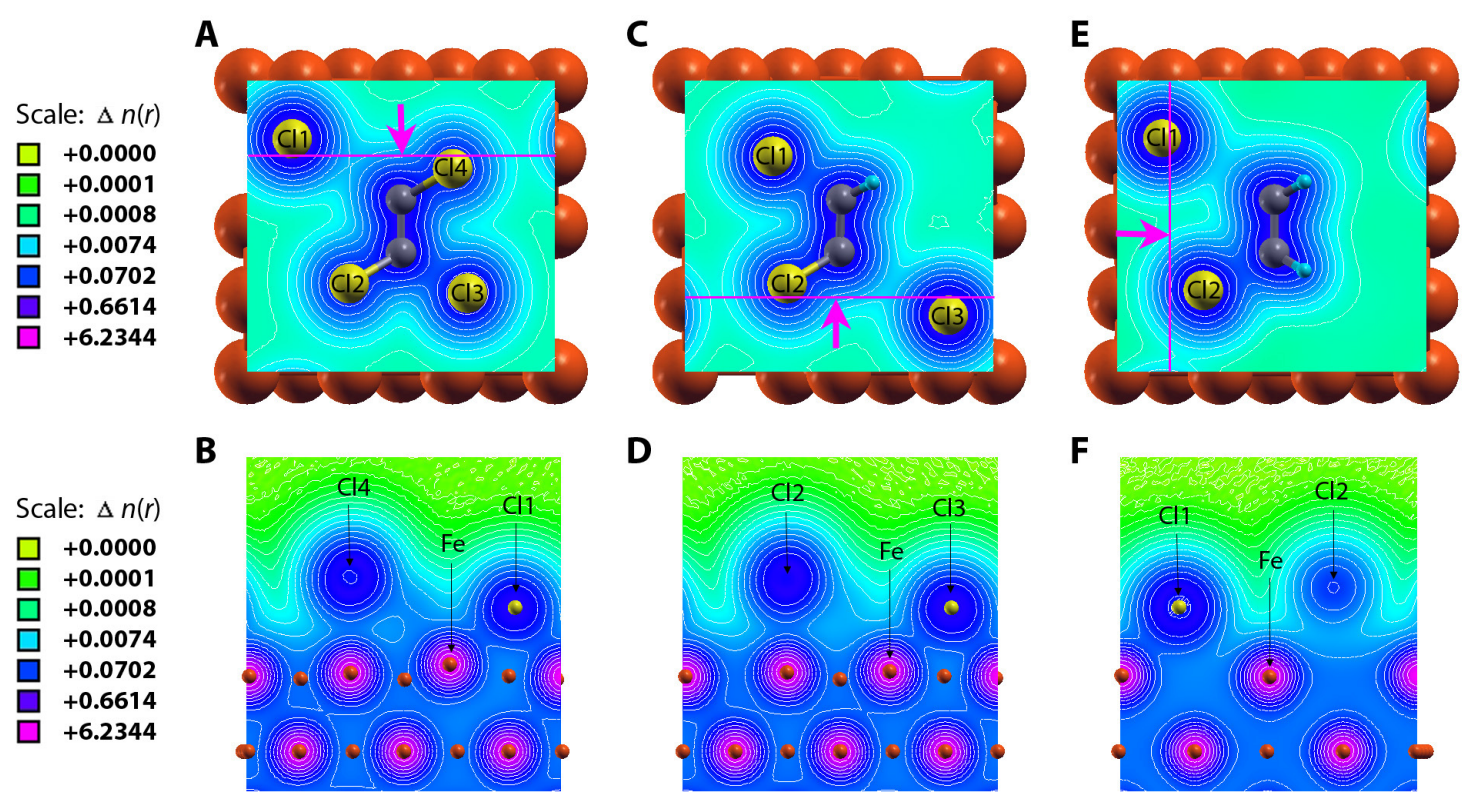

D

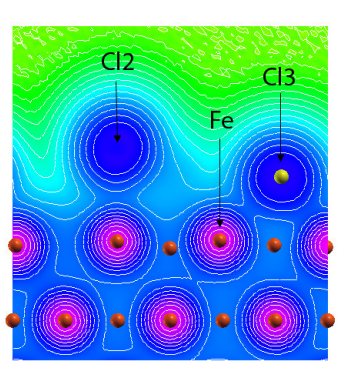

$\mathbf{F}$

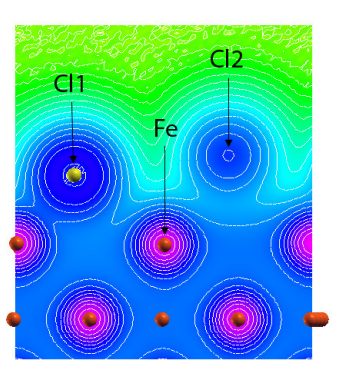

Figure IV-9. Charge densities for the transition states corresponding to the second chlorine dissociation from adsorbed PCE (A \& B), TCE (C \& D), and cis-DCE (E \& F) on the $\mathrm{Fe}(110)$ surface. The first row represents the adsorbed chloroethenes viewed from above. The second row shows the side views perpendicular to the solid lines shown in the first row (arrows indicate the direction of the viewpoint). The numbers shown in the chlorine atoms are the same as those in Figure IV-8. 


\section{References}

(1) Liu, Y. Q.; Majetich, S. A.; Tilton, R. D.; Sholl, D. S.; Lowry, G. V., TCE dechlorination rates, pathways, and efficiency of nanoscale iron particles with different properties. Environ. Sci. Technol. 2005, 39 (5), 1338-1345.

(2) Lee, W.; Batchelor, B., Abiotic reductive dechlorination of chlorinated ethylenes by iron-bearing soil minerals. 1. Pyrite and magnetite. Environ. Sci. Technol. 2002, 36 (23), 5147-5154.

(3) Lee, W.; Batchelor, B., Abiotic, reductive dechlorination of chlorinated ethylenes by iron-bearing soil minerals. 2. Green rust. Environ. Sci. Technol. 2002, 36 (24), 5348-5354.

(4) Arnold, W. A.; Roberts, A. L., Pathways and kinetics of chlorinated ethylene and chlorinated acetylene reaction with $\mathrm{Fe}(\mathrm{O})$ particles. Environ. Sci. Technol. 2000, 34 (9), 1794-1805.

(5) Roberts, A. L.; Totten, L. A.; Arnold, W. A.; Burris, D. R.; Campbell, T. J., Reductive elimination of chlorinated ethylenes by zero valent metals. Environ. Sci. Technol. 1996, 30 (8), 2654-2659.

(6) Hara, J.; Ito, H.; Suto, K.; Inoue, C.; Chida, T., Kinetics of trichloroethene dechlorination with iron powder. Water. Res. 2005, 39 (6), 1165-1173.

(7) Vanderbilt, D., Soft self-consistent pseudopotentials in a generalized eigenvalue formalism. Phys. Rev. B 1990, 41 (11), 7892-7895.

(8) Perdew, J. P.; Burke, K.; Ernzerhof, M., Generalized gradient approximation made simple. Phy. Rev. Lett. 1996, 77 (18), 3865-3868.

(9) Baroni, S.; Dal Corso, A.; de Gironcoli, S.; Giannozzi, P. Quantum ESPRESSO: opEn-Source Package for Research in Electronic Structure, Simulation, and Optimization, v. 3.2; http://www.pwscf.org/: 2007.

(10) Monkhorst, H. J.; Pack, J. D., Special points for brillouin-zone integrations. Phys. Rev. B 1976, 13 (12), 5188-5192.

(11) Jiang, D. E.; Carter, E. A., Adsorption and diffusion energetics of hydrogen atoms on Fe(110) from first principles. Surf. Sci. 2003, 547 (1-2), 85-98.

(12) Jiang, D. E.; Carter, E. A., Adsorption and dissociation of $\mathrm{CO}$ on $\mathrm{Fe}(110)$ from first principles. Surf. Sci. 2004, 570 (3), 167-177.

(13) Henkelman, G.; Uberuaga, B. P.; Jonsson, H., A climbing image nudged elastic band method for finding saddle points and minimum energy paths. J. Chem. Phys. 2000, 113 (22), 9901-9904.

(14) Sheppard, D.; Terrell, R.; Henkelman, G., Optimization methods for finding minimum energy paths. J. Chem. Phys. 2008, 128 (13).

(15) Kokalj, A., Computer graphics and graphical user interfaces as tools in simulations of matter at the atomic scale. Comput. Mater. Sci. 2003, 28 (2), 155-168.

(16) Eichler, A.; Hafner, J., NO reduction by CO on the Pt(100) surface - A density functional theory study. J. Catal. 2001, 204 (1), 118-128.

(17) Barbosa, L.; Sautet, P., Trichloroethene dechlorination reactions on the $\mathrm{PdCu}(110)$ alloy surface: A periodical density functional theory study of the mechanism. $J$. Catal. 2002, 207 (1), 127-138.

(18) van Santen, R. A.; Niemantsverdriet, J. W., Chemical Kinetics and Catalysis (M.V. Twigg and M.S. Spencer, Eds.). Plenum Press: New York and London 1995; p 105. 
(19) Fogler, H. S., Elements of Chemical Reaction Engineering (Fourth Edition). Pearson Education, Inc.: 2006; p 129.

(20) Frisch, M. J., et al. Gaussian 03, Revision C.02; Gaussian, Inc.: Wallingford CT, 2004.

(21) Yang, M. X.; Kash, P. W.; Sun, D. H.; Flynn, G. W.; Bent, B. E.; Holbrook, M. T.; Bare, S. R.; Fischer, D. A.; Gland, J. L., Chemistry of chloroethylenes on $\mathrm{Cu}(100)$ : Bonding and reactions. Surf. Sci. 1997, 380 (2-3), 151-164.

(22) Butler, E. C.; Hayes, K. F., Kinetics of the transformation of halogenated aliphatic compounds by iron sulfide. Environ. Sci. Technol. 2000, 34 (3), 422-429.

(23) Yang, M. X.; Sarkar, S.; Bent, B. E.; Bare, S. R.; Holbrook, M. T., Degradation of multiply-chlorinated hydrocarbons on $\mathrm{Cu}(100)$. Langmuir 1997, 13 (2), 229-242.

(24) Lin, J. L.; Bent, B. E., Carbon Halogen Bond-Dissociation on Copper Surfaces Effect of Alkyl Chain-Length. J. Phys. Chem 1992, 96 (21), 8529-8538.

(25) Lee, C. C.; Doong, R. A., Dechlorination of tetrachloroethylene in aqueous solutions using metal-modified zerovalent silicon. Environ. Sci. Technol. 2008, 42 (13), 4752-4757.

(26) Bernardo, C.; Gomes, J., The adsorption of ethylene on the (110) surfaces of copper, silver and platinum: a DFT study. J. Mol. Struc.-Theochem. 2002, 582, 159-169.

(27) He, Z. H.; Li, Q.; Leung, K. T., Isomeric effects on room-temperature chemisorption and thermal evolution of iso-, cis- and trans-dichloroethylene on Si(111)7x7. Surf. Sci. 2006, 600 (3), 514-526.

(28) Zhang, N. L.; Luo, J.; Blowers, P.; Farrell, J., Understanding trichloroethylene chemisorption to iron surfaces using density functional theory. Environ. Sci. Technol. 2008, 42 (6), 2015-2020.

(29) Anderson, A. B.; Mehandru, S. P., Acetylene Adsorption to Fe(100), Fe(110), and Fe(111) Surfaces - Structures and Reactions. Surf. Sci. 1984, 136 (2-3), 398-418.

(30) Pauling, L., The nature of the chemical bond. Cornell University Press: Ithaca, NY, 1945.

(31) Baranowski, J. M., Bonds in carbon compounds. J. Phys. C Solid State 1986, 19, 4613-4621.

(32) Su, C. M.; Puls, R. W., Kinetics of trichloroethene reduction by zerovalent iron and tin: Pretreatment effect, apparent activation energy, and intermediate products. Environ. Sci. Technol. 1999, 33 (1), 163-168.

(33) Pallassana, V.; Neurock, M.; Lusvardi, V. S.; Lerou, J. J.; Kragten, D. D.; van Santen, R. A., A density functional theory analysis of the reaction pathways and intermediates for ethylene dehydrogenation over $\mathrm{Pd}(111)$. J. Phys. Chem. B 2002, 106 (7), 1656-1669.

(34) Lo, J. M. H.; Ziegler, T., Density functional theory and kinetic studies of methanation on iron surface. J. Phys. Chem. C 2007, 111 (29), 11012-11025.

(35) Bromfield, T. C.; Ferre, D. C.; Niemantsverdriet, J. W., A DFT study of the adsorption and dissociation of $\mathrm{CO}$ on $\mathrm{Fe}(100)$ : Influence of surface coverage on the nature of accessible adsorption states. ChemPhysChem 2005, 6 (2), 254-260.

(36) Inderwildi, O. R.; Lebiedz, D.; Deutschmann, O.; Warnatz, J., Coverage dependence of oxygen decomposition and surface diffusion on rhodium (111): A DFT study. J. Chem. Phys. 2005, 122 (3). 
(37) Zhang, N. L.; Blowers, P.; Farrell, J., Ab initio study of carbon-chlorine bond cleavage in carbon tetrachloride. Environ. Sci. Technol. 2005, 39 (2), 612-617.

(38) Soriano, A.; Silla, E.; Tunon, I., A quantum mechanics-molecular mechanics study of dissociative electron transfer: The methylchloride radical anion in aqueous solution. J. Chem. Phys. 2002, 116 (14), 6102-6110.

(39) Mudiyanselage, K.; Trenary, M.; Meyer, R. J., Kinetics of NH formation and dissociation on $\mathrm{Pt}(111)$. J. Phys. Chem. C 2007, 111 (19), 7127-7136.

(40) Kisiel, Z.; Pszczolkowski, L., Assignment and analysis of the mm-wave rotational spectrum of trichloroethylene: Observation of a new, extended (b)R-band and an overview of high-J, R-type bands. J. Mol. Spectrosc. 1996, 178 (1), 125-137.

(41) Johnson, T. L.; Scherer, M. M.; Tratnyek, P. G., Kinetics of halogenated organic compound degradation by iron metal. Environ. Sci. Technol. 1996, 30 (8), 26342640.

(42) Scherer, M. M.; Balko, B. A.; Gallagher, D. A.; Tratnyek, P. G., Correlation analysis of rate constants for dechlorination by zero-valent iron. Environ. Sci. Technol. 1998, 32 (19), 3026-3033.

(43) Ebert, M.; Kober, R.; Parbs, A.; Plagentz, V.; Schafer, D.; Dahmke, A., Assessing degradation rates of chlorinated ethylenes in column experiments with commercial iron materials used in permeable reactive barriers. Environ. Sci. Technol. 2006, 40 (6), 2004-2010.

(44) VanStone, N. A.; Focht, R. M.; Mabury, S. A.; Lollar, B. S., Effect of iron type on kinetics and carbon isotopic enrichment of chlorinated ethylenes during abiotic reduction on $\mathrm{Fe}(0)$. Ground Water 2004, 42 (2), 268-276.

(45) Lee, W. J.; Batchelor, B., Abiotic reductive dechlorination of chlorinated ethylenes by iron-bearing phyllosilicates. Chemosphere 2004, 56 (10), 999-1009.

(46) Jeong, H. Y.; Kim, H.; Hayes, K. F., Reductive dechlorination pathways of tetrachloroethylene and trichloroethylene and subsequent transformation of their dechlorination products by mackinawite $(\mathrm{FeS})$ in the presence of metals. Environ. Sci. Technol. 2007, 41, 7736-7743.

(47) Wang, J.; Farrell, J., Investigating the role of atomic hydrogen on chloroethene reactions with iron using tafel analysis and electrochemical impedance spectroscopy. Environ. Sci. Technol. 2003, 37 (17), 3891-3896.

(48) Dries, J.; Bastiaens, L.; Springael, D.; Agathos, S. N.; Diels, L., Combined removal of chlorinated ethenes and heavy metals by zerovalent iron in batch and continuous flow column systems. Environ. Sci. Technol. 2005, 39 (21), 8460-8465.

(49) Song, H.; Carraway, E. R., Catalytic hydrodechlorination of chlorinated ethenes bly nanoscale zero-valent iron. Appl. Catal. B-Environ. 2008, 78, 53-60.

(50) Nishijima, W.; Ochi, Y.; Tsai, T. Y.; Nakano, Y.; Okada, M., Catalytic hydrodechlorination of chlorinated ethylenes in organic solvents at room temperature and atmospheric pressure. Appl. Catal. B-Environ. 2004, 51 (2), 135 140.

(51) Farrell, J.; Melitas, N.; Kason, M.; Li, T., Electrochemical and column investigation of iron-mediated reductive dechlorination of trichloroethylene and perchloroethylene. Environ. Sci. Technol. 2000, 34 (12), 2549-2556. 
(52) Li, Z. H.; Willms, C.; Alley, J.; Zhang, P. F.; Bowman, R. S., A shift in pathway of iron-mediated perchloroethylene reduction in the presence of sorbed surfactant - A column study. Water. Res. 2006, 40 (20), 3811-3819.

(53) Loraine, G. A., Effects of alcohols, anionic and nonionic surfactants on the reduction of PCE and TCE by zero-valent iron. Water. Res. 2001, 35 (6), 14531460.

(54) Alessi, D. S.; Li, Z. H., Synergistic effect of cationic surfactants on perchloroethylene degradation by zero-valent iron. Environ. Sci. Technol. 2001, 35 (18), 3713-3717.

(55) Lien, H. L.; Zhang, W. X., Nanoscale iron particles for complete reduction of chlorinated ethenes. Colloid. Surface. A 2001, 191 (1-2), 97-105.

(56) Campbell, T. J.; Burris, D. R.; Roberts, A. L.; Wells, J. R., Trichloroethylene and tetrachloroethylene reduction in a metallic iron-water-vapor batch system. Environ. Toxicol. Chem. 1997, 16 (4), 625-630.

(57) Young, N. A., X-ray absorption fine structure study of the structural changes accompanying spin cross-over in the acetone solvates of [Fe(dppen)(2)X(2)] [dppen=cis-1,2-bis(diphenylphosphino)ethylene; $\mathrm{X}=\mathrm{Cl}$ or $\mathrm{Br}$ ]. J. Chem. Soc., Dalton Trans. 1996, (7), 1275-1281. 


\section{CHAPTER V \\ CONCLUSIONS AND FUTURE WORK}

\section{Conclusions}

This study investigated the relative reactivity of chloroethenes (perchloroethene (PCE), trichloroethene (TCE), and cis-dichloroethene (cis-DCE)) on the Fe(110) surface using density functional theory (DFT) methods. First, iron surface model was constructed in periodic boundary conditions to mimic natural iron surfaces, verifying the physical properties of iron with experimental values. Second, the most thermodynamically favorable adsorption configurations of chloroethenes on the iron surface were determined and the adsorption energies of chloroethenes were calculated. Lastly, the activation energies of chloroethenes along the dechlorination pathways on the iron surface were calculated and then the reaction rate constants at their reaction rate-limit steps were calculated to determine the relative reactivity. Details of electronic properties of the adsorbed and dissociated configurations were also provided in Chapters 3 and 4.

In Chapter 2, the $\mathrm{Fe}(110)$ surface was selected for this study because it is one of mostly commonly observed surfaces and is the most densely packed and thermodynamically stable iron surface. The Fe(110) surface was constructed in periodic boundary conditions after physical properties of bulk iron were verified with experimental values. Each chloroethene molecule was located on the $\mathrm{Fe}(110)-\mathrm{p}(3 \times 2)$ surface slabs at both sides and the slabs were separated in the $z$-direction by a vacuum space of $16 \AA$. This established model structure can be readily applied to future work investigating the effects of solvents, metal impurities, types of iron surfaces, and defects and vacancies of surface atoms on the relative reactivity of chloroethenes on metal surfaces. The optimized simulation parameters can also be readily transferred to other studies investigating interactions between organic pollutants and other metal surfaces.

In Chapter 3, of the adsorption sites examined, an atop site, where the chloroethene $\mathrm{C}=\mathrm{C}$ bond straddles a surface iron atom, was the most energetically favorable site for the 
adsorption of all three chloroethenes. When the effect of solvation is indirectly taken into account in the DFT simulations by considering the hydration energies of chloroethenes in bulk water, the ordering of the adsorption energies of chloroethenes from the aqueous phase onto $\mathrm{Fe}(110)$ is in agreement with experimental observation ( $\mathrm{PCE}>\mathrm{TCE}>$ cisDCE). The adsorption energies of PCE, TCE, and cis-DCE in the aqueous phase are -67.5, -62.6 , and $-54.5 \mathrm{~kJ} / \mathrm{mol}$, respectively. Although there have been the number of experimental investigations showing the adsorption strength trend, as far as we know, this is the first theoretical work demonstrating the relative adsorption strength among the chloroethenes. This chapter also provides the successful use of partial hydration energies of chloroethenes in bulk water on metal surfaces to consider indirect solvent effects in quantum simulations. By examining electronic properties of the adsorbed configuration of chloroethenes, this chapter demonstrated the strong $\pi$ - $d$ orbital hybridization between the chloroethene $\mathrm{C}=\mathrm{C}$ bond and the iron surface, suggesting that adsorbed chloroethenes are strongly activated on $\mathrm{Fe}(110)$ and are likely precursors for subsequent chloroethene dissociation on the Fe surface.

In Chapter 4, the relative reactivity of chloroethenes on the $\mathrm{Fe}(110)$ surface was determined. The relative reactivity order is shown as PCE $>$ TCE $>$ cis-DCE. At room temperature $(300 \mathrm{~K})$, for example, the dechlorination rate of PCE is 14 times and 338 times faster than those of TCE and cis-DCE, respectively. This relative reactivity was determined using activation energies of chloroethenes calculated at their reaction ratelimiting steps. The activation energies of PCE, TCE, and cis-DCE at their rate-limiting steps are $9.9,16.6$, and $23.8 \mathrm{~kJ} / \mathrm{mol}$, respectively. As far as we know, this is also the first theoretical work to suggest the relative reactivity among chloroethenes on iron. However, this theoretical assessment dissociation study was performed assuming a gas phase without considering solvent effects. Since partially ionic transition states are more exothermic in aqueous phase than in the gas phase, not considering solvent effects in this study affects the activation energy by increasing its value as a result of increasing the transition state energy. Further study will be necessary to investigate how the hydration of the partially ionic transition states affects the activation energies of chloroethenes and consequently changes the relative reactivity. 
In summary, this study helps close the knowledge gap in understanding of the interactions and dissociation of chloroethenes on iron. Although the DFT simulations do not account for aqueous phase conditions including $\mathrm{pH}$ or impurities on iron that are common on materials used for groundwater remediation, this study provides better understanding and interpretation of the principal interactions between chloroethenes and iron. From a theoretical perspective, this study will serve as a cornerstone for further studies on the relative reactivities of chlorothenes on zerovalent iron. 


\section{Future Work}

This density functional theory simulations presented here were performed under the gas phase due to limitation of explicit inclusion of the number of water molecules in the quantum simulations. Instead, the water solvent effect was considered in the adsorption energy calculations by correcting for the loss in hydration energy of the chloroethenes in bulk water. It is challenging but important to take into account solvent effects in simulations of metal surface-catalyzed reactions. The thermodynamics and kinetics of surface-catalyzed reactions can be significantly changed under the presence of a solvent medium because partially charged reaction intermediates that are not generally stable in the gas phase can exist in the solution phase and participate in the reaction chemistry. However, explicit inclusion of solvent effects in quantum simulations is almost impossible with current computational technology, especially when relatively large adsorbate molecules are investigated on transition metal surfaces. Although quantum simulations provide detailed understanding of electronic behavior and properties of adsorbates and metal surfaces, another type of molecular simulation tool focusing on molecular behavior, rather than electron behavior would be helpful in understanding of the solvent effects in the adsorption and dissociation of the chloroethenes on the iron surface.

Based on the findings here, future studies need to be conducted in the explicit presence of water molecules using quantum simulations or molecular dynamics (MD) simulations. The first option would still require a huge amount of computational resources. However, if the adsorption and dissociated configurations of chloroethenes and the iron surface optimized in this study are used in the geometry optimizations of chloroethenes under the presence of explicit water molecules, much of computational resources could be reduced. This explicit treatment of water molecules in the quantum simulations would provide molecular level detail of the solute-solvent interactions. This approach would offer an advantage over traditional continuum models that have been used to examine solvent effects by treating the solvent as a continuous medium with a dielectric permittivity that contains a shaped cavity where a solute is located. The other option, MD simulation, significantly reduces computational cost but still provides invaluable help in understanding the behavior of complex systems by employing 
effective empirical potential parameters to describe the metal surface. Recently, ab initio MD has gained widespread acceptance as a tool that adds accuracy and predictive power to MD by using electronic structure calculations to evaluate interaction forces between the atoms.

Another area of investigation that would improve the applicability of this work would be to determine the role of carbon impurities within iron on the relative reactivity of chloroethenes on the iron surface. Carbon impurities may serve as nonreactive sites on the iron surface where chloroethenes may adsorb without further reaction. The adsorbed chloroethenes on the nonreactive sites may thus be misinterpreted as a part of the overall reaction rate. To verify this phenomenon, the same chloroethene-iron surface systems investigated in this study could be modified by replacing several surface or subsurface iron atoms by carbon atoms. The adsorption and dissociation trends among chloroethenes could then be investigated depending on different amounts of carbon atoms or molecules. Besides the carbon impurities, the effects of other metal impurities such as sulfur or nitrogen on the relative reactivity can also be of interest.

Lastly, another interesting study that could be conducted would be to investigate the effect of type of iron surface on the relative reactivity of chloroethenes. This study used $\mathrm{Fe}(110)$ surface to investigate the relative reactivity. Other common surface types of iron are $\mathrm{Fe}(100)$ and $\mathrm{Fe}(111) . \mathrm{Fe}(110)$ is the closest-packed surface of body-centered cubic (bcc) iron with very little relaxation and no reconstruction, while $\mathrm{Fe}(111)$ has a relatively open structure. In addition, defects and vacancies of surface iron atoms would provide interesting insight on the relative reactivity of chloroethenes. The disorder of the surface atoms would affect both adsorption and dissociation of chloroethenes. The effects of defects and vacancies could be readily investigated by removing some or whole rows of surface iron atoms in the constructed model. Different adsorption sites on different iron surface may affect the relative reactivity of chloroethenes. Once the $\mathrm{Fe}(100)$ and $\mathrm{Fe}(111)$ surfaces are constructed and characterized with or without defects and vacancies of surface atoms, the simulation parameters optimized in this study could be used to facilitate the calculations of the adsorption and dissociation of chloroethenes on the other types of iron surfaces. Furthermore, this study could greatly facilitate the same theoretical 
investigations on the relative reactivity of chloroethenes on other mineral surfaces including iron sulfides and iron oxides. 